Departamento de Inteligencia Artificial Escuela Técnica Superior de Ingenieros Informáticos

\author{
PhD Thesis
}

\title{
Knowledge-Graph-Based Semantic Labeling of Tabular Data
}

Author: Ahmad Adel Alobaid

Supervisor: Dr. Oscar Corcho

February 2020 


\begin{abstract}
A lot of data are published on the Web using tabular data formats (e.g., spreadsheets). This is especially the case for the data made available in open data portals by public and private institutions. However, one of the main challenges for their effective (re)use is their generalized lack of semantics: column names are not usually standardized, and their meaning and content are not always clear. In parallel, knowledge graphs have started to be widely adopted by some data providers as a means to publish large amounts of structured data. They commonly use graph-based formats (e.g., RDF) and make references to lightweight ontologies. It is well understood that the reuse of such tabular data may be improved by annotating them with the classes and properties used by the data available in knowledge graphs. Several challenges exist in performing semantic labeling, such as the commonality or duplication of entity names, the difference in measurements and rounding errors of numeric values, and the noise in published tabular data and knowledge graphs. In this work, we present a novel approach to automatically label columns in tabular data with ontology classes and properties referred to by existing knowledge graphs. We evaluated the performance of our approach on entity columns and numeric columns separately. For the entity columns, we applied our approach to annotated tables from the T2D gold standard. For the numeric columns, we manually annotated numeric columns in the T2D gold standard and then applied our technique to this data. We report the performance of our approach using precision, recall, and F1 scores, which is the conventional way to report the performance of semantic labeling in the literature. The experiments showed that our proposed approach successfully labeled the majority of the entity and numeric columns in the used dataset. In contrast with other existing proposals in the state-of-the-art, our approach does not require the use of external linguistic resources, other sources of information, or human in the loop.
\end{abstract}




\section{Acknowledgments}

Many thanks to Professor Oscar Corcho for his support and guidance during the Ph.D. journey. I have learned countless lessons from him. Thanks to my colleague Dr. Juan Sanchez for his insights about the application of statistics. Thanks to Alba Fernández Izquierdo and Dr. María Poveda Villalón for helping me with my presentation at the EKAW conference. Thanks to Dr. Freddy Priyatna, Nandana Mihindukulasooriya, and David Chaves Fraga for all the discussions we had during the coffee breaks. It was a pleasure being part of the Ontology Engineering Group, thanks to all.

I would also like to thank Professor Frank van Harmelen and Professor Stefan Schlobach for hosting me in Vrije Universiteit Amsterdam, where I had the pleasure to work closely with Dr. Wouter Beek. Thanks to Professor Axel Ngonga for hosting me in his group at Universität Paderborn. Thanks to EIT Digital for funding these research stays.

Finally, my sincere gratitude to my parents and family, whom this won't be possible without them. 


\section{Contents}

1 Introduction $\quad 15$

1.1 Definitions . . . . . . . . . . . . . . . 20

1.2 Publications . . . . . . . . . . . . . . . . . 20

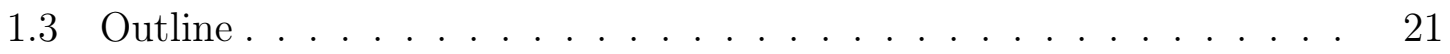

2 Background $\quad 23$

2.1 Knowledge graphs . . . . . . . . . . . . . . . 23

2.2 Tabular Data . . . . . . . . . . . . . . . . 26

2.2.1 Data Formats for Tabular Data . . . . . . . . . . . 27

2.2.2 Data Types . . . . . . . . . . . . . . . . . . . . . 29

2.2.2.1 Types of Numbers . . . . . . . . . . . 30

2.2.3 Associating Data Types to Tabular Data . . . . . . . . . . 33

2.3 Clustering . . . . . . . . . . . . . . . . . . . . . 34

2.3.1 Fuzzy c-means . . . . . . . . . . . . . . 36

2.3.1.1 Initialization . . . . . . . . . . . 37

2.3.1.2 Cluster Center Computation . . . . . . . . 37

2.3.1.3 Membership Assignment . . . . . . . . . . . . 37

2.4 Summary . . . . . . . . . . . . . . . . . . . . . 38

3 State of the Art $\quad 39$

3.1 Techniques. . . . . . . . . . . . . . . . . . . . 39

3.1.1 Geometric Techniques . . . . . . . . . . . . . 40

3.1.2 Probabilistic Techniques . . . . . . . . . . . . . 41 
3.1.3 Logical Techniques . . . . . . . . . . . . . . . . . 45

3.1.4 Non-Machine-Learning Techniques . . . . . . . . . . . . 48

3.2 Data for Evaluation . . . . . . . . . . . . . . . . . . 53

3.3 Limitations . . . . . . . . . . . . . . . . . . . . 55

4 Research Design $\quad 59$

4.1 Research Questions and Hypothesis . . . . . . . . . . . . . 59

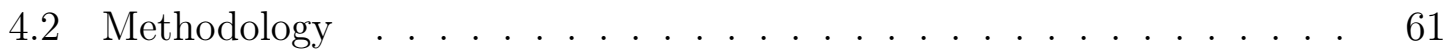

4.3 Data for Evaluation . . . . . . . . . . . . . . . . . . 63

4.3 .1 Olympic Games $2020 \ldots \ldots$. . . . . . . . . . 63

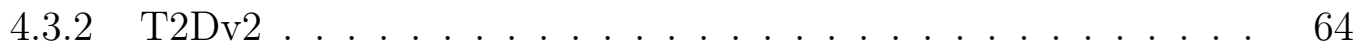

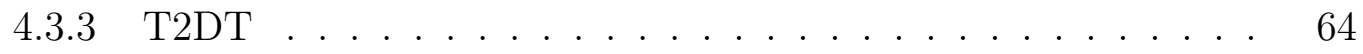

4.4 Assumptions . . . . . . . . . . . . . . . . 65

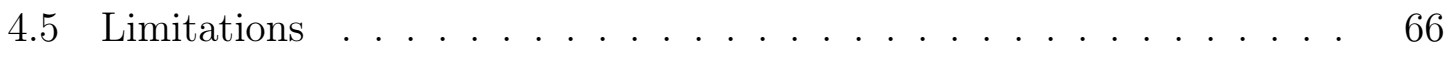

5 Semantic Labeling of Subject Columns $\quad 69$

5.1 An Initial Approach for Semantic Labeling of Subject Columns . . . . 70

5.1 .1 A Working Example . . . . . . . . . . . . . . 70

5.1 .2 Algorithm Description . . . . . . . . . . . . . . 71

$5.1 .2 .1 \quad$ Cells typing . . . . . . . . . . . . 71

5.1.2.2 Class-Graph Construction . . . . . . . . . . 73

5.1 .2 .3 Class Scoring . . . . . . . . . . . . . . . . 74

5.1 .2 .4 Coverage . . . . . . . . . . . . . . 74

$5.1 .2 .5 \quad$ Specificity . . . . . . . . . . 76

5.1 .3 Scoring Functions Proofs . . . . . . . . . . . . . 77

5.1 .3 .1 Coverage . . . . . . . . . . . 78

$5.1 .3 .2 \quad$ Specificity . . . . . . . . . . . . . . 79

$5.1 .3 .3 \quad$ Optimal $\alpha \ldots \ldots \ldots \ldots \ldots \ldots \ldots$

5.1.4 Scoring Functions Construction _. . . . . . . . . 85

5.1 .4 .1 Coverage . . . . . . . . . . . 86

$5.1 .4 .2 \quad$ Specificity . . . . . . . . . . . . 89 
$5.1 .5 \quad$ Experiment Design . . . . . . . . . . . . . . . . 9 90

5.1.6 Experiment Results and Discussion . . . . . . . . . . . . 91

5.2 A Scalable Approach for Semantic Labeling of Subject Columns . . . 94

5.2.1 Subject Column Detection Algorithm . . . . . . . . . . . 95

5.2.1.1 Splitter . . . . . . . . . . . . . . 96

$5.2 .1 .2 \quad$ Spotters . . . . . . . . . . . . . . 97

$5.2 .1 .3 \quad$ Electors . . . . . . . . . . . . . . . . 97

5.2.2 Subject Column Labeling Algorithm _ . . . . . . . . . . 98

$5.2 .2 .1 \quad$ Data splitting . . . . . . . . . . . . . . . 98

$5.2 .2 .2 \quad$ Partition Scoring . . . . . . . . . . . . . . . . . 99

5.2.2.3 Scores Combining. . . . . . . . . . . . . . . 100

5.2.2.4 Column Labeling . . . . . . . . . . . . . . . . 101

5.2 .3 Experiment Design . . . . . . . . . . . . . . . . . . 102

$5.2 .3 .1 \quad \operatorname{Setup} \ldots \ldots \ldots \ldots \ldots \ldots$

5.2.4 Experiment Results and Discussion . . . . . . . . . . . 104

5.2.4.1 Subject Column Detection . . . . . . . . . . . . 104

$5.2 .4 .2 \quad$ Semantic Labeling . . . . . . . . . . . . . 106

5.2.5 Scoring Equivalence to the Initial Approach _ . . . . . . . 108

5.2 .5 .1 Coverage . . . . . . . . . . . . . . 108

$5.2 .5 .2 \quad$ Specificity . . . . . . . . . . . . 110

\section{Semantic Labeling of Numeric Columns 113}

6.1 A Uniform Approach for Semantic Labeling of Numeric Columns . . . 114

6.1.1 Algorithm Description . . . . . . . . . . . . . . . . 114

6.1.1.1 Extraction of Numeric Properties from a Knowledge Graph . . . . . . . . . . . . . . . 114

6.1.1.2 Model Construction . . . . . . . . . . . . . . 117

6.1.1.3 Property Matching . . . . . . . . . . . . . . 118

6.1 .2 Experiment Design . . . . . . . . . . . . . . . . . . 120

6.1 .3 Results and Discussion . . . . . . . . . . . . . . . 121 
6.2 Typology-based Approach for Semantic Labeling of Numeric Columns 124

6.2 .1 Typology of Numeric Columns . . . . . . . . . . . . . . . . 124

6.2.1.1 Example... . . . . . . . . . . . 125

6.2.1.2 Nominal . . . . . . . . . . . . . . . . . . . 125

6.2 .1 .3 Ordinal . . . . . . . . . . . . . . 127

6.2.1.4 Interval and Ratio . . . . . . . . . . . . . . . 127

6.2.2 Algorithm Description . . . . . . . . . . . . . 128

6.2.2.1 Typology Detection . . . . . . . . . . . . . . 129

6.2.2.2 The Detection Order . . . . . . . . . . . . . . 134

6.2.2.3 Model . . . . . . . . . . . . . . . . . . . . . . 134

6.2.2.4 Semantic Labeling . . . . . . . . . . . . . 138

6.2 .3 Experiment Design . . . . . . . . . . . . . . . . . . . . . 139

6.2.4 Experiment Results and Discussion . . . . . . . . . . . 141

$\begin{array}{lll}7 & \text { Conclusion and Future Work } & 147\end{array}$ 


\section{List of Figures}

1-1 An example of a table with ambiguous header . . . . . . . . 16

1-2 An example of manual annotation with OpenRefine and its RDF ex-

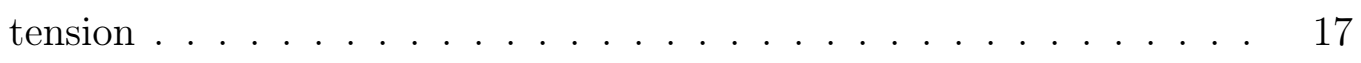

1-3 An example of semantic labeling of a table with a knowledge graph . 18

2-1 The trend of the searches for "Knowledge graph" from Google Trends

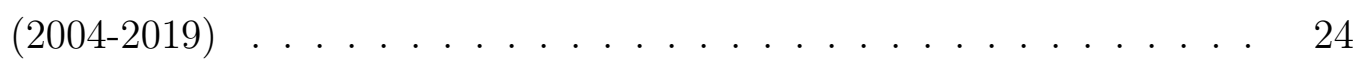

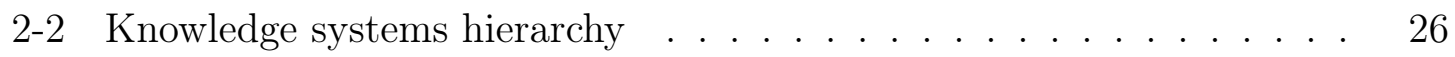

2-3 Trends in tabular data formats . . . . . . . . . . . . . 28

2-4 Trends of searches of terms associated to different data formats . . . 30

$2-5$ The general workflow for pattern clustering [39] $\ldots \ldots \ldots \ldots$

5-1 Example of semantic annotation . . . . . . . . . . . . 72

$5-2$ Semantic annotation workflow . . . . . . . . . . 73

5-3 The coverage score break down for a single cell . . . . . . . . . 88

5-4 Different candidate specificity functions . . . . . . . . . . 90

5-5 Subject Column Detection . . . . . . . . . . . . . 96

$5-6$ Scoring a partition of an entity column . . . . . . . . . . 99

5-7 Combine the class hierarchy of two partitions . . . . . . . . . 101

5-8 Scalable semantic labeling for a single column . . . . . . . . . . 102

5-9 Scalable semantic labeling for multiple tables . . . . . . . . . . . 112

6-1 Example of cluster centers of a model of the class dbo:footballPlayer . 118

6-2 The workflow of typology-based semantic labeling . . . . . . . . 124 
6-3 An example of types and sub-types of numerical columns . . . . . . 126

6-4 An example of hierarchical sub-type . . . . . . . . . . . . . 126

6-5 The detection order . . . . . . . . . . . . . . . . . 135 


\section{List of Tables}

2.1 Different data types discussed in the literature . . . . . . . . . . . 31

2.2 FCM notation and variable names . . . . . . . . . . . . . 36

3.1 Summary of the different approaches with their techniques . . . . . . 54

3.2 Data that are linked or annotated for the different approaches . . . . 56

4.1 Summary of the Olympic Games dataset . . . . . . . . . . . . . . . 64

4.2 Summary of T2Dv2 dataset . . . . . . . . . . . . . 65

4.3 Most common classes in T2Dv2 . . . . . . . . . . . . . . . 65

4.4 Summary of T2DT dataset . . . . . . . . . . . . . . 66

5.1 Notation for scoring functions in Chapter 5 . . . . . . . . . . . 75

5.2 Semantic-labeling scores for Olympic Games with different specificity functions (and the same coverage function) . . . . . . . . . . 92

5.3 Scores of semantic labeling of subject columns in T2Dv2 . . . . . 93

5.4 Subject column detection results on T2Dv2 dataset . . . . . . . . . 105

5.5 Subject column detection with T2DT . . . . . . . . . . 106

5.6 Semantic labeling scores of T2Dv2 . . . . . . . . . . . . 107

6.1 Membership Vector Example . . . . . . . . . . . . . . . . . . . . . . . 119

6.2 Classification scores of the numeric columns of Olympic Games . . . . 121

6.3 Classification scores of the numeric columns of T2Dv2 . . . . . . . . . 122

6.4 Typology Detection Order . . . . . . . . . . . . . . . 135

6.5 Model Features . . . . . . . . . . . . . . . . . 136

6.6 Labeling features per sub-type . . . . . . . . . . . . . . 136 
6.7 Typology Detection and Labeling . . . . . . . . . . . . . . . . 140

6.8 Typology Detection Scores . . . . . . . . . . . . . . . . . . . . . 141

6.9 Typology Labeling Scores _ . . . . . . . . . . . . . . . . . . . . . 142

6.10 Labeling scores of FCM and TTLA approaches applied to T2Dv2 . . 144

6.11 Typology in T2Dv2 dataset . . . . . . . . . . . . . . . . 145 


\section{Chapter 1}

\section{Introduction}

The amount of tabular data available on the Web is enormous. In 2008, Cafarella et al. [14] had already found more than 154 million Web tables. And this number has increased further with the adoption of open data policies by public institutions worldwide. Even though the World Wide Web Consortium (W3C) has published guidelines for data publishing ${ }^{1}$, so as to encourage more data reuse by making datasets published by one entity more easily understood and used by others, most tabular data are being published in CSV and Excel formats, reaching 2 and 3 stars, respectively, in the 5 -star scheme ${ }^{2}$.

Such data are normally published on the Web without annotations. In the case of open data portals, such data may have some general metadata associated with them (e.g., using DCAT). Also, headers are not always present in the tables. Even when they are present, they can be ambiguous. We show an example of such a case in Figure 1-1. Just by looking at this header, we do not necessarily know what the content is about (e.g., it can be the names and heights of buildings, football players, or mountains). This lack of annotations makes it difficult for potential reusers, to understand how the datasets are organized, and what is the meaning of the columns.

There may be many reasons that justify this situation in data publishing, but a generally agreed upon one is the lack of sufficient tools to help data providers publish

\footnotetext{
${ }^{1}$ https://www.w3.org/TR/ld-bp/

${ }^{2}$ http://5stardata.info/en/
} 


\begin{tabular}{|l|l|}
\hline Name & Height \\
\hline manch \\
\hline manch
\end{tabular}

Figure 1-1: An example of a table with ambiguous header

their data in a more understandable and usable manner.

One possibility to overcome this is to create tools or services that generate semantic annotations for such data. The process of assigning semantic annotations to tabular data is referred to as semantic annotation $[36,3]$ and semantic labeling $[52,40]$. The resulting data (once the annotations are exploited) may be made available as virtual or materialized RDF datasets ${ }^{3}$. This is commonly done using mapping languages such as R2RML ${ }^{4}$ [60] and RML ${ }^{5}$ [22]. Such semantic annotations refer to existing ontologies so that data can be described in a formal way that is understandable by humans and machines. Ontologies are used to define the structure of data in knowledge graphs, which acquire and integrate information into ontologies and derive new knowledge [25]. Using ontologies to represent data is a key factor in data interoperability, as heterogeneous data from different sources can be represented using the same terms. We provide the formal definitions of ontologies and knowledge graphs in Chapter 2.

Semantic annotation of tabular data sources is commonly done either manually (e.g., using Open Refine ${ }^{6}$ and its RDF plugin) or semi-automatically (e.g., using Karma $\left.^{7}[41]\right)$. We show an example of manual annotation using Open Refine in Figure 1-2. Manual annotation is a tedious and error-prone process and does not scale. Semi-automatic annotation approaches usually require a lot of prior manual annotations, so that machine learning models are trained so as to learn how to perform semantic annotation. We discuss the shortcomings of the different approaches in more

\footnotetext{
${ }^{3}$ https://www .w3.org/TR/2004/REC-rdf-mt-20040210/

${ }^{4}$ https://www.w3.org/TR/r2rml/

${ }^{5}$ http://rml.io/

${ }^{6}$ http://openrefine.org

${ }^{7}$ http://usc-isi-i2.github.io/karma/
} 


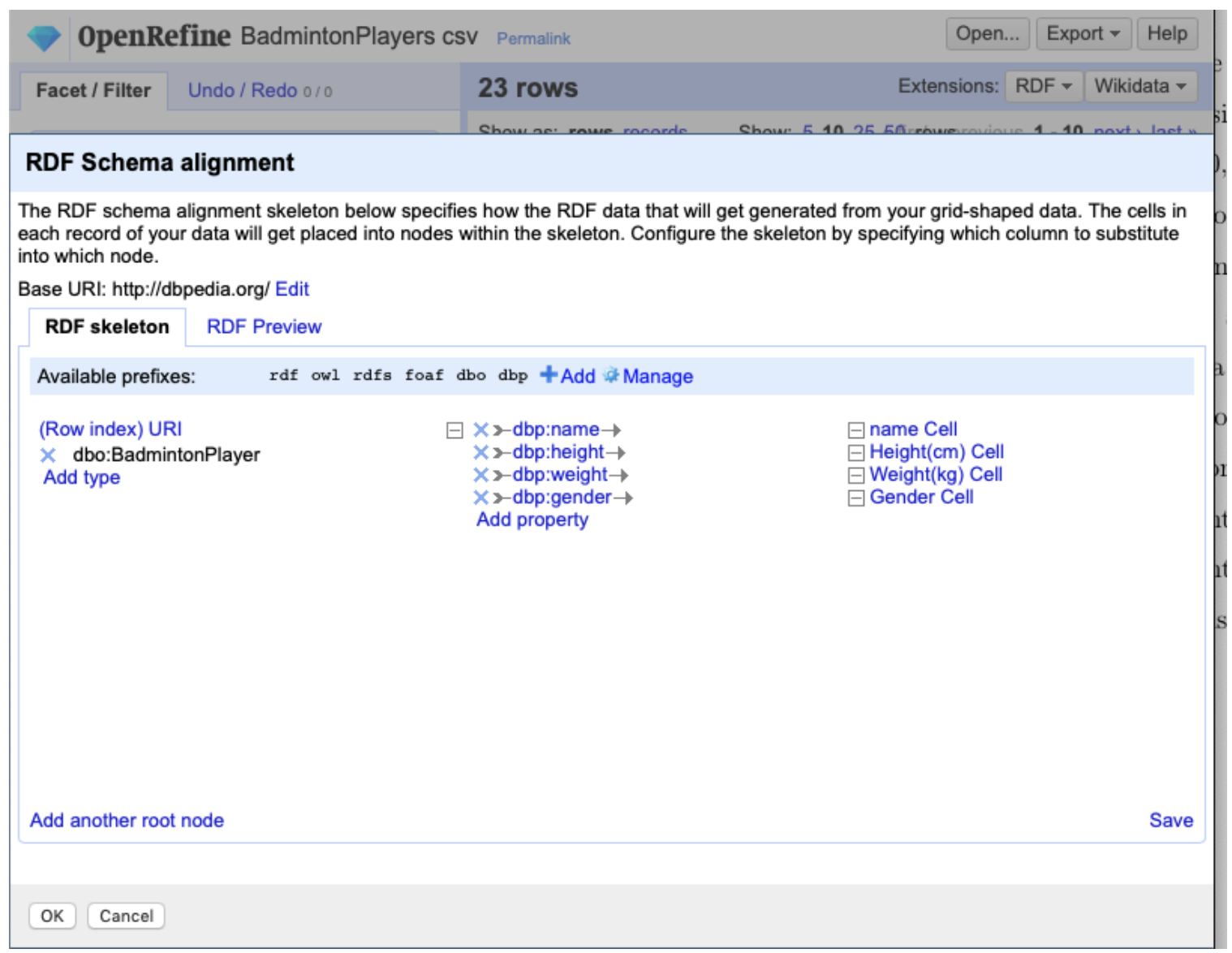

Figure 1-2: An example of manual annotation with OpenRefine and its RDF extension

detail in Chapter 3.

As a proposal to overcome these problems, we hypothesize that we can perform semantic labeling on tabular data automatically using a given knowledge graph as the external source of knowledge. We describe our hypothesis and related research questions in more detail in Chapter 4. In this thesis, we propose a novel approach to match columns in tabular data with their corresponding ontology classes and properties from a knowledge graph. We show an example in Figure 1-3, given a table and a knowledge graph, our approach would annotate the columns using a model that has been trained with the given knowledge graph. We focus on the generally lightweight ontologies that are being used to represent data in knowledge graphs. A key characteristic of our approach is that it does not rely on other commonly used external sources of information (e.g., dictionaries, Wikis) and context around the tab- 


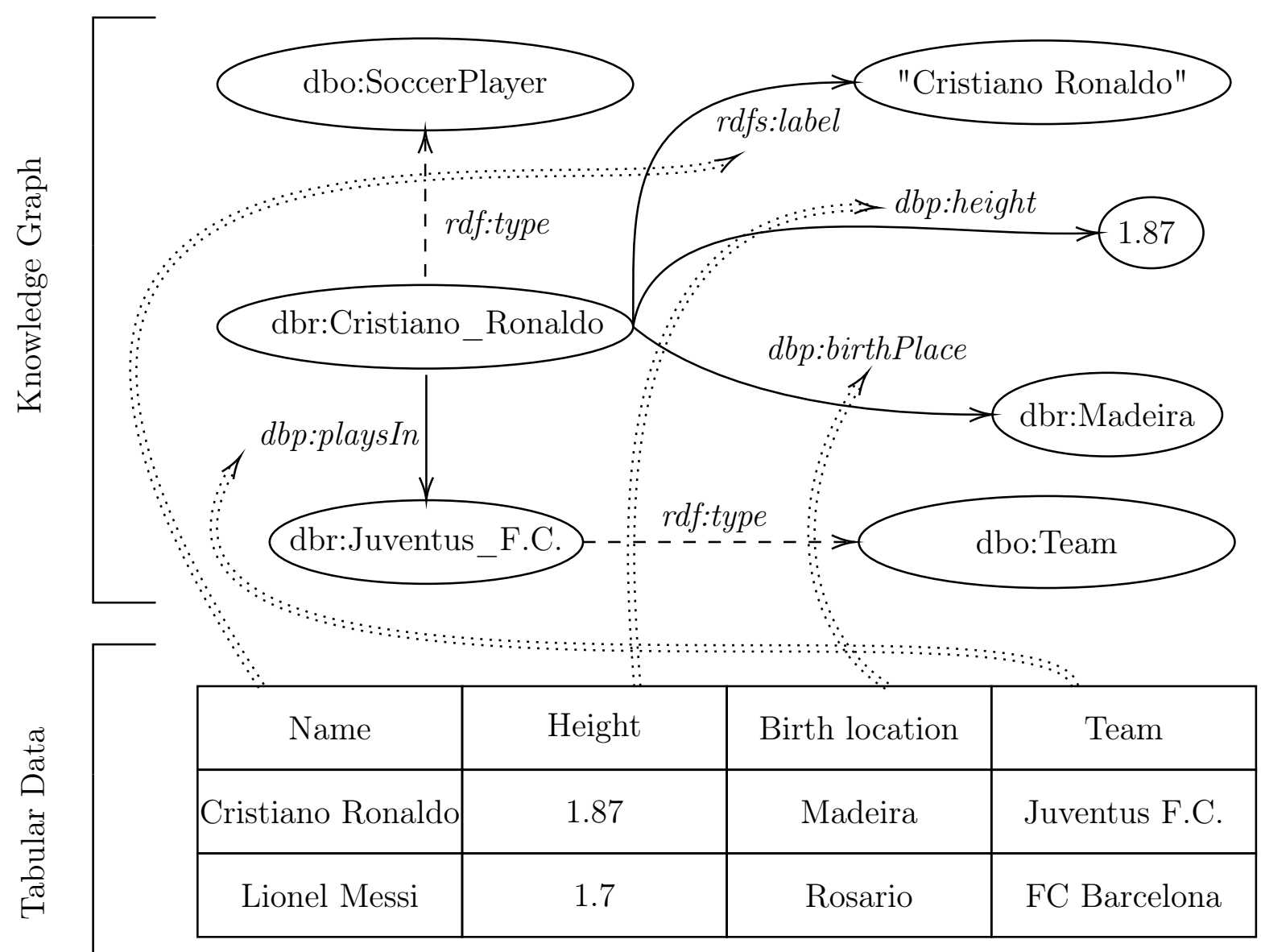

Figure 1-3: An example of semantic labeling of a table with a knowledge graph

ular data(e.g., URL, caption). Moreover, our approach is designed to be automatic so as to overcome the limitations of manual and semi-automatic approaches. The main contributions of our work are:

C.1 A novel approach to label automatically subject columns in tabular data, without considering the context in which the tabular data appears and without relying on other external sources of information beyond a knowledge graph. We follow the intuition that more specific types (e.g., physicist) are more valuable than general types (e.g., person). We formalize our intuition into a set of mathematical formulas (scoring functions), which we present in Section 5.1.

C.2 A scalable approach to label automatically subject columns in tabular data, under the same conditions as before, and without any loss in the labeling scores 
(precision and recall). This is done by splitting large data into smaller pieces, which are processed independently, resulting in intermediate scores. Finally, these intermediate scores are combined to produce the final scores and generate the potential labels. We explain the details of this approach in Section 5.2.

C.3 A semantic labeling approach to match numeric columns with properties from a given knowledge graph without the need to perform entity linking given the subject type. The algorithm detects numeric columns and matches them to numeric properties in the given knowledge graph. This matching is done using fuzzy c-means. We explain the details of how we rely on collections of numbers to build clusters. We present this approach in Section 6.1.

C.4 A semantic labeling approach of numeric columns based on our typology of numeric values. The algorithm detects first the numeric type for each numeric column. Then it applies different functions on the numeric data depending on the detected numeric type. The transformed data are then used to find the equivalent numeric property from a given knowledge graph. The numeric types that we propose, the different data transformations required by the algorithm, and the details of the typology based semantic labeling are described in detail in Section 6.2.

The results of all these approaches are compared against manually annotated tables. Their performance is evaluated using precision, recall, and F1, which is common for semantic labeling approaches. We describe the details of the measurement of the performance of our approach in the evaluation section (Section 4.2) and use it for the evaluation throughout chapters 5 and 6 .

The generated annotations can be further exploited to generate mappings (e.g., R2RML). Such mappings can be used to expose the tabular data source as a virtual (or materialized) knowledge graph that can be easily queried and automatically integrated with other datasets $[60,48,16]$. Now we can answer questions that we may not have been able to answer before. The data may be scattered in multiple files from different sources. Such questions probably involve multiple different datasets, which 
are difficult to navigate, search, and link together. Our approach can automatically add a meaning layer (semantic annotation) for the different files so that they can be automatically linked together. Hence, they can be viewed as a single inter-connected collection of information that can answer questions that we might not have been able to answer before.

\subsection{Definitions}

In this section, we list the definitions of the most important terms that we use in this work.

- Semantic labeling: the processes of assigning classes or properties from one or more ontologies to columns in tabular data. This process is also known as semantic annotation $[76,36,9]$. (see Chapter 5)

- Subject column: the column that contains the subjects of the table $[80,26$, $9,86]$. It contains the labels of the main entity in the table $[80,86,9]$. (see Chapter 5)

- Ontology: "an explicit specification of a conceptualization" [34]. (see Section 2.1)

- Knowledge Graph: "a [system that] acquires and integrates information into an ontology and applies a reasoner to derive new knowledge." [25]. (see Section 2.1)

- Mapping: "the formal tool by which we determine how to link data to the ontology" [57]. (see Chapter 3)

\subsection{Publications}

The following publications have been derived from the work performed in this thesis: 
- Ahmad Alobaid and Oscar Corcho. Fuzzy semantic labeling of semi-structured numerical datasets. In European Knowledge Acquisition Workshop, pages 19-33. Springer, 2018. Alobaid et al. [3]. This publication is related to the semantic labeling of numeric columns in Section 6.1.

- Ahmad Alobaid, Emilia Kacprzak, and Oscar Corcho. Typology-based semantic labeling of numeric tabular data. Semantic Web, 2020. accepted. Alobaid et al. [9]. It includes a typology of numeric columns that we proposed to improve the semantic labeling of numeric columns. It also includes other techniques to label different types of numeric columns. The publication is related to Section 6.2.

- Ahmad Alobaid and Oscar Corcho. Knowledge-graph-based semantic labeling: Balancing coverage and specificity. Semantic Web, 2020. Under review. Alobaid et al. [7]. This article was submitted in the mid of last year and has been under review since then. As the journal follows the open review process, the publication is accessible. This work is related to Section 5.1.

- There is another article about the scalability of subject column labeling. We are waiting for the previous one [7] to be accepted as the work is an extension of it. This part is related to Section 5.2.

\subsection{Outline}

The rest of the thesis is organized as follows. In Chapter 2, we explain the essential topics to understand the rest of the chapters. We briefly talk about the meaning of knowledge graphs in Section 2.1. After that, we present different kinds and formats of tabular data in Section 2.2. Then, we talk about clustering in general and explain more about the fuzzy c-means technique - which is used for labeling of numeric columns - in Section 2.3. In Chapter 3, we talk more about approaches related to semantic labeling, which is the main focus of this work. We talk about the different techniques (Section 3.1), the data used (Section 3.2), and the limitation we observed 
of these techniques (Section 3.3). We specify the research design of our work in Chapter 4. We talk about the methodology we followed, the data we used for the evaluation, and the limitation of our work. Following that, we explain the details of our semantic labeling on subject columns in Chapter 5. It includes the description of the algorithms descriptions, related proofs, the development of the scoring functions, the evaluation of the approach. We explain the semantic labeling approach on numeric columns in Chapter 6. It includes the algorithms description, the proposed typology, related proofs, and the evaluation of the proposed approach. Finally, we conclude our work and propose future work in Chapter 7. 


\section{Chapter 2}

\section{Background}

In this chapter, we shed the light on areas related to our work. The aim is to provide an introductory description on the following areas: knowledge graphs, tabular data, and clustering. Knowledge graph is now a hype, and often used to mean different things, due to the lack of a formal agreed upon definition. Our algorithm uses knowledge graphs to learn how it can annotate given tabular data. After that, we talk about tabular data and the different formats in which tabular data may appear. Finally, we discuss about clustering in general and then in more detail about the fuzzy c-means clustering technique, which we use in this work.

\section{$2.1 \quad$ Knowledge graphs}

Although the term knowledge graph has not made it yet to the most famous dictionaries (e.g., Oxford Dictionary, Cambridge Dictionary, and Merriam-Webster), it has been known for more than two decades. As shown by Ehrlinger and Wöß [25], there are multiple definitions for the term knowledge graph. We discuss them later in this section.

Some people attribute the coining of this term to Google in 2012. However, it has actually existed long before [25]. Google refers to its system as "the Knowledge Graph"

and "Google's Knowledge Graph" [70]. So, when Google announced its "Knowledge Graph", the searches of the term spiked. We aggregated the data to show the results 
for each year using data from Google Trends ${ }^{1}$, which we depict in Figure 2-1. The numbers presented in the figure are relative, as Google Trends only publishes relative numbers (with 100 being the maximum number of hits during the corresponding period).

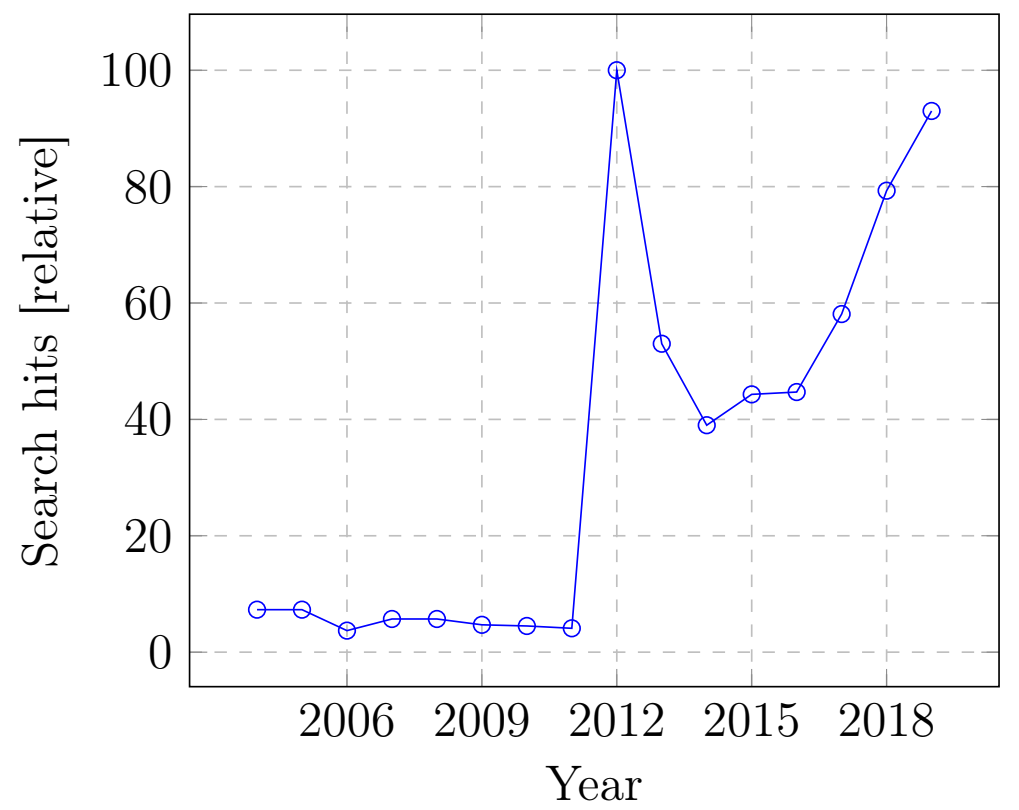

Figure 2-1: The trend of the searches for "Knowledge graph" from Google Trends (2004-2019)

The term knowledge graph also (wrongly) used to refer to a knowledge base or an ontology [25]. Before further discussion of the meaning of the term knowledge graph, we need to explain the meaning for the terms ontology and knowledge base.

The term ontology in computer science has been borrowed from philosophy. Gruber defines it as "an explicit specification of a conceptualization" [34]. Studer et al. define an ontology as "a formal, explicit specification of a shared conceptualisation" [73]. Inspired by early definitions, Feilmayr and Wöß define an ontology as "a formal, explicit specification of a shared conceptualization that is characterized by high semantic expressiveness required for increased complexity." [28]. In simpler terms, an ontology can be thought of as the schema of a database with some preliminary data $[34,25]$.

\footnotetext{
${ }^{1}$ https://trends.google.com/trends/explore?date=all\&q=/m/0jwvf5b
} 
The terms knowledge base and database are sometimes used interchangeably [12], but they are not the same. Wiederhold defines the term database as "a collection of data representing facts" [81]. The main difference is that knowledge bases contain semantics or higher-level abstractions, while databases do not necessarily have semantics; they are more focused on the performance and the representation of factual data $[12,51]$.

In that sense, knowledge graphs should contain more than semantics to be distinguished from knowledge bases. Farber et al. [27] use the term knowledge graph to refer to any $\mathrm{RDF}^{2}$ graph. RDF (Resource Description Framework) is a "standard model for data interchange on the Web." ${ }^{3}$. It is a way to represent information on the Web recommended by the World Wide Web Consortium (W3C). Farber et al. [27] define the term knowledge graph as "an RDF graph consists of a finite set of RDF triples where each RDF triple (s, p, o) is an ordered set of the following RDF terms: a subject $\mathrm{s} \in U \cup B$, a predicate $p \in U$, and an object $o \in U \cup B \cup L$. An $\mathrm{RDF}$ term is either a URI $u \in U$, a blank node $b \in B$, or a literal $l \in L$. U, B, and $\mathrm{L}$ are infinite sets and pairwise disjoint". Others have a broader definition, which can be summarized as a graph of facts, represented as entities of semantic classes and properties with semantic relations between entities $[55,25]^{4}$. Hogan et al. [38] mentioned the similarity to semantic networks [71] but proposed a broader definition, "a graph of data with the intent to compose knowledge". Ehrlinger and Wöß [25] define a knowledge graph as "a [system that] acquires and integrates information into an ontology and applies a reasoner to derive new knowledge." As ontologies have graph structure when defined, this definition implicitly suggests that knowledge graphs have the knowledge in a graph structure, which makes this definition more similar to earlier definitions [55, 25]. The other aspect that is emphasized by Ehrlinger and Wöß [25] is the inclusion of a reasoner, which is practically the main reason for the adoption of knowledge graphs [11, 25].

\footnotetext{
${ }^{2}$ https://www.w3.org/TR/rdf-concepts/

${ }^{3}$ https://www.w3.org/RDF/

${ }^{4}$ Ehrlinger and Wöß [25] reference the definition presented in the Special Issue on Knowledge Graphs of Semantic Web Journal and definition presented in the blog of Semantic Web Company, but the URLs of both are no longer valid so we reference [25] as the secondary source.
} 
In a nutshell, databases represent factual data, knowledge bases include semantics on top, and knowledge graphs include a reasoner to derive extra knowledge (Figure 22). This is the view that we adopt in this work. Nonetheless, different views are still being discussed, and no agreement has been reached yet [38]. Recent discussions took place about the origin and the evolution of the term "knowledge graph" (e.g., Gutiérrez and Sequeda [35] in the International Semantic Web Conference (ISWC) and Hogan et al. [37] in the Dagstuhl Seminar).

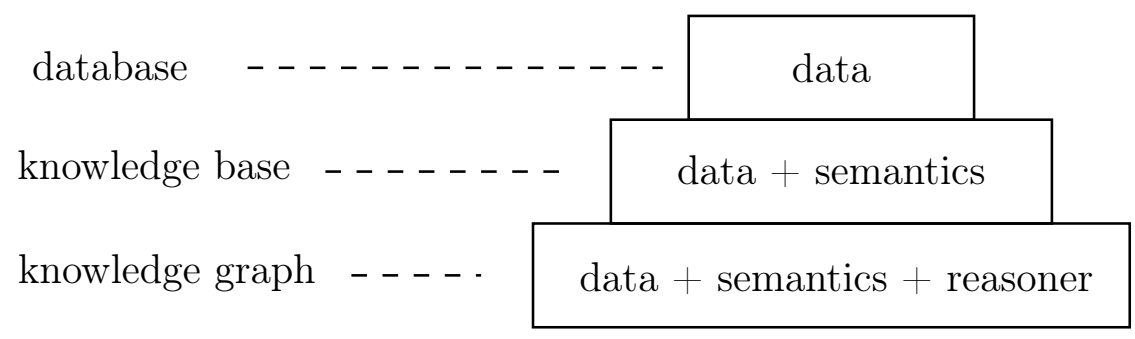

Figure 2-2: Knowledge systems hierarchy

\subsection{Tabular Data}

Tabular data existed long before computers. They used to be published on paper and used to be referred to as "spread sheets", which existed hundreds of years ago [58, 59]. Tabular data are organized data in the form of rows and columns [58, 59]. After the development of mainframe computers, financial calculations used to be performed on those large computers in the 1960s [58]. In 1979, Bricklin and Frankston introduced the first spreadsheet application: VisiCalc [58, 59, 32]. After VisiCalc, there were other applications such as Lotus-1-2-3, Microsoft Excel, StarOffice Calc, and OpenOffice Calc. As the use of the different spreadsheet applications increased, more data are being managed and published in tabular formats. In the next sections, we describe the different formats and data types in which tabular data is usually available. 


\subsubsection{Data Formats for Tabular Data}

In contrast to database systems where data is stored in binary formats (which make them hard to port to other systems), spreadsheets tend to be stored using text-based formats that ease the interoperability of the data (e.g., CSV).

One of the oldest formats to store tabular data is CSV (comma separated values); it has been used since 1972 in Fortran ${ }^{5}$ applications [1]. Even though this format is old, there was no formal specification before 2005 [68]. In Figure 2-3, we show the trend of searches of CSV format in comparison with other formats since $2004^{6}$.

In a CSV file, each line represents a record that is composed of one or more values. The values in a record are separated by a single comma. For the TSV format (tabseparated values), values are separated by a tab character instead of a comma ${ }^{7}$. Both formats dictate that all records should contain the same number of values, and records are separated by a new-line character. One of the main differences between CSV and TSV, besides the use of a different delimiter, is that the CSV format allows the use of its delimiter inside a value while TSV does not. So in CSV files, values can contain commas, and the files can be parsed correctly. This can be achieved by enclosing the value (which contains the comma) by double quotes (see Listing 2.2.1). Double quotes can also be used to enclose values in the absence of a delimiter (comma) in the enclosed value. This may be one of the reasons why the CSV format is more popular than TSV.

Another popular format is XLS, which a binary format used by Microsoft ${ }^{8}$. It is used to store spreadsheet data using their application: Microsoft Excel. In 2008, Microsoft published their Office Open XML format as a standard in ISO ${ }^{9}$. They used XLSX as the extension to Office Open XML format ${ }^{10}$. Durusau and Brauer proposed ODS, a text-based format based on OpenOffice XML. It has been approved

\footnotetext{
${ }^{5}$ https://gcc.gnu.org/wiki/GFortranStandards

${ }^{6}$ the data was aggregated based on Google Trends https://trends.google.com/trends/ explore?date $=a l l \& q=c s v, x l s x$, ods $, x l s, t s v$

${ }^{7}$ https://www.iana.org/assignments/media-types/text/tab-separated-values

${ }^{8}$ https://docs.microsoft.com/en-us/openspecs/office_file_formats/ms-xls

${ }^{9}$ ISO/IEC 29500-1: https://www. iso.org/standard/51463.html

${ }^{10}$ ISO/IEC 29500-3: https : //www. iso.org/standard/51461.html
} 
Listing 2.1: An example of a CSV file with escaped delimiter

Name, Known for

Leonardo da Vinci, "Mona Lisa, The Last Supper"

Michelangelo, David

Salvador Dalí,"The Persistence of Memory"

by OASIS Open Document Format for Office Applications (OpenDocument) [24].

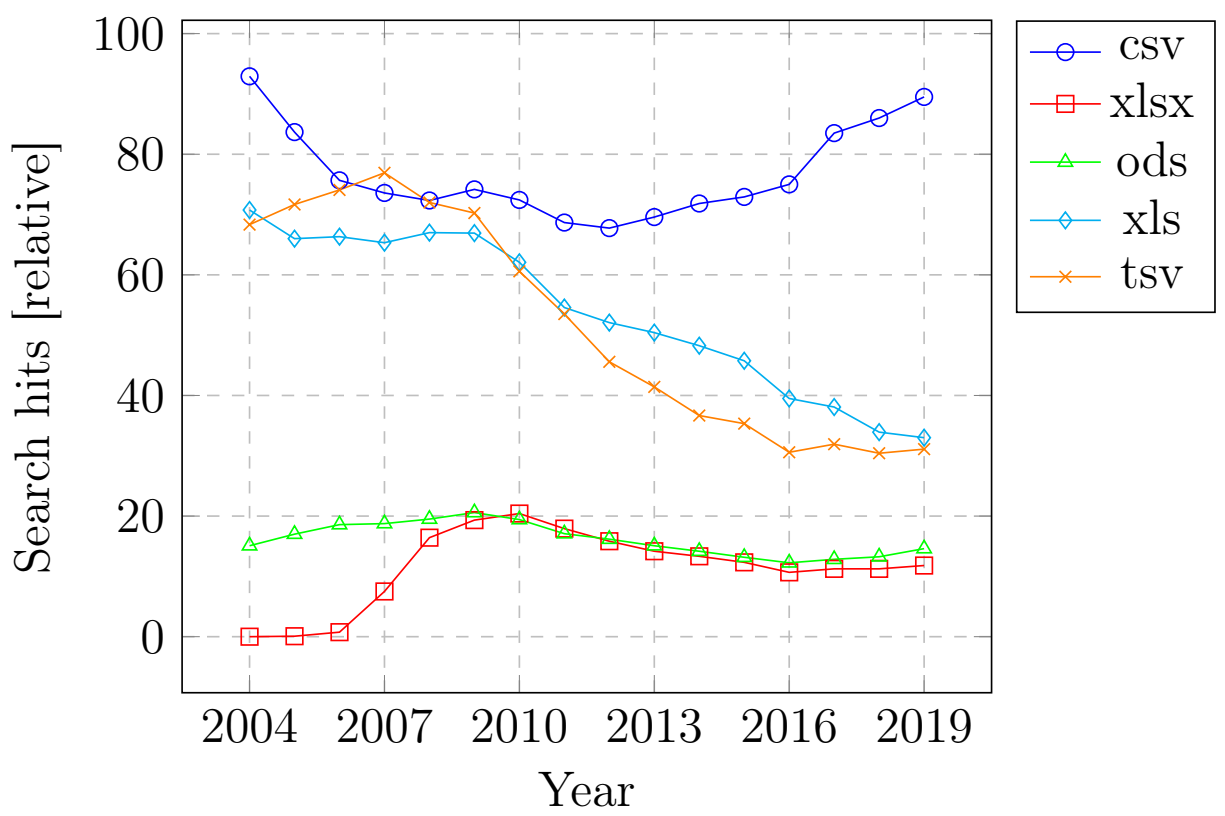

Figure 2-3: Trends in tabular data formats

There are other formats not dedicated for representing spreadsheets, but which are used sometimes to represent spreadsheets with extra information, such as XML and JSON. XML (Extensible Markup Language) was created in 1996 by the W3C XML Working Group with emphasis on ease of use ${ }^{11}$. In contrast to CSV and TSV, XML is composed of "entities" where each "entity" has a "content" and is identified by a name. An "entity" in XML is similar to a record in CSV, but CSV records are flat, while XML can have nested references to "entities" or their attributes. Another competing format to XML sharing a similar goal is JSON (JavaScript Object Notation) [18]. The content of a JSON file is referred to as "JSON text". A "JSON text" should include the "value" of a JSON type, which can be primitive (e.g., string) or structured (e.g.,

\footnotetext{
${ }^{11}$ https : //www . w3.org/TR/1998/REC-xml-19980210
} 
array or object). An "object" is composed of a number of name-value pairs where the values are JSON "values". An "array" is a list of JSON "values" [18]. So, JSON has also a similar tree structure as XML, but more simplistic. XML used to be more dominant in comparison to JSON. But, due to the simplicity of JSON and the speed advantage over XML, JSON became more preferred than XML [53]. We show the trends of searchers for both formats in Figure (2-4). Another format which does not contain a delimiter is fixed-width text file. There is no standard extension for fixedwidth text files, typically ".txt" and ".dat" are used. In a fixed-width text file, each record is terminated by a new line character ${ }^{12}$. Each column has a specific length (number of characters). If the length of a value is less than the width of the column, spaces are inserted at the end, so all values in a column would have the same length. Here we mentioned the most relevant formats, but there are not the only ones. There are also formats used by specific domains such as: Flexible Image Transport System (FITS) which is used by NASA for the archival of astronomical data [33], Common Data Format $(\mathrm{CDF})^{13}$, eXtensible Model Data Format(XMDF) [13], eXtensible Data Model and Format (XDMF) ${ }^{14}$, Hierarchical Data Format(HDF) [43], and netCDF ${ }^{15}$.

\subsubsection{Data Types}

There are different types of values that can be used in tabular data, and there are multiple ways of dividing them. Data in tabular formats can be divided into two main types: strings and numbers $[84,62,56,3]$. They might be divided further into three or more types $[75,80,61,64,66,86,54]$. We list the different data types reported in the literature in Table 2.1.

We can see that some do not distinguish the different kinds of numeric data $[80$, $84,62,64,66,56,86,3]$ while some others do [75, 61, 50, 54].

The reported difference in numeric types is whether the numbers are nominal (e.g.,

\footnotetext{
${ }^{12}$ https://www.oracle.com/webfolder/technetwork/data-quality/edqhelp/Content/ introduction/getting_started/configuring_fixed_width_text_file_formats.htm

${ }^{13}$ https://cdf.gsfc.nasa.gov/

${ }^{14}$ http: //www.xdmf .org/index.php/Main_Page

${ }^{15}$ https : //www . unidata.ucar.edu/software/netcdf/docs/faq.html
} 


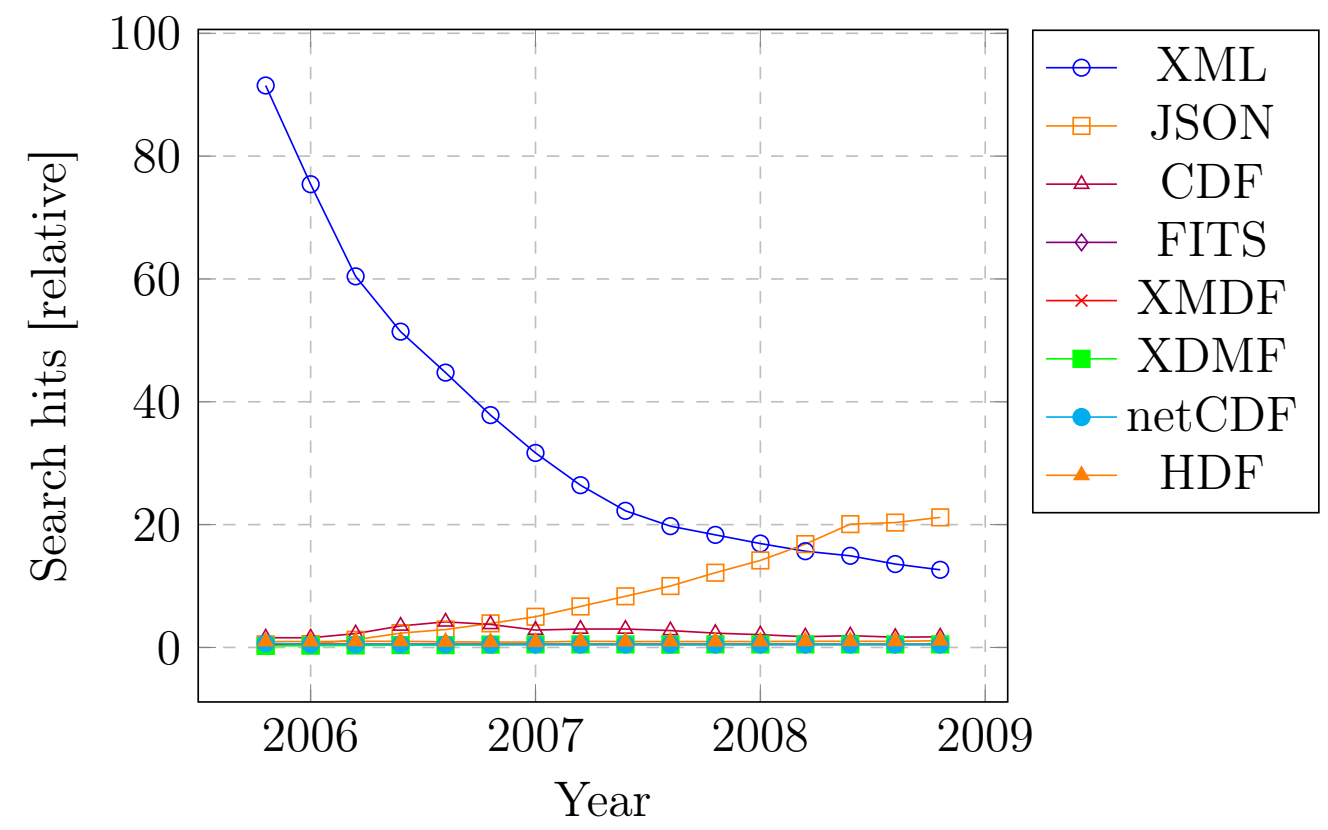

Figure 2-4: Trends of searches of terms associated to different data formats

numbers printed on the t-shirts of football players) or measurements (e.g., weights of football players). We explain the details of the different types of numbers next.

\subsubsection{Types of Numbers}

Mosteller and Tukey said: "Just writing in digits does not make a number"[49]. On the other hand, Stevens and Birkhoff [72] typology of numbers does not quite agree with this ${ }^{16}$. They propose four types of numbers: nominal, ordinal, interval, and ratio.

In Nominals, digits are just like names, so they do not imply extra meanings like order. For example, if we have two names, John and Adam, we cannot say that the name John is greater (or less) than the name Adam. An example of nominals are the digits printed on the shirts of football players: they only distinguish the players, but it doesn't have a "greater than" or "smaller than" relationship associated to it. Therefore, the only operation available is to compare two nominals, whether they are

\footnotetext{
${ }^{16}$ The paper was written by Stevens, but Birkhoff helped to complete the list of types. They refer to nominals as numbers, but they (along with Mosteller and Tukey) agree that numbers (or collection of digits) can carry different meaning, and hence the different types. Stevens approach the typology from the measurements points of view while Mosteller and Tukey view is more from a data analysis point of view.
} 


\begin{tabular}{ll}
\hline Work & Data types \\
\hline Syed et al. [75] & $\begin{array}{l}\text { numbers, dates, addresses, stock, ticker symbols, ISBN num- } \\
\text { bers }\end{array}$ \\
Venetis et al. [80] & number, date, text \\
Zhang [84] & number, string \\
Quercini et al. [61] & $\begin{array}{l}\text { phone numbers, URLs, email addresses, numeric values, geo- } \\
\text { graphic coordinates, long values, location, date, entity names }\end{array}$ \\
Mulwad et al. [50] & string mentions, numbers and measurements \\
Ramnandan et al. [62] & numeric data, textual data \\
Ritze et al. [64] & string, numeric value, timestamp, coordinate \\
Pham et al. [56] & numeric data, textual data \\
Ritze et al. [66] & string, numeric, date, reference \\
Zhang [86] & named entity, number, date expression, long text, other \\
Alobaid el at. [3] & numeric data, textual data \\
Oulabi et al. [54] & text, nominal string, instance reference, date, quantity, nom- \\
& inal integer
\end{tabular}

Table 2.1: Different data types discussed in the literature

the same or not.

Ordinal type implies an order among a set of elements. So one appears before the other. There is the concept of greater than (or less than) between two elements. An example would be ordering the dishes in a menu from the most favorable to the least favorable, or differences in responses in satisfaction surveys between "very satisfied" and "somewhat satisfied" [44]. However, there is no regard to the difference between the two items. For example, if a person likes dark chocolate more than white chocolate (darkchocolate $>$ whitechocolate), there is nothing about the difference between his likeness of dark chocolate and white chocolate. Such an operation could be referred to as "illegal" [72].

The Interval scale is used to denote the increase or expansion in some way on a scale. A classical example presented by [72] is the use of two different scales to measure the temperature. We (humans) use Centigrade and Fahrenheit, and we have 
a way to transform the temperature from one scale to the other. The intervals are the same (e.g., the difference between 10 and 20 degrees Celsius is the same as the difference between 25 and 35 degrees Celsius) [44].

Ratio scales refer to things we measure that contain a real zero such as counts, fundamental, and derived ratios. Counts represent the number of elements or occurrences (e.g., number of eggs in a basket). Fundamental ratio represents measurements taken directly (e.g., when the width of the table is taken directly using a measuring tape or a ruler). Derived ratio is the mathematical function of the magnitudes of a simple (fundamental) ratio measure (e.g., computing the speed of a car using a measured time and distance). The difference between ratio and interval is the existence of a true zero ${ }^{17}$.

For example, when we talk about temperature, there can be an agreement on a zero value (for example 0 in Celsius and 32 in Fahrenheit for the freezing point of water at 1 atmosphere unit), but it is not a true zero point ${ }^{18}$ (it does not mean a complete absence of temperature). Such a point is referred to as the "starting" point $[49,44]$.

Some measures are not considered by Stevens, such as the cyclical measures [17]. An example of such cyclical measure is the angle; Chrisman said: "the direction $359^{\circ}$ is as far from $0^{\circ}$ as $1^{\circ}$ is" [17]. The added levels presented by Chrisman are: Log-interval, extensive ratio, cyclic ratio, derived ratio, counts, and absolute ${ }^{19}$. Loginterval is similar to the interval scale with the scaling happening on the exponent level. Extensive ratio is the same as the fundamental ratio explained by Stevens (e.g., the width of a book). Cyclic ratio is the same as the extensive ratio where it is bounded and repeat. Absolute is when a scale is predetermined and cannot be transformed while preserving the meaning.

Mosteller and Tukey have a similar categorization: names (nominal), grades and ranks $^{20}$ (ordinal), amounts (fundamental ratio), balances ${ }^{21}$ (derived ratio), counts,

\footnotetext{
17 also referred to as "real" and "absolute".

${ }^{18}$ Note that we are not talking about Kelvin scale, where zero is never reached [42]

${ }^{19}$ Chrisman suggestion is to consider them as separate types

${ }^{20}$ ranks are numbered while grades are labels (e.g., A, B, ...)

${ }^{21}$ It is similar to log-interval but also is a ratio, so we consider it here a derived ratio
} 
counted fractions $^{22}$ (absolute). However, it lacks the following: interval ${ }^{23}$, log-interval, and cyclical ratio.

Types of numbers are also referred to as kinds of numbers, scales of measurement, and levels of measurement [72, 49, 17, 44].

\subsubsection{Associating Data Types to Tabular Data}

Different formats exist to represent data, some of them include embedded schemata (e.g., CDF, netCDF) while others do not (e.g., XML, JSON, CSV). Due to the spread of formats such as XML, JSON, and CSV, and the need for an easy way to describe the data and validate the types and structure of the data, several ways were proposed. In 2000, the first draft of XML Schema was published ${ }^{24}$. It is a way to define the schema that an XML data file should follow. XML Schema follows the same format as XML. There is also a schema for JSON called JSON Schema ${ }^{25}$. Both XML and JSON schemata support simple types (e.g., string) and complex types (which can be composed of simple and complex types too). Such schemata are useful for data description and validation. They are used to describe the types and structure of the data. They can also be used to ensure that the structure of the data matches the expected schema. There are other ways that originated from the semantic web community to describe data using the concepts of Linked Data ${ }^{26}$ - where data are linked together and described using shared concepts. JSON-LD - which stands for JSON for Linked Data - is one way to describe data. The data in JSON-LD are described in terms of ontologies. JSON-LD is also exploited by search engines to interpret data published on the Web ${ }^{27}$. Another way to describe data is CSVW, which stands for CSV on the Web. It is a way to describe one or more CSV files using ontologies. It also allows specifying data types and validating the published

\footnotetext{
${ }^{22}$ It is not exactly equal as absolute; it is more general than counted fractions, but it is enough approximation for our purpose in this paper.

${ }^{23}$ Even though Mosteller and Tukey warns the reader when re-expressing zeros, they do not create a separate category for it

${ }^{24}$ https : //www.w3.org/TR/2000/WD-xmlschema-0-20000225/

${ }^{25}$ https: //json-schema.org/draft/2019-09/json-schema-core.html

${ }^{26}$ https://www.w3.org/DesignIssues/LinkedData.html

${ }^{27}$ https ://schema.org/docs/datamodel.html
} 
data.

\subsection{Clustering}

Clustering is a descriptive knowledge discovery technique that consists in gathering data points (also known as patterns [39]) into one or more clusters (or groups) ${ }^{28}$. Clustering techniques, in general, are composed of multiple steps. We show the general workflow presented by [39] in Figure 2-5. This figure contains the first three steps, as the fourth and fifth steps can be more subjective and not needed in all cases. We describe the five clustering steps (as presented by Jain et al. [39]):

1. Pattern representation: it refers to the numeric information about the pattern, which is crucial depending on the technique used (e.g., number of clusters, number of patterns, scale, ...). This step also includes feature extraction and selection. Jain et al. [39] defined them as follows: "Feature selection is the process of identifying the most effective subset of the original features to use in clustering" and "feature extraction is the use of one or more transformations of the input features to produce new salient features."

2. Pattern proximity measure: refers to the function used to compute the distance between patterns (e.g., Euclidean distance).

3. Clustering: grouping of patterns (or groups), which can be hard (each pattern belongs to a single cluster) or fuzzy (a pattern can belong to multiple clusters).

4. Data abstraction: refers to the description of each cluster into a simple and compact format.

5. Output assessment: is the evaluation of the performance or quality of the results. This can be subjective and often depends on the domain.

There are several clustering techniques in the literature $[39,67]$. Here we present different aspects of clustering algorithms as presented by Jain et al. [39]:

\footnotetext{
${ }^{28}$ https://www . dictionary $\cdot$ com/browse/clustering?s=t
} 


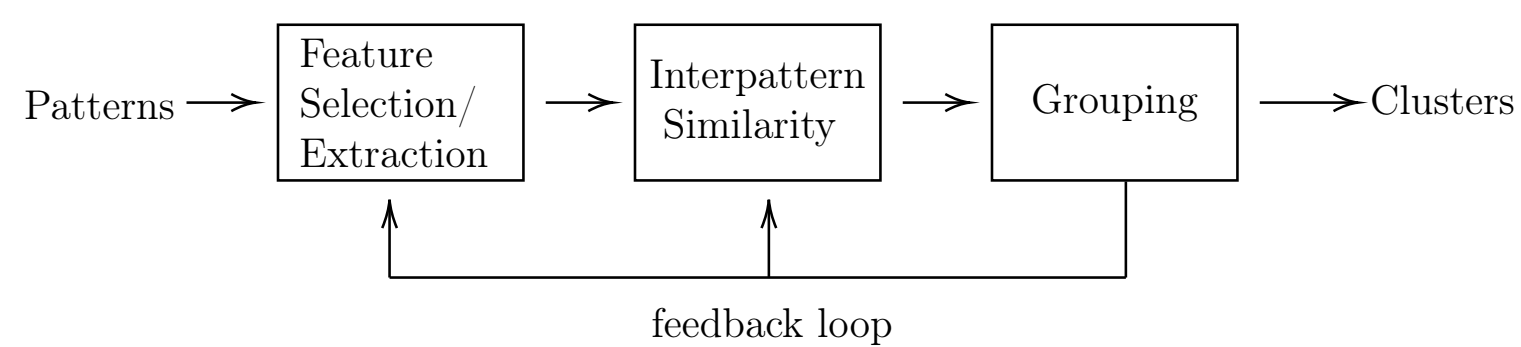

Figure 2-5: The general workflow for pattern clustering [39]

- Divisive vs. agglomerative: the former start with a single cluster and then divide clusters into smaller clusters, while the later start with a lot of small clusters, and then smaller clusters merge until a threshold is met, resulting in a smaller number of clusters of larger size.

- Hard vs. fuzzy: hard clustering techniques assign each data point or pattern to a cluster. In fuzzy clustering, a data point can belong to multiple clusters with a percentage.

- Polythetic vs. monothetic: polythetic techniques use all the features at the same time. Monothetic techniques use one feature at a time, and then divide the cluster using the second feature and so on.

- Stochastic vs deterministic: some approaches optimize (minimize) the total error via random search (stochastic) while other techniques follow deterministic rules.

- Incremental vs. non-incremental: in the early days of clustering algorithms, restrictions (e.g., data size) was not taken into account. Later on, techniques were developed to take into account such restrictions (e.g., limit the number of scans of the data or the number of considered patterns).

In the following section, we will expand more on the fuzzy c-means technique, the one used in the approach that will be presented in Chapter 6 . 


\subsubsection{Fuzzy c-means}

Fuzzy c-means (FCM) is a generalization of the k-means clustering algorithm [10]. FCM was introduced by Bezdek et al. [10], based on the fuzzy sets introduced by Zadeh [83]. In k-means, a point can belong to only one cluster, while in fuzzy c-means, a point can belong to multiple clusters. This belonging (also known as membership) is a percentage (e.g., a point $p$ membership maybe be $30 \%$ to cluster A, $60 \%$ to cluster $\mathrm{B}$, and $10 \%$ to cluster $\mathrm{C}$ ).

The belonging of each data point (to multiple clusters) is represented as a vector of values between 0 and 1 , inclusively. This vector is often referred to as the membership vector. The values in each membership vector should sum to 1 . The values in the membership vectors reflect how much it belongs to the corresponding cluster; the closer the value to 1 , the stronger the belonging is to that cluster.

In Table 2.2, we show the notation and variable names, which are the same as the ones presented by Bezdek et al. [10].

\begin{tabular}{cl}
\hline Notation & Description \\
\hline$m$ & weighting exponent to control fuzziness \\
$y_{k}$ & the data point value at index $k$ \\
$v_{i}$ & cluster at index $i$ \\
$d_{i k}$ & the distance between a data point $y_{k}$ and a cluster center $v_{i}$ \\
$N$ & the number of data points \\
$c$ & number of clusters \\
$u_{i k}$ & the membership value of a data point at index $k$ to cluster $i$ \\
\hline
\end{tabular}

Table 2.2: FCM notation and variable names

The workflow of the FCM algorithm is as follows:

1. Fix $c, m$, and $U$ (initialization).

2. Compute centers for each cluster.

3. Update membership matrix $U$. 
4. If the change in $U$ is significant, go to step 2 .

\subsubsection{Initialization}

In this step, we fix the number of clusters $c$ and the weighting exponent of the fuzziness $m$. There is no theoretical or experimental evidence on the optimal value of $m$, but Bezdek et al. [10] reported good results most of the time for $m$ between 1.5 and 3 . The values of the membership matrix $U$ are set randomly (ensuring that the membership vector of a given point sums to 1).

\subsubsection{Cluster Center Computation}

The center for each cluster is computed using Eq. 2.1. The cluster centers are not fixed and may change depending on the membership matrix, which may change as well (reducing the total squared error).

$$
v_{i}=\sum_{k=1}^{N}\left(u_{i k}\right)^{m} y_{k} / \sum_{k=1}^{N}\left(u_{i k}\right)^{m} \quad ; 1 \leq i \leq c
$$

\subsubsection{Membership Assignment}

The membership is (re-)computed every time that the cluster centers are changed. The membership matrix is computed using Eq. 2.2. The new membership matrix is compared with the previous membership matrix. If the difference is significant (more than a given threshold), the cluster centers are re-computed again (the algorithm continues to loop). Otherwise, the algorithm terminates, and the membership matrix is returned.

$$
u_{i k}=\left(\sum_{j=1}^{c}\left(\frac{d_{i k}}{d_{j k}}\right)^{2 /(m-1)}\right)^{-1} ; 1 \leq k \leq N \quad ; 1 \leq i \leq c
$$




\subsection{Summary}

In this chapter, we have introduced what we consider background knowledge that can help in understanding better the state of the art analysis that we included in Chapter 3, the design of our research as presented in Chapter 4, and the main contributions of our work as presented in Chapters 5 and 6 .

We have discussed the term knowledge graph since it will be used by our approach as a source of knowledge to derive annotations, as well as about the different forms in which tabular data may be made available and the main types of columns that we can find. Finally, we have described briefly what we understand by clustering and have provided some details of the clustering techniques that we will use in one of our contributions. 


\section{Chapter 3}

\section{State of the Art}

The area of semantic labeling has evolved from different areas and problems, such as entity linking/disambiguation, search engines, and tabular data linking and annotation. Different approaches have been proposed so far to understand tabular data; some annotate them with semantic types from ontologies, others link similar data without labeling them with semantic types. Here we describe the most relevant approaches reported in the literature.

\subsection{Techniques}

In this section, we describe the different techniques we encountered in the literature. Some of these techniques use machine learning, and others do not. For the machine learning techniques, we group them, following Flack [30], into three groups: geometric, probabilistic, and logical. We add another group for non-machine-learning techniques. In case multiple machine-learning techniques are used, the approach is classified based on the best performing technique in the published work. We order the approaches in the same category based on the time on the publication year. 


\subsubsection{Geometric Techniques}

Cafarella et al. [14] proposed an approach that uses linear regression. It starts by crawling the Web for tables. It distinguishes HTML tables used for positioning and HTML tables that contain tabular data using [15]. From the tabular data ${ }^{1}$, it constructs the attribute correlation statistics database (ACSDb). This database includes the frequency for each schema in the tables. It permits the computation of probability of an attribute given another attribute. The aim is to search for relevant tables given some terms. To return the most relevant tables, they proposed different ranking methods. The first two rely on the Google search engine. The third one uses several features, such as the number of hits on the header, the number of hits on the leftmost column, and the number of hits on the table body. The fourth rank method uses these features and additionally uses Point-wise Mutual Information (PMI). It measures the relationship between two items using the ACSDb (e.g., how strongly a and $b$ are related). Finally, they use Linear Regression over those features. The training is done using more than a thousand pairs of (query, table), which was evaluated by two human judges. Their tests revealed that using PMI and the other features outperforms the search engine performance on tabular data.

Quercini et al. [61] experiment with Google Fusion Tables (GFT). Their approach focuses on columns that contain entities. To detect such columns, it ignores cells that most probably do not represent entity labels (e.g., restaurant names). It uses regular expressions to detect cells with phone numbers, URLs, email addresses, numeric values, and geographic coordinates. Such cells are ignored. It also eliminates cells that contain long text (e.g., description). It utilizes the GFT typing feature to exclude cells with GFT types such as Location, Date, or Number. For all the cells that are not excluded, the Bing search engine is used. Microsoft Bing returns a list snippets for each cell; the top-k results are considered. Each snippet is converted to lower-case, tokenized, and stemmed. The relative frequency of each token in each snippet is computed. The number of snippets that are classified as a description of an entity of a given type $t$ is computed. This is performed for all the types - which are 12 manually

\footnotetext{
${ }^{1}$ Cafarella et al. [14] refer to tables as relations in their paper
} 
picked classes from DBpedia - and the most common type is selected. If that selected type covers less than half of the selected snippets, that cell will not be annotated. Otherwise, that cell is assigned to the commonly predicted type. The approach also uses spatial information in the tables using the Google Geocoding API service. The authors compared SVM with Naive Bayes, and they obtained better results using SVM. The results show that the proposed approach is promising to perform semantic labeling on tabular data.

Pham et al. [56] proposed approach aims to perform semantic labeling. It uses the similarity of attribute names and the Mann-Whitney test for the histogram of the values. For textual values, it uses Jaccard similarity and TF-IDF cosine similarity. For numeric values, it uses a modified Jaccard similarity (based on the range of values) and Kolmogorov-Smirnov test. Given a set of labeled data and unlabeled data, the aim is to use the labeled data to learn a model and then use it to annotate the unlabeled data. They experimented with a collection of datasets in the following domains: city, weather, museum, and soccer. They used the T2D gold standard in their other experiment. They reported the performance of their approach using the mean reciprocal rank (MRR). They compared their approach using two techniques: Logistic Regression and Random Forests. The experiments show that the Logistic Regression technique outperformed the Random Forests one. The results show that

this approach is promising to perform semantic labeling on using a learned model to assign labels to a new dataset.

\subsubsection{Probabilistic Techniques}

Limaye et al. [45] proposed an approach to perform semantic labeling. Their approach uses graphical models with several potential functions. It uses TF-IDF cosine similarity to match the cell value to the most probable entity. It also uses the same similarity function to match a given column header with the most similar type. Another potential is the IDF measure; it divides the number of entities by the number of entities of a given type. It uses the distance between the entity and its type, formalized as the reciprocal of the square root of the distance (between the entity and 
its type). For the relation between two columns, it examines the two types of the two columns in the catalog. It also computes the fraction of entities in the catalog that have the relation between the two types (of the two columns). The last potential checks whether there is a relation between two given entities. Each one of these potential functions is weighted. These weights are computed using SVM, with tables crawled from Wikipedia. For the annotation task, they experimented with crawled tables from Wikipedia (the same tables used to compute the weights) and the Web with YAGO as the catalog. The results show that the proposed approach is promising to perform semantic labeling. They also presented a search application with multiple cases: without annotations, with type annotation, and with type and relation annotations. They used DBpedia for the evaluation of the performance of the search. The experiments show that using the type and relation annotations outperforms the other two cases.

Venetis et al. [80] aim to add annotations to columns in tabular data and relations between columns. In their approach, a single column can have multiple annotations. First, they construct the isA database. This database consists of (instance, class) pairs. It is built using simple linguistic patterns applied to textual data (e.g., "...cities such as Madrid"). Then, the probability of different labels given the values of a given column is used (maximum likelihood). This is done using the is $A$ database. For extracting the relation between columns, they used an external system called TextRunner. For the experiments, the $i s A$ database is build applying linguistic patterns over 100 million English documents. The experiment found around 60,000 classes with at least 10 instances. For the tables to be annotated, a corpus of 168 of highquality tables is picked from a Web crawl. They compared their approach with the majority approach (picking labels that cover more than half the cells for a given column). Then, they experimented with a hybrid approach of these two. The experiment showed that the hybrid approach outperforms both of the other approaches.

Goel et al. [31] proposed approach is based on graphical models. They use several feature functions and weight each function using the gradient. Cell values are tokenized at white spaces. Each token is further divided (if possible) such that each part 
is fully alpha (e.g., cloudy), numeric (e.g., 32), or a symbol (e.g., \%). The features are different for each type. The features for the alpha type are the length of the token, the first letter, whether the token is capitalized, whether all the letters are capitalized, and then the token value itself. For the numeric type, the functions are the length of the number, the number of digits before the decimal points, the number of digits after the decimal point, whether the number is negative, the starting digit. For the symbol, only the symbol value itself is used as the feature. Each cell value is associated with features. For example, if we have a cell value "cloudy", the following features are linked to it: starts_with_alpha_c, alpha_length_6, alpha_id_cloudy. The feature function takes the value with its equivalent label (e.g., cloud coverage) as the parameters. Also, the order of fields is taken into account with their equivalent labels. The parameters to that potential function are the two values with their equivalent labels. Taking the order of values into account improves the accuracy as it helps in the disambiguation of similar fields. Finally, the inference is performed using the belief propagation algorithm, and the prediction is performed using the Viterbi algorithm. The experimented with data crawled from the web. They divided their data into training and testing sets. They compared their approach with a regular expression based approach and showed that the performance of their approach is better than the regular- expression-based approach.

Zhang et al. [84] proposed an approach to match numeric values, entities, and columns in tabular data to improve search quality. The approach focuses on timevarying numeric values (e.g., the population of Madrid in 2019). The approach annotates each numeric column with a year, a scale (e.g., million), and a unit (e.g., Euro). The input tables are converted into entity-attribute binary tables (EAB). A table with $n$ columns is divided into n-1 EAB tables such that each of them consists of the subject column and an attribute column. The system includes a set of handcrafted conversion rules to convert values from one unit or scale to another (e.g., Euro to US Dollar). A table is annotated with some labels (e.g., USD, billion) and a year (e.g., 2019) using the header of the table and the context (e.g., caption, surrounding text). The set of possible labels are taken from the conversion rules. The approach 
only considers the year range from 1900 to 2050. The SU matching potential function is about the matching between two EAB tables. If two tables share similar entities and the values almost match (the difference is less than a threshold) directly or after applying conversion rules, then they would match. There is also the SU and year propagation functions. If two EAB tables are matched, the year, the SU labels, and related conversion rules (if applicable) are propagated from one table to the other. The authors experimented with a subset of web tables extracted from a snapshot of Microsoft Bing. They picked three domains: Company, Country, and City. Their aim is to improve search results. They compared their approach using the probabilistic approach against baselines using only the SU labels and the years. Their experiments show that their probabilistic approach outperforms the baselines significantly.

Mulwad et al. [50] aim to semantically annotate cells and columns of tabular data found on the Web. The approach starts by detecting types, such as SSN, zip codes, phone numbers, and addresses. It generates an initial list of candidate entities using Wikitology and ranks them based on the work of Dredze et al. [23]. Potential classes of a column are the union of the classes if its cells. To detect the relation between two columns, the relation between their entities are collected using DBpedia and Yago. The union of these relations between the entities of the two columns is gathered as potential relations between the two columns. Cells that do not represent entity mentions are ignored. For the inference, the approach uses an inference algorithm based on the message passing technique. The approach uses two functions. The first one computes the most potential entities using the potential classes for the column header. In case one of the suggested entities does not agree with the potential classes, the function will ask for an alternative entity. The second function is used to discover relations between columns. The relation between the two columns is detected using the relation between the entities of the two columns. The majority vote is picked. They experimented with tables crawled from the Web, and it shows that their message-passing approach outperforms the approach of Limaye et al. [45].

Ermilov et al. [26] presented TAIPAN to tackle two problems: subject column identification and property mapping. For the subject column detection, the approach 
uses support and connectivity. They define support of a column as the ratio between the number of disambiguated cells and the total number of cells in that column. The disambiguation is performed using AGDISTIS [79] with DBpedia as the reference knowledge graph. The connectivity is to measure the relation of a column with the other columns. This is done using the relation between the disambiguated entities of a column and the other cells of the other columns. For the property mapping, each table is split into $n-1$ tables, where $n$ is the number of columns. The subject column is matched to each of the other columns to form n-1 tables. Each table would be composed of the subject column and a column such that the relation between the subject column and the other column corresponds to a (property) relation in the knowledge graph. Then, a probabilistic model is employed using the data within the table and the relations in the knowledge graph. The experiment is performed on a selected subset of the T2D dataset (Web Data Commons) and DBD (DBpedia Table Dataset). For subject column identification, more features (besides the support and connectivity) were considered, such as the fraction of unique cells, the fraction of numeric cells, and column index from the left. The authors experimented with several machine learning techniques, such as SVM, KNeighbors, and Decision Tree. SVM performed best on the T2D dataset while the KNeighbors technique performed best on the DBD dataset. The Decision Tree achieved better than KNeighbors on the T2D and better than SVM on DBD. For the property mappings, TAIPAN is compared against T2K and achieved a higher F1 score on both, T2D and DBD datasets. The experiments show that using the support and connectivity features improves the performance of subject column detection and that the TAIPAN approach outperforms T2K (on the used dataset).

\subsubsection{Logical Techniques}

Syed et al. [75] presented Wikitology: a knowledge graph constructed from Wikipedia. It integrates knowledge graphs, such as DBpedia, YAGO, and Freebase. The presented approach uses Wikitology to perform entity linking (and semantic labeling). It takes an entity name and queries Wikitology to match it with a Wikipedia article. 
It also uses the table header and tries to match it with classes from DBpedia, YAGO, WordNet, and Freebase. It also uses the first sentence in Wikipedia as well. Potential entities and their classes are gathered for each cell. The approach assigns a score of each potential entity as a weighted sum of the reciprocal of the entity rank and an approximated page rank. The most occurring class is used to be the class of the whole column. The predicted class is then used to improve the entity linking. The candidate relations between two columns are gathered using the relation between the entities in the two columns. The majority relation is then selected. The page rank is approximated from the Google search engine. The authors selected a number of random articles from Wikipedia and used Google to get their page rank. The used in- and out-links (to and from the Wikipedia article), and the article length as the features. 10 fold cross-validation is used to compare the page rank performance with the following techniques: SVM, Decision Trees, and Naive Bayes. Decision Trees resulted in the highest accuracy for the page rank. The experiments showed that their approach is promising in performing semantic entity linking and semantic labeling.

Tonon et al. [77] aim to predict the most relevant type (class) given an entity in a document. The approach uses a conditional-random-fields technique [29] for entity identification. There are different kinds of context used, such as the sentence containing the entity mention and the paragraph containing the entity mention. The system relies on a large type hierarchy based on previous ontology alignment, combining type hierarchies from different ontologies: DBpedia, YAGO, and schema. The resulting type hierarchy was updated manually and reviewed by domain experts. The authors presented several techniques to predict the most relevant classes to entities. Some of these techniques do not take into account the context and rely on features such as the frequency of the occurrence of the class (e.g., person is more frequent than blogger) in the knowledge graph, the relation between entities using wikilinks and sameAs properties in the knowledge graph (neighboring entities share the same type) and TF-IDF similarity. The second type takes the context into account; such techniques are called context-aware. Such techniques takes into account entities appearing in the same context and sharing the same path (in the type hierarchy) to be of the same class. The 
last kind is referred to as hierarchy-based. One technique uses the type hierarchy and prefers the deepest class in the type hierarchy. The other technique checks for types with similar ancestors. For the experiments, the authors selected news articles from the New York Times. The results show that hierarchy-based techniques outperform the rest. The authors also experimented with a combination of those techniques using Decision Trees and Linear Regression. The results showed that using the Decision Trees outperformed the Linear Regression technique and the other techniques. This study shows the importance of type hierarchy for assigning the most relevant classes for entities.

Neumaier et al. [52] propose an approach to label numeric values using a given knowledge graph. The approach utilizes the context to assign the semantic label to a given bag of numbers. The context is a set of attributes and values (e.g., basketball players). The approach queries the knowledge graph for numeric properties by checking the properties with numeric values. A tree is constructed for each of those numeric properties with the property as the root node (referred to as background knowledge graph). Subjects that share the same numeric property are grouped (to relate the numeric property node). Subject that belongs to a node can be split further, such that each of these sub-groups shares a more specific context (e.g., the context of basketball players is more specific than the context of the class person). Each of these contexts would be a node that is a child of the root node and can be split further to more specific context nodes. For the prediction, different distance measures are used. For a given a bag of numbers, the approach compares the distance between the given bag of numbers and the closet context node. For the experiment, the 50 most used numerical properties in DBpedia are selected and are used to build the background knowledge graph. The subjects from DBpedia are divided into a training set of $80 \%$ of the data and $20 \%$ for the testing set. The experiments show that the KS test outperforms the other distance measures. The authors also experimented with tabular data from two open data portals: the Austrian Open Government Data portal and the European Open Data portal. To get more from the headers, they are pre-processed and mapped using BabelNet APIs. The results show that it is difficult 
to match such tables to DBpedia. Possible reasons are time-varying properties (data which change over time, such as the population) and the limited coverage of DBpedia.

\subsubsection{Non-Machine-Learning Techniques}

Deng et al. [21] presented an approach that assigns a type to a given column using the knowledge graph. It utilizes the similarity between the given entity column and the entities of the equivalent type in the knowledge graph. They presented the following similarity functions to compare the similarity of two sets: overlap similarity, Jaccard similarity, Cosine similarity, Dice similarity, and weighted Jaccard similarity. These set similarity functions use exact-match when matching two elements from the two sets. The authors extended these similarity functions using fuzzy-match instead of exact-match in these similarity functions. Fuzzy match is to match two elements that might not be exactly the same. Map-Reduce framework is used to improve the scalability. To further improve the performance, types are aggregated into groups. These groups are used to reduce the number of key-value pairs (which is the way Map-Reduce based applications pass data). Another optimization is the use of a partition framework. All values in the different columns are gathered into one vector and then split into $p$ disjoint partitions. The partitions are mapped to the columns containing the cells within. These partitions are then typed, and then fuzzy similarity functions are applied to type the related columns. The authors experimented with tables extracted from Wikipedia. Freebase and Yago are used as knowledge sources. The authors compared the results of the different similarity functions of their approach with the approach of Limaye et al. [45]. The proposed approach outperformed the approach of Limaye et al. [45] in recall, performed worse in precision, and achieved a similar F1 score. So, the proposed approach is scalable while achieving similar performance to the approach by Limaye et al. [45].

Zhang [85] proposed an approach to add semantic labels to entity columns using a given knowledge base and exploiting context, such as RDFa. First, the approach utilizes a predefined set of regular expressions to detect entity columns, such as the capitalization, numbers, and the number of tokens in cells. Context used includes 
captions of the tables, titles of the publishing Web page, surrounding text, and semantic markups (RDFa). The potential entities for each cell are disambiguated using the context of the table. Then, potential classes are gathered from the classes of the potential entities of the column. For each candidate entity of a cell, patterns of the candidate entity are queried from the knowledge graph. These patterns are then tokenized, lemmatized, and cleaned from the stop words. The resulting bag of words is concatenated and compared with the context of the table. Weighted dice measure is used to compare the similarity. The second measure computes the overlap between the bag of words of the cell content and the entity. The third measure uses a weighted dice function between the bag of words of the header text of the column and the bag of words of the potential classes. The iteration continues until convergence when the change in the candidate classes is less than a threshold. The same process is repeated while restricting the disambiguation of entities to the top candidate classes of the column. If the top candidate classes differ from the previously selected ones, the process is repeated until there are no changes. The author experimented with two datasets: one is composed of tables from Wikipedia, and the other contains tables extracted from IMDB (Web pages of movies). Freebase was used as the knowledge source. The results show that the proposed approach has the potential to perform semantic labeling of entity columns. The results also show that the use of context (e.g., table caption, text surrounding the table, $\mathrm{RDFa}$ ) is very valuable in the semantic labeling of tabular data.

Ritze et al. [64] present an approach to perform semantic labeling. They also present the T2D gold standard, which contains manually annotated tables crawled from the Web. As the data are crawled from HTML pages, the HTML tags and special characters are removed, and the values are lower-cased and normalized (e.g., resolving abbreviations and units of measurements). Data types (e.g., numbers, coordinates) are identified using manually defined regular expressions. The data type of a column is the majority predicted data type of its cells. A subset of DBpedia is used as the external source of knowledge to be used for entity linking and semantic labeling of the tables. Candidate entities for each cell are selected by querying DBpedia for 
entities with labels that match the cell value (using Jaccard similarity). The most frequent class of the suggested entities in the cells of the entity column is picked to be the class of the column. Then, candidate entities for the cells are disambiguated, such that entities that do not share the same class as the selected class (of the column) are eliminated. Values in other columns are matched to property values from DBpedia. The Levenshtein distance is used to match string values and deviation similarity for numeric values. The candidate with the highest score is selected with its equivalent property from DBpedia. The property with the highest score is selected and aggregated per class to refine class ranking. The algorithm iterates as candidate classes and properties influence each other. The loop terminates when the similarity scores are no longer changing. Their gold standard - which are the tables that were manually annotated - were used in the experiment. The performance of semantic labeling and entity linking were measured using precision, recall, and F1 score. The results show the potential of the proposed approach to perform semantic labeling and entity linking.

Ramnandan et al. [62] proposed an approach to perform semantic labeling on textual and numeric data. For textual data, TF-IDF cosine similarity is used to measure the similarity. For numeric data, the distribution is compared using the Kolmogorov-Smirnov (KS) test. For cases where the data contain a mix of both, both techniques are used. The experiments were performed with several datasets. For the textual data, datasets from art museums in the U.S were used. For numeric data, numeric properties were extracted from DBpedia from the class city. For the mixed case (textual and numeric data), properties from the city class were extracted from DBpedia. For each of the cases (textual, numeric, or mix), learning was performed on a different number of datasets, and the testing was performed on one that was not included in the training. For each dataset, the experiment was repeated n-1 times (where $\mathrm{n}$ is the number of datasets). Each of these runs was performed with a different number of training sources, enumerating from 1 to $n-1$. The scores were averaged, aggregated by the number of datasets used. The score measure used was the mean reciprocal rank with $\mathrm{k}=4$ (the top 4 candidates). For the textual data, the TF-IDF 
cosine similarity was compared against the Jaccard similarity and CRF (by Goel et al. [31]). TF-IDF outperformed the other two. For the numeric data, the properties extracted from DBpedia (of the class city) was divided into 10 datasets. The KStest was compared with Mann-Whitney U-test, TF-IDF cosine similarity, Welch's t-test, and CRF [31]. The KS-test outperformed all the other approaches. For the mixed data case (numeric and textual), the approach was compared with CRF [31] and TF-IDF cosine similarity. The proposed approach (combining TF-IDF and KStest) outperformed the others. The experiments showed that TF-IDF similarity and KS-test are powerful to perform semantic labeling in comparison with the others.

Taheriyan et al. [76] aim to create a semantic model of a given dataset using manually crafted semantic models created by experts. Such semantic models include instances, classes, and properties. The idea is to exploit the overlapping of data from the known semantic models and the target dataset to infer classes, properties, and relations. The first step is to perform semantic labeling of the new dataset using semantic models. For textual data, TF-IDF cosine similarity is used, and for numeric data, the distributions are compared. The second step is to build a graph from the known semantic models, semantic types, and domain ontology. It creates an empty graph and adds nodes and links from the semantic models if they do not exist. Then, it adds semantic types to the instances in the new dataset and missing classes and properties from the domain ontology (e.g., properties inferred from the sub-class hierarchy). The approach adds weights to links, preferring (assigning less weight to) popularity and links from known semantic models to links from the domain ontology. In the third step, it infers mappings using heuristics to map semantic types to nodes. The output is a set of candidate mappings between attributes from the source (learning set) to the graph. The fourth step is generating and ranking semantic models. The semantic model of a mapping is the tree with the least cost (weight) that connects the nodes in the mapping. As this task is costly, an approximation is used instead. The authors experimented with several datasets from different art museums of the United States. Two known models to represent museum data are used: the Europeana Data Model and the Conceptual Reference Model. These two 
use several domain ontologies. The semantic labeling results are reported using the mean reciprocal rank MRR. Semantic models are reported using precision, recall, and F1 score, comparing them to manually created semantic models. The results show that the proposed approach is promising to perform semantic labeling and semantic modeling using previously constructed semantic models.

Zhang [86] extends his previous approach [85] to improve accuracy. Two kinds of contexts are considered: in-table context and out-table context. The former includes column header, row content, and column content. The latter consists of the Web page title, table caption, surrounding paragraphs, and semantic markups (RDFa). The approach detects subject columns using regular expressions and utilizes other features, such as the fraction of empty cells, the fraction of unique cells, the distance to the first entity column, the context match, and the web search score. The context score is the weighted frequency of the header's words in the context. The web search score uses the Microsoft Bing search engine to count the frequency of the cell content in the title and the snippet of the top web pages resulted from the query - which is a concatenation of the values of the cells in a row. This process is repeated for multiple rows. These different features are then normalized and are used to identify the subject column. The same features are used to perform semantic labeling of entity columns with extra features, such as the similarity between the entity name and the cell content, and the context overlap with patterns from the knowledge base. Also, the relations between the subject column and other columns are detected using the relations between the underlying cells and the knowledge base. The author experimented with datasets from Limaye [45] (the author rebuilt this dataset as it is not published), IMDB (Web pages of movies), and MusicBrainz (Web pages about music). Freebase is used as the knowledge base for annotating the tables of those datasets. The performance of the subject column detecting is evaluated using precision. For semantic labeling (and entity disambiguation), the performance is measured using the F1 score. The results show that the proposed approach outperforms the state-of-the-art approaches included in the experiment. The results also show that the use of context is valuable for entity disambiguation and semantic labeling. 
Ritze et al. [63] propose an approach to address three tasks: entity linking, property matching, and class matching. To perform entity linking the subject column (which contains the entities) need to be identified. A heuristic relying on the number of unique values in a column is used. Several features are used, such as Levenshtein distance, deviation similarity (for numeric values), set-based comparison with synonyms and hypernyms using WordNet, and cosine similarity of the abstracts of the potential entities. For property matching, the following features are used: Levenshtein distance (to compare header of a column to the property in DBpedia), comparison with synonyms and hypernyms using WordNet, and a dictionary created from connecting tables from Commons Web Tables Corpus to DBpedia, and the similarity of entity-property pairs. The last task is class matching; it assigns a class from DBpedia to the subject column of the table. Several features are used, such as page title and URL of the page containing the table, the specificity of the classes (in the class hierarchy), and the count number of the classes by the candidate entities. The authors experimented with version 2 of the T2D gold standard, which contains manually annotated tables crawled from the Web. To measure the performance of the approach, the precision, recall, and F1 score are measured for the different combinations of the matchers (features) for the different tasks (entity matching, property matching, class

matching). The best combination of matchers for the property matching task is the Dictionary matcher with the Duplicate attribute matcher. For the entity matching and class matching, combining all the matchers produces the best performance. The results show that combining the different features reported in the literature improves the overall semantic labeling scores. The authors also warn from the use of some of them (e.g., WordNet for property matching) might introduce noise, which may reduce the overall performance.

\subsection{Data for Evaluation}

This section discusses about the data that have been used for training and/or testing some of the aforementioned approaches. In Table 3.2, we list the data that are 
Table 3.1: Summary of the different approaches with their techniques

\begin{tabular}{|c|c|c|c|}
\hline Approach & Technique & Learning Sources & $\begin{array}{l}\text { Column } \\
\text { Types }\end{array}$ \\
\hline Cafarella et al. [14] & linear regression & Crawled Web Tables & all \\
\hline Limaye et al. [45] & graphical models & $\begin{array}{l}\text { Wikipedia, DBpedia, } \\
\text { YAGO }\end{array}$ & entity \\
\hline Syed et al. [75] & decision tree & Wikitology & entity \\
\hline Venetis et al. [80] & maximum likelihood & Web pages & entity \\
\hline Goel et al. [31] & graphical models & Web pages & all \\
\hline Zhang et al. [84] & graphical models & Web pages & $\begin{array}{l}\text { entity }+ \text { nu- } \\
\text { meric }\end{array}$ \\
\hline Tonon et al. [77] & decision tree & $\begin{array}{l}\text { YAGO }+ \text { DBpedia } \\
+\quad \text { Sindice-2011 }+ \\
\text { schema.org }\end{array}$ & entity \\
\hline Quercini et al. [61] & SVM & DBpedia & entity \\
\hline Deng et al. [21] & non-ML & Freebase, YAGO & entity \\
\hline Mulwad et al. [50] & graphical models & $\begin{array}{l}\text { DBpedia, } \\
\text { Wikitology }\end{array}$ & entity \\
\hline Zhang [85] & non-ML & Freebase & entity \\
\hline Ritze et al. [64] & non-ML & DBpedia & all \\
\hline Ramnandan et al. [62] & non-ML & $\begin{array}{l}\text { DBpedia }+ \text { Web pages } \\
+ \text { museum dataset }\end{array}$ & all \\
\hline Pham et al. [56] & logistic regression & $\begin{array}{l}\text { DBpedia }+ \text { Web pages } \\
+ \text { museum dataset }+ \\
\text { T2D }\end{array}$ & all \\
\hline Neumaier et al. [52] & hierarchical clustering & DBpedia & numeric \\
\hline Taheriyan et al. [76] & non-ML & $\begin{array}{l}\text { museum dataset }+ \\
\mathrm{EDM}+\mathrm{CIDOC}\end{array}$ & all \\
\hline Ermilov et al. [26] & probabilistic model & DBpedia & $\begin{array}{l}\text { textual } \\
\text { numeric }\end{array}$ \\
\hline Zhang [86] & non-ML & Freebase & all \\
\hline Ritze et al. [63] & non-ML & DBpedia & $\begin{array}{l}\text { entity } \\
\text { string } \\
\text { numeric } \\
\text { date }\end{array}$ \\
\hline
\end{tabular}


linked/annotated by the different approaches. We mention whether they are published (by searching for references in the articles), accessible (by checking whether we can get to the data), and easy to use (whether the data are described, and the format is easy to work with like CSV). We can see that the only annotated data that are published, accessible, and described so they are easy to use are T2D (version 1 and version 2) by Ritze et al. We will be using the newer version of T2D (T2Dv2), as it contains more data. Therefore, this will be one of the datasets that we will make use of in our work, together with new one that will be presented in Section 4.3.

\subsection{Limitations}

From our analysis of the related approaches to the domain of semantic labeling, we summarize our observations as follows:

- The absence of semantics: some approaches connect tabular data together. They link data that represent the same entity or concept without identifying the actual meaning of each $[14,15,84]$. In other words, these approaches link similar data rather than annotating them with semantic types. Such approaches do not ensure the interoperability of the different data that they connect. In our approach, we will annotate datasets with semantic types from a given knowledge graph, what may make it much easier to exploit and integrate with other datasets (having the advantage of data interoperability) [64, 52].

- The reliance on search engines: some approaches use Web search engines beside knowledge graphs to disambiguate entity linking, such as Zhang [86] and Quercini et al. [61], which makes them dependent on and bound to the search engine used. Our approach will not rely on search engines as an additional source of information besides a given knowledge graph.

- The need for profiling beforehand: some approaches require building a model before being able to annotate input data $[52,75]$. Sometimes they need to build an external index, such as Tonon et al. [77], where an inverted index is built 
Table 3.2: Data that are linked or annotated for the different approaches

\begin{tabular}{llccc}
\hline Approach & Data (to be annotated) & Published & Accessible & Ease of use \\
\hline Cafarella et al. [14] & Google Crawl & No & No & $?$ \\
Limaye et al. [45] & Their Web Crawl & No & No & $?$ \\
Syed et al. [75] & Tables from Google Square & No & No & $?$ \\
Venetis et al. [80] & Their Web Crawl & No & No & $?$ \\
Goel et al. [31] & Their Web Crawl & No & No & $?$ \\
Zhang et al. [84] & Bing Crawl & No & No & $?$ \\
Tonon et al. [77] & Their Web Crawl & Yes & No ${ }^{\dagger}$ & $?$ \\
Quercini et al. [61] & Extract from Google Fu- & No & No & $?$ \\
& sion Table & & & \\
Deng et al. [21] & WWT + WEX & Yes & No ${ }^{\dagger}$ & $?$ \\
Mulwad et al. [50] & Web Crawl & No & No & $?$ \\
Zhang [85] & Tables from Wikipedia and & No & No & $?$ \\
& IMDB & & & \\
Ritze et al. [64] & Web Data Commons & Yes & Yes & Yes \\
& (T2D) $*$ & & & \\
Ramnandan et al. [62] & Hand picked tables & Yes & Yes & No \\
Pham et al. [56] & Hand picked tables + T2D & Yes & Yes & No \\
& $*$ & & & \\
Neumaier et al. [52] & Extract from DBpedia & Yes & No ${ }^{\dagger}$ & $?$ \\
Taheriyan et al. [76] & US Art Museums & Yes & Yes & No \\
Ermilov et al. [26] & DBD + T2DT \\
Zhang [86] & Tables from Wikipedia + & Yes & Yes & No \\
Ritze et al. [63] & IMDB + MusicBrainz & Yes ${ }^{\ddagger}$ & $?$ \\
\hline
\end{tabular}

${ }^{*}$ These are gold standards published by Ritze et al. [64, 63].

$\S \mathrm{T} 2 \mathrm{DT}$ is a subset taken by Ermilov et al. from the Data Web Commons published by Ritze et al.

We are not referring to the T2D dataset here (which is already described). We are referring to the other datasets that are made available in GitHub as pointed out in the paper.

" Google Square and Google Fusion Tables were public, but they are no longer accessible during the time of writing.

$\dagger$ Invalid URLs.

$\ddagger$ Although the data are not published in the paper, the author actually mentioned in a footnote of the paper that he can provide the data if asked by an email because the data are large to host.

? Unknown 
over the whole DBpedia to increase performance. This is usually expensive in terms of storage and time. Our approach creates the model once the input file is provided, being so small that it only contains semantic types related to the provided file.

- The dependence on fixed data sources: some approaches are bound to a specific data source. For instance, the work by Syed et al. [75] relies on Wikipedia and Wikitology (even though this index may be rebuilt again with other similar sources, it is a complex process). Tonon et al. [77] rely on PARIS [74] for the alignment of the type hierarchy. In our case, we can use any existing knowledge graph, as long as it is made available in RDF and accessible via SPARQL, since our approach does not perform any profiling for the knowledge graph. Some approaches share the same advantages as ours, using knowledge graphs as learning sources, such as YAGO in [45] and DBpedia in [52, 64], which makes them flexible and applicable to a broader range of training sets (data to learn from).

- The need for manual intervention: some approaches require manual intervention (human in the loop) as part of their workflow. Despite the fact that these approaches may be automatic or semi-automatic ${ }^{2}$, some of them actually require manual actions (e.g., provide predefined conversion rules [64, 84], manually tweak the type hierarchy [77], ontology alignment [62], a blacklist of properties [52] to improve the accuracy, and abbreviations resolution [64, 84]). Our approach is completely automatic and does not contain such manual crafting, which makes it more easily adaptable to different datasets and knowledge graphs.

In summary, we will address several relevant limitations in the state of the art of semantic labeling of tabular data as follows. First, emphasize on the interoperability of annotations using semantics (ontologies adopted in knowledge graphs). Second, en-

\footnotetext{
${ }^{2}$ We are not referring here to the gold standards that are built manually for evaluation purposes or the semantic models that are constructed by domain experts
} 
sure the independence of other external sources of information (e.g., search engines). Third, circumvent profiling of knowledge graphs beforehand (e.g., build inverted index). Last, avoid manual tasks in the workflow (e.g., tweak the class hierarchy). 


\section{Chapter 4}

\section{Research Design}

In this chapter, we present our research questions and hypothesis, such that, their answers contribute to the evaluation of our research hypothesis. We designed our research to answer these questions quantitatively, using widely used metrics like precision, recall and F1 score. We also present the assumptions we considered in this work and follow that by the known limitations of our approach.

\subsection{Research Questions and Hypothesis}

From the analysis of the literature on semantic labeling that we presented in Chapter 3, we have observed several downsides, such as the lack of semantics (due to the use of unlabeled data), the reliance on search engines, the boundness to specific data sources, the need for profiling data sources before performing semantic labeling, and the need for manual tasks such manual adjustment of the type hierarchy. As a result of such analysis of limitations, we propose a way to overcome them.

\section{Research Questions}

Q.1 Is there a way to match automatically the majority of subject columns of tabular datasets with the corresponding classes in knowledge graphs, without considering the context in which the data appear, and without using other external sources of information and knowledge graph profiling beforehand? 
For example, let us suppose that we have a table with the subject column containing the following values: "Marie Curie", "Charles Darwin", and "Charles Lyell". Given a knowledge graph (e.g., DBpedia), can we conclude that this column is represented by the class dbo:Scientist ${ }^{1}$ ? In this research question, we try to address the semantic labeling problem of subject columns - columns that contain the labels of the main entities. The key is to perform semantic labeling without the limitations we observed in the literature. Some approaches rely on context such as the URL where the dataset is made available, the caption of the table, or the text surrounding the table. Another limitation is the reliance on external sources of information such as linguistic resource and search engines. The third limitation is the need to profile the knowledge graph (perform some computations) before the semantic labeling be applied. The last limitation is the need for manual tasks (e.g., the need to adjust the type hierarchy).

Q.2 Is there a way to automatically match most of the numeric columns in tabular data to their corresponding properties in knowledge graphs, without considering the context in which the data appear, without using other external sources of information and without performing entity linking given the type of the subject?

For example, the knowledge graph may contain the heights of Italian football players and the input tabular data may include some numeric values of the Spanish football players. Is there a way to identify the numeric values associated with the Spanish football players as their heights, given that we know they are football players? This research question addresses the problem of semantically labeling numeric columns in tabular data, to match them with properties from a given knowledge graph. The aim is to perform semantic labeling while overcoming the limitations we observed in the literature (Section 3.3). This is without performing entity linking given the semantic type of the subject.

Q.3 Does taking the numeric typology into account improve the F1 score of semantic labeling to assign semantic labels from a given knowledge graph to numeric

\footnotetext{
${ }^{1} d b o$ is a prefix that is short for http://dbpedia.org/ontology.
} 


\section{columns in tabular data?}

For example, let us suppose that we have two columns. The first column contains heights of football players, and the second one contains the number of goals they scored in a season. Does taking into account that these two numeric columns are of different numeric types ${ }^{2}$ and treating them differently, yield better semantic labeling results? The majority of approaches that perform semantic labeling on numeric data in the literature treat numbers in numeric data the same way. In this research question, we ask whether we can get better performance applying different methods for the different types of numeric data ${ }^{3}$.

\section{Hypothesis}

We propose an automatic semantic labeling approach that exploits the richness of knowledge graphs to propose the semantic classes and properties of some given tabular data. This approach matches the input data to equivalent semantic classes and properties without depending on fixed data sources or search engines. It also does not require any prior profiling of the given knowledge graph or any manual tasks. This analysis translates into the following hypothesis: "the majority of subject and numeric columns in tabular data on the Web can be labeled automatically using knowledge graphs as the only external source of information."

\subsection{Methodology}

We reviewed semantic labeling approaches in the literature and observed several shortcomings such as the reliance on search engines and the need for manual intervention (Section 3.3). We gathered these shortcomings and hypothesized that we can overcome them (Section 4.1).

In this work, we used quantitative methods to evaluate our hypothesis. We designed multiple experiments to answer each of the research questions. We reported the

\footnotetext{
${ }^{2}$ They are ratio-and-interval-other and ratio-and-interval-counts, consecutively. This is discussed in more details in Section 6.2.

${ }^{3}$ We present different types of numeric data in Section 6.2.
} 
results of each experiment using precision, recall, and F1 scores. Such measurements are considered the de facto standard in evaluating and reporting semantic labeling results. Precision $[14,75,80,84,61,21,50,85,64,76,26,86,63]$, recall $[80,84,61$, $21,50,85,64,76,26,86,63]$ and $\mathrm{F} 1$ score $[45,80,61,21,50,85,64,76,26,86,63]$ are the most used measures to report the performance. Tonon et al. [77] use Mean Average Precision and Normalize Discounted Cumulative Gain as their aim is to measure the relevancy. Mean Reciprocal Rank is also used by some works $[62,56,76]$ which focus on the rank of the suggested labels. We explored the data used by the different approaches (Table 3.2), and found that the majority were either not published or not easy to use. We will present the data that we used in Section 4.3.

Here, we briefly describe the experiments to address the different research questions. To answer the first research question (Q.1), we designed an experiment to assign semantic classes from the knowledge graph to tables gathered from the Web. We checked how many of the assigned semantic classes were correct. We computed the precision, recall, and F1 score to answer the question. The details of the experiment are described in Chapter 5.

Similarly, for the second research question (Q.2) we built an experiment to assign semantic properties from the knowledge graph to a set of tables gathered from the Web. The correctness of the assigned properties is checked, and the precision, recall, and F1 score are computed to answer the research question. We described the details of the experiment in Section 6.1.

To address the final question (Q.3), we introduced a typology for numeric values for semantic labeling, which improves the performance of semantic labeling of numeric data. The experiment takes the numeric properties from the knowledge graph and automatically assigns the (numeric) types and sub-types. This is also done for numeric columns in the tabular data. The precision, recall, and F1 score are computed for the assignment for the numeric types and the numeric properties from the knowledge graph. The computed scores are used to answer the research question - whether taking into account the typology of numeric columns would improve semantic labeling. All the details of the experiment are explained in Section 6.2. 


\subsection{Data for Evaluation}

One of the crucial aspects of the experiments we performed is the data we used. It is essential to the evaluation of the hypothesis. Also, the use of such public data enables the comparison against the different approaches that use the same data. In total, we used three public datasets: Olympic Games 2020, T2Dv2, and T2DT. The first one is the only one we collected, and we made it public [4]. We used version one of this dataset. T2Dv2 is version 2 of T2D, an annotated subset of Web Data Commons. The owners made it public [63]. T2DT is an annotated subset of Web Data Commons which is also made public [26].

\subsubsection{Olympic Games 2020}

This dataset is the only one we collected, while the other two are published by other researchers. This is done in a systematic way to reduce possible bias. To collect the data about the Olympic Games of 2020 we visited the Wikipedia page for the list of sports ${ }^{4}$. For each sport in the list, we searched the Web using the Google search engine. For each sport, we only collect the first table we encounter. We inspect the results of the first two pages for each sport. We eliminate sports with no relevant data (e.g., stadium size). We also avoided any tabular data from Wikipedia to reduce the amount of bias as DBpedia has data extracted from Wikipedia. For the remaining sports, we inspect the English DBpedia ${ }^{5}$ for the same concepts (classes), and we discard sports that have no corresponding data in the English DBpedia. The remaining sports have corresponding data we gathered from the Web and from DBpedia. After that, we annotate the tables with classes and properties from the English DBpedia. The description of the dataset is shown in Table 4.1. We published the Olympic Games dataset, so it can be used by others to compare the performance of different semantic labeling approaches [4].

Preprocessing: We did a single data transformation, which is merging the columns

\footnotetext{
${ }^{4}$ https://en.wikipedia.org/wiki/2020_Summer_0lympics

${ }^{5}$ http://dbpedia.org/sparql
} 


\begin{tabular}{lc}
\hline Attribute & Value \\
\hline number of tables & 12 \\
number of subject columns & 12 \\
number of numeric columns & 24 \\
\hline
\end{tabular}

Table 4.1: Summary of the Olympic Games dataset

"FIRSTNAME" and "SECONDNAME" as our approach does not work if the entity column is separated into two columns. We performed that on the files themselves (we updated the files before feeding them to our application). Obviously, this may be easily automated in the future as an additional feature of our implementation.

\subsubsection{T2Dv2}

This dataset is a subset of Web Data Commons tables, which has been manually annotated with DBpedia classes and properties. T2Dv2 is slightly larger than the previous version (T2Dv1), and the tables are cleaner (e.g., they do not contain HTML tags). The subject columns of this dataset are also annotated with classes from DBpedia. For the numeric columns, we manually annotated them with properties from DBpedia. We were not able to annotate some of the subject columns as some were ambiguous and we were not able to understand them, and some have no corresponding properties in DBpedia. We describe the dataset in Table 4.2 and list the most common classes in Table 4.3.

\subsubsection{T2DT}

This dataset is a subset of the Web Data Commons, gathered by Ermilov et. al [26]. We are not aware of any other work that uses this dataset for semantic labeling. We refer to this dataset as T2DT. Although this dataset includes the identified subject columns, it does not include the classes (annotation) of the subject columns. The description of this dataset is shown in Table 4.4. 


\begin{tabular}{lc}
\hline Attribute & Value \\
\hline number of tables & 237 \\
number of subject columns & 237 \\
number of numeric columns & 319 \\
number of understood numeric columns & 232 \\
number of understood numeric columns found in DBpedia & 137 \\
\hline
\end{tabular}

Table 4.2: Summary of T2Dv2 dataset

\begin{tabular}{lc}
\hline Class & Number of tables \\
\hline Country & 33 \\
VideoGame & 19 \\
Company & 17 \\
Film & 17 \\
Mountain & 13 \\
\hline
\end{tabular}

Table 4.3: Most common classes in T2Dv2

\subsection{Assumptions}

In this section, we mention the assumptions that we consider in this work. They are crucial and removing any of these assumptions might invalidate the hypothesis of this work. We list the assumptions as follow:

A.1 There is a single subject column in each table. We do not take into account tables that have their subjects split into multiple columns (e.g., names split into two columns: first name and last name).

A.2 The knowledge graph can be queried with SPARQL.

A.3 Equivalent classes and properties of the columns in the tabular data (which are to be annotated) are declared in the knowledge graph.

A.4 For each subject column, there is only one correct corresponding semantic label 


\begin{tabular}{lc}
\hline Attribute & Value \\
\hline number of tables & 116 \\
number of subject columns & 112 \\
\hline
\end{tabular}

Table 4.4: Summary of T2DT dataset

from the knowledge graph.

A.5 The input tables are vertical where the header is the first row (if there is one). Nonetheless, this does not limit our approach as horizontal tables can be transposed easily, it is just a matter of detecting whether the table is vertical or horizontal.

A.6 The knowledge graph contains the ABox - instances or data following the adopted Ontology.

A.7 Exact match is sufficient to match the majority of entity names/labels in subject columns in tabular data with the equivalent entities (or entities with a similar name) in the knowledge graph ${ }^{6}$.

\subsection{Limitations}

In this section, we list the limitations of the proposed in this work. We focus on limitations related to the concepts and algorithms. We ignore limitations related to the implementation details (e.g., maximum number of rows and columns in the input files, the size limit of the knowledge graph, the longest path of the class hierarchy). Nonetheless, these reported limitations may be addressed in the future. The limitations of our work are:

L.1 Subjects that are split into multiple columns are not supported for the subject column semantic labeling. There are cases where the subject is presented by multiple columns, but they are rare [80].

\footnotetext{
${ }^{6}$ This assumption is based on the available gold standard (T2Dv2). Nonetheless, this might not be the case for other datasets. Also, this does not ensure a correct entity linking.
} 
L.2 Only Arabic numerals are supported for the semantic labeling of numeric columns.

L.3 Semantic labeling of numeric columns of complex (unreal) numbers is not supported.

L.4 Semantic labeling of numeric columns does not support numbers with bases other than ten. 


\section{Chapter 5}

\section{Semantic Labeling of Subject}

\section{Columns}

In this chapter, we propose an approach to assign semantic labels from a given knowledge graph to subjects columns in tabular data. The approach exploits the knowledge stored in the knowledge graph, and uses that knowledge to predict the class (also known as type) of the subject column. In this chapter, we use the terms class and type to refer to the same thing.

A subject column (also known as entity column [64]) of a table is a column which acts as the primary key of that table [86]. Subject columns hence contain the labels of the entities whose attributes are described in the rest of the columns of the table. Note that subject columns need not be unique $[80,86]^{1}$

First (Section 5.1), we present an initial approach where we score the classes in the whole subject column. This approach suffers from performance problems, therefore, we extended this approach to scale to larger files (Section 5.2) being able to score subsets of the subject column in a parallel fashion.

\footnotetext{
${ }^{1}$ In the work of Venetis et al. [80], the authors stress that subject columns need not be a key as they may contain duplicates, while others [63] refer to them as the key columns. However, they all agree that the subject column is the one that contains the entities of the main topic of the table.
} 


\subsection{An Initial Approach for Semantic Labeling of Subject Columns}

Our algorithm expects the subject column id with the table (as a file) and a SPARQL endpoint to be used as the source of knowledge ${ }^{2}$. The algorithm consists of the following steps: cells typing, class-graph construction, and class scoring. The first step assigns types to individual cells from the knowledge graph. In the class-graph construction step, the class-graph (type-graph) is built using rdfs:subClassOf. The last step scores each class in the graph and picks the potential classes (types). We start with a simplified working example to illustrate the approach and continue with the description of the algorithm and the scoring functions.

\subsubsection{A Working Example}

Let us consider the table shown in Fig. 5-1a, where we want to annotate a table that seems to contain people with the DBpedia knowledge graph. The entity column that we are interested in is the one with the "Player name" header. The first step of our approach is annotating each cell in the entity column, in our case, "Facundo Campazzo". We query DBpedia for an entity that has the name "Facundo Campazzo". We get the URI of the entity: http://dbpedia.org/resource/Facundo_Campazzo. Then, we query the knowledge graph for the classes of "Facundo Campazzo" and assign these types to the cell of "Facundo Campazzo" (Fig. 5-1b). We do the same for the other cells in the column. After that, we build the class-graph that contains all the types of the entities linked to the cells. In our example, we build a graph of the types of "Facundo Campazzo" (Fig. 5-1c). Note that in that graph, we have smaller circles that do not contain any text. We add them to illustrate that we may have other types (for other cells) that are not ancestors of basketball player. The last step

\footnotetext{
${ }^{2}$ Although the entity column may be identified by the user, our approach works without this assumption without any loss of generality i.e., it would work with entity columns in the middle or at the end of the data source, given that they are identified correctly. Furthermore, Cafarella et al. [14] found that the second most heavily weighted feature to search for subject columns in Web Tables is the number of hits in the left-most column.
} 
Listing 5.1: Get entities for a cell

select distinct ?subject

where\{?subject ?property "Facundo_Campazzo"@en \}

is to score the class-graph using Eq. (5.1), and pick the type with the highest score, which is basketball player.

\subsubsection{Algorithm Description}

We illustrate the workflow of our approach in Fig. 5-2. We approach the problem by first performing entity linking in the tabular datasets to the corresponding ones in the knowledge graph. As in Fig. 5-2 (step 1), the first step takes the input files (to be annotated) and a knowledge graph to annotate the cells. The next step is to build a class-graph of the types gathered in the first step. It takes as input the annotated cells and the knowledge graph to build a class-graph (hierarchy) from the types of the cells (step 2 in Fig. 5-2). The last step is to score each class in the class-graph using Eq. (5.1). The class with the highest score is picked to be the type of the entity column (step 3 in Fig. 5-2).

\subsubsection{Cells typing}

For each cell in the entity column in the input data, we assign a potential list of entities (in the form of URIs). We get the list of entities by querying the knowledge graph for entities having the cell's value as the object of some triples (Listing 5.1). In this paper, we use exact matching to get entities for a given cell. This may be extended in the future to other types of matching that are more flexible, although the obtained results suggest that this may not be necessary. After that, for each entity matched to a cell, we get the list of types for that entity (Listing 5.2), and the types of a single cell are the types of that cell's entities. 


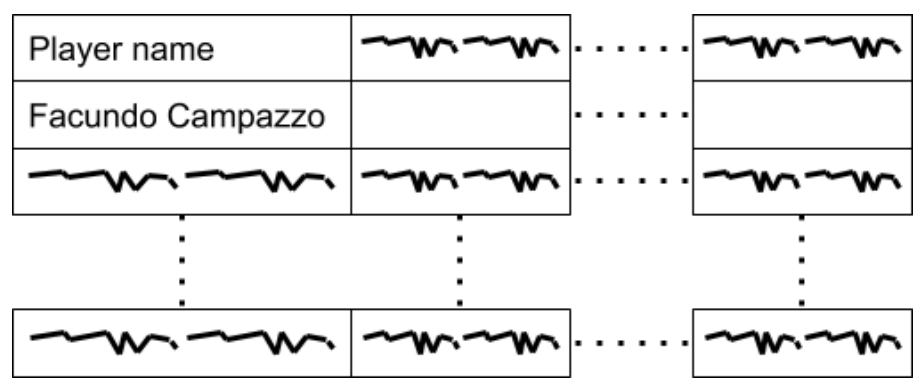

(a) Example of an input file

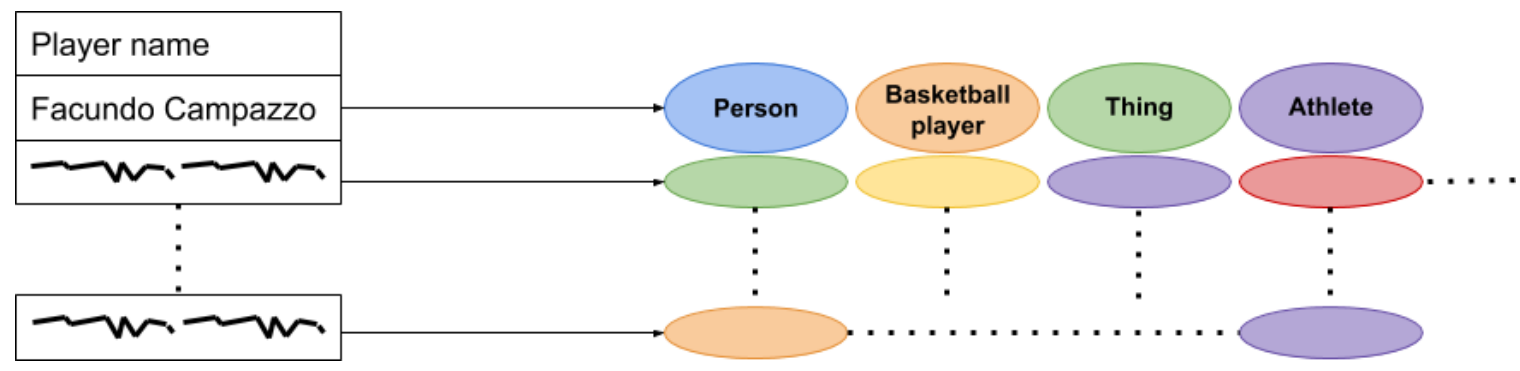

(b) Example of annotation for each cell

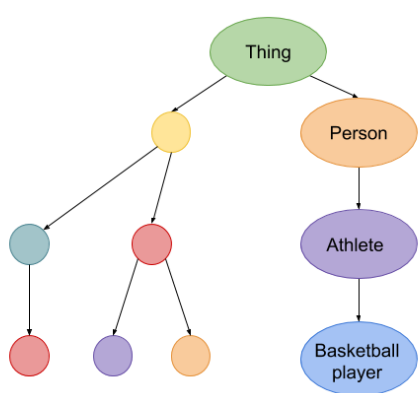

(c) Example of type graph

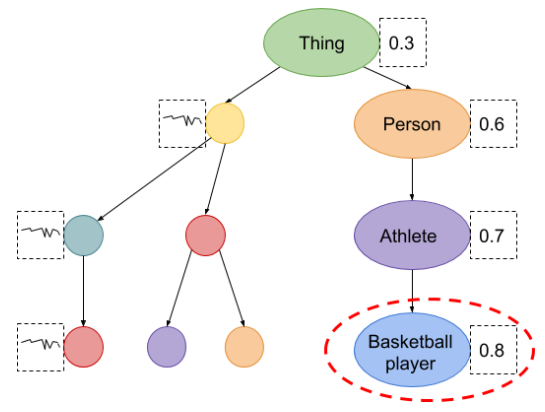

(d) Example of type scoring

Figure 5-1: Example of semantic annotation

Listing 5.2: Get classes to which an entity belongs

prefix dbr: $<$ http://dbpedia.org/resource/ $>$

select distinct ? class

where $\{\mathrm{dbr}$ :Facundo_Campazzo a ?class $\}$ 


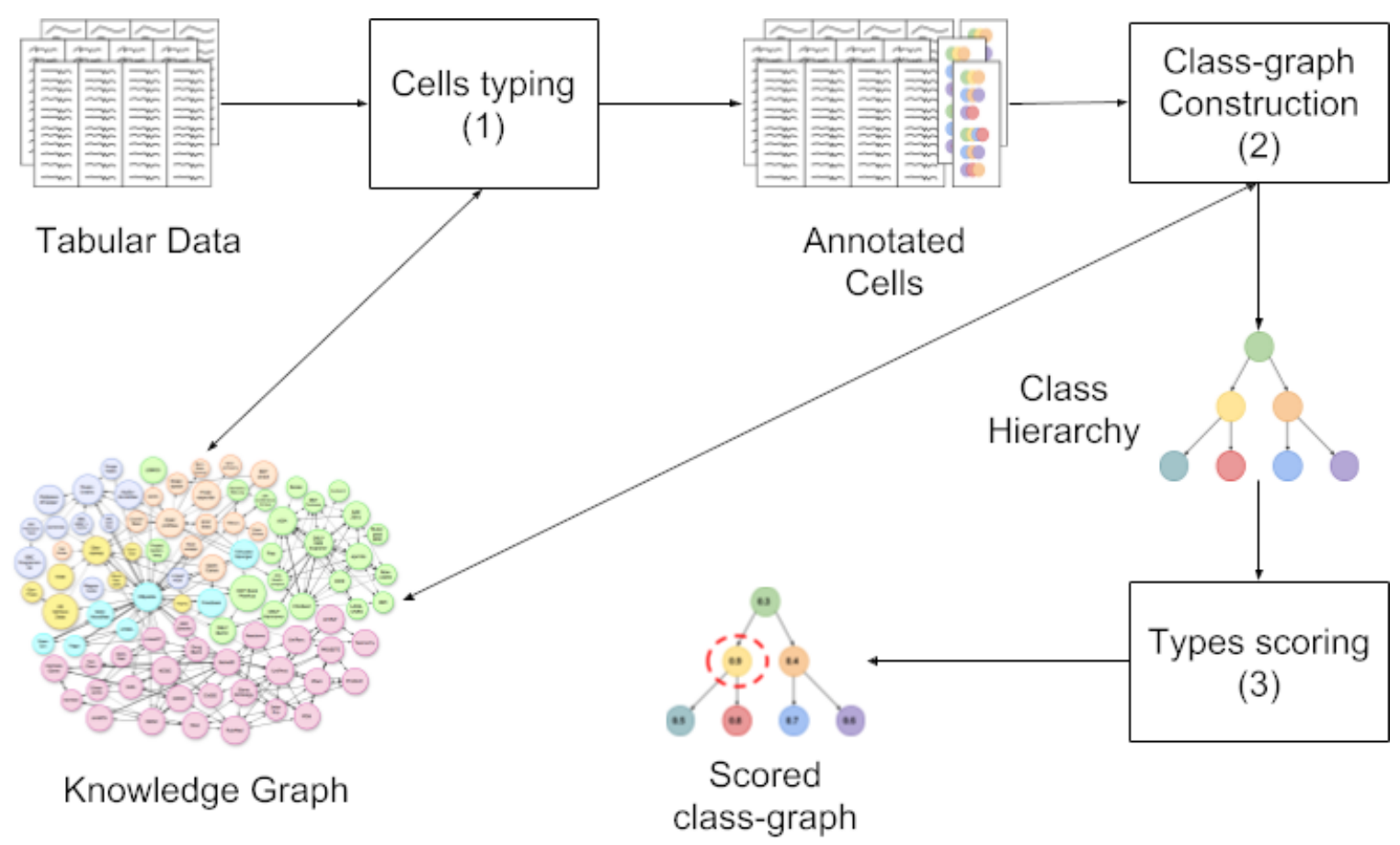

Figure 5-2: Semantic annotation workflow

Listing 5.3: Get parent classes for an entity

prefix dbo: $<$ http://dbpedia.org/ontology/>

select distinct ? parent

where $\{$ dbo: BasketballPlayer rdfs:subClassOf ?parent. $\}$

\subsubsection{Class-Graph Construction}

At this point, we have assigned a list of classes to each entity. Note that given our exact match restriction, we may not find classes for all. Nonetheless, we can still obtain good results independently of this.

We build the class graph by first gathering the list of classes for each cell for each entity. We query each class to get its parents (Listing 5.3). If a class has no parents, then it is a root; otherwise, the parents will also be queried for their parents if not already in the graph and will be linked accordingly. The resulting graph is referred to as class-graph (also known as type-graph, class-hierarchy, and type-hierarchy). 


\subsubsection{Class Scoring}

There are two contradicting preferences to choose the most suitable class in the classgraph: 1) prefer the most specific types; 2) prefer types that cover the majority of instances. In terms of the class-graph, the most specific classes tend to be in the leaves, while the nodes (classes) that cover the most are at the top of the graph. Typically, the root (e.g., owl:Thing) covers all the instances. We propose a formula to weight the two features and maximize the sum over the nodes in the graph, Equation (5.1). The coverage score of a type (node) $t$ is denoted by $f_{c}(t)$ and the specificity score by $f_{s}(t)$. Alpha $(\alpha)$ is used to weight the coverage score and the specificity score of a node $t$ (related proofs are in Section 5.1.3). The specificity score indicates how specific a type $t$ is, while the coverage score denotes how much a type $t$ covers. Equation (5.1) maximizes the score $f$ by choosing the type $t$ that maximizes the sum of the specificity score and the coverage score. We present the notation we use for the scoring functions in Table 5.1.

$$
\arg \max _{t} f(t)=\alpha * f_{c}(t)+(1-\alpha) * f_{s}(t)
$$

\subsubsection{Coverage}

The coverage score indicates how many instances a type $t$ covers. The higher the coverage score $f_{c}$ is, the more entities the type $t$ covers. The basic idea is to have for each cell a score of 1 . This cell score will be shared among the entities of that cell and will be divided evenly. The score of each entity will also be divided evenly among its types. This is shown in Equation (5.2), where $v$ is a text value in the entity column, and $e$ is an entity that has the label/name of $v . \Psi(t)$ represents the text values that has the type $t$ as one of their types/classes. The function $Z$ returns the entities of a given text value, and $Q$ returns the classes of any given entity. $\|Z(v)\|$ represents the number of entities from the knowledge graph that are linked to $v$ and $\|Q(e)\|$ denotes 
Table 5.1: Notation for scoring functions in Chapter 5

\begin{tabular}{cl}
\hline Notation & Meaning \\
\hline$\rho$ & the number of partitions of a column \\
$\Psi(t)$ & cells that have the type $t$ in a given column \\
$\Psi_{a}(t)$ & cells in a column $a$ that have the type $t$ \\
$\Psi_{a \varrho}(t)$ & cells in a partition $\varrho$ in a column $a$ that have the type $t$ \\
$L_{c}(t)$ & the local coverage score for a type $t$ \\
$L_{c_{\varrho}}(t)$ & the local coverage score for a type $t$ in partition $\varrho$ \\
$I_{c_{a}}(t)$ & the $I_{c}$ of a type $t$ of a column $a$ \\
$I_{c_{a}}(t)$ & the $I_{c}$ of a type $t$ of a partition $\varrho$ of a column $a$ \\
$U(t)$ & the children of type $t$ \\
$Z(v)$ & entities of a given text value $v$ \\
$\|Z(v)\|$ & the number of entities of a given text value $v$ \\
$Q(e)$ & the types of an entity $e$ \\
$\|Q(e)\|$ & the number of types of an entity $e$ \\
$R(t)$ & the entities of a type $t$ from the given knowledge graph \\
$\|R(t)\|$ & the number of entities of a type $t$ \\
$m$ & the number of annotated cells in a given column \\
$f_{c}(t)$ & coverage score of a semantic type $t$ \\
$f_{s}(t)$ & specificity score of a semantic type $t$ \\
$\alpha$ & balances specificity score $f_{s}(t)$ and coverage score $f_{c}(t)$ \\
$p r(t)$ & the parent of a type $t$
\end{tabular}

the number of classes for $e$ that are returned from the knowledge graph.

$$
I_{c}(t)=\sum_{v}^{\Psi(t)} \sum_{e}^{Z(v)} \frac{1}{\|Z(v)\| \times\|Q(e)\|} ; t \in Q(e)
$$

For the coverage, we also want a type $t$ to include the coverage of its children. We represent that in Equation (5.3), where $U(t)$ are the children of $t$. If $t$ does not have any child, its value will be $I_{c}(t)$.

$$
L_{c}(t)=I_{c}(t)+\sum_{u}^{U(t)} L_{c}(u)
$$

Since the value of $L_{c}$ will increase as the number of cells increases, we normalize the coverage score by dividing it by $m$, where $m$ is the number of cells that are 
matched to at least one entity, Equation (5.4).

$$
f_{c}(t)=\frac{L_{c}(t)}{m}
$$

\subsubsection{Specificity}

Specificity score indicates how specific/narrow a given type $t$ is. A high specificity score means a very specific type. We follow the intuition that a very narrow type, has a fewer number of entities compared to another type. For example, the number of physicists is much less than the number of scientists, and the number of scientists is much less than the number of humans. Saying that a person is a physicist is more specific than saying that this person is a scientist. Another way to look at that is if we picked a human randomly, the probability of this person being a physicist is lower than the probability of this person being a scientist. In accordance with information theory, as the probability decreases, the value of the corresponding piece of information increases.

Therefore, we divide the number of entities of a type $t$ which is $\|R(t)\|$ by the number of entities of its parent $(\|R(\operatorname{pr}(t))\|)$, where $\operatorname{pr}(t)$ is the parent with the highest number of entities, Equation (5.5). Note that the number of entities here is the number of entities returned from the knowledge graph. In case $t$ does not have parents, the value of $I_{s}(t)$ will be 1 .

$$
I_{s}(t)=\frac{\|R(t)\|}{\|R(p r(t))\|}
$$

The score $I_{s}$ is computed from the type $t$ to the root; this is done by multiplying $I_{s}$ for all nodes along the way as we show in Equation (5.6). In the case of multiple paths, the lower $L_{s}$ is chosen. In case a class $t$ has no parents, its $L_{s}$ value will be set to 1 .

$$
L_{s}(t)=I_{s}(t) \times L_{s}(p r(t)) .
$$

More specific types yield low $L_{s}$. However, we want to maximize both $f_{c}$ and $f_{s}$. 
We need a scoring function that increases as the value of $L_{s}$ decreases.

We present multiple formulas to compute the specificity of a given type $t$. All of them follow the intuition we mentioned earlier. Relevant proofs are provided in Section 5.1.3, and we show how we obtain the different functions in Section 5.1.4.

$$
\begin{gathered}
f_{s 1}(t)=\sqrt{\left(1-L_{s}(t)^{2}\right)} \\
f_{s 2}(t)=-L_{s}(t)^{2}+1 \\
f_{s 3}(t)=-L_{s}(t)+1 \\
f_{s 4}(t)=1-\sqrt{L_{s}(t)} \\
f_{s 5}(t)=\left(1-\sqrt{L_{s}(t)}\right)^{2}
\end{gathered}
$$

When we talk about the specificity function $f_{s}$, it may refer to any of the specificity functions mentioned above. In the evaluation section, we test all of them and compare the score for each. After computing the coverage score $f_{c}$ and the specificity score $f_{s}$ for each type, we compute the overall score $f$ for each class in the resulted class-graph following Equation 5.1. Finally, the types are ordered in decreasing order by the value of $f$, as the node with the highest score represents the type of the entity column; the other top types in the list are picked if the first one is rejected by the user.

\subsubsection{Scoring Functions Proofs}

In Section 5.1.2, we mention intuitions about the specificity and coverage functions. There are more entities of any parent class (e.g., athlete) than any of its children (sub-class) classes (e.g., basketball players). Also, knowing that an entity is of a more specific (lower in the sub-class hierarchy) is more valuable than knowing that it is 
of a more general type (higher in the sub-class hierarchy). For example, it is more interesting to know if someone is a basketball player than an athlete. We formulated these intuitions in Section 5.1.2, and we validate them in this Section. We prove that the coverage scoring function of a descendant of a type $t$ will never obtain a coverage score higher than any of its ancestors and that the specificity scoring function of a descendant of a type $t$ will never obtain a specificity score lower than any of its ancestors. For all the below proofs, we assume that the number of entities of any class is more than the number of entities of any of its descendants (sub-classes).

\subsubsection{Coverage}

Lemma 1. Given two types $t_{1}$ and $t_{2}$, where $t_{1}$ is an ancestor of $t_{2}$. The coverage score of $t_{1}$ is greater than the coverage score of $t_{2}$

$$
f_{c}\left(t_{1}\right)>f_{c}\left(t_{2}\right)
$$

Proof. To prove it by contradiction, let us assume that $f_{c}\left(t_{2}\right) \geq f_{c}\left(t_{1}\right)$

$$
\begin{gathered}
L_{c}\left(t_{2}\right) / m \geq L_{c}\left(t_{1}\right) / m \\
L_{c}\left(t_{2}\right) \geq L_{c}\left(t_{1}\right) \\
L_{c}\left(t_{2}\right) \geq I_{c}\left(t_{1}\right)+\sum_{u} L_{c}(u)
\end{gathered}
$$

since $t_{2}$ is a descendant of $t_{1}$, then $t_{2}$ is in $\sum_{u} L_{c}(u)$

$$
L_{c}\left(t_{2}\right) \geq I_{c}\left(t_{1}\right)+L_{c}\left(t_{2}\right)+\ldots
$$

since all the terms are positive, then this proposition is false.

Hence, lemma 1 is proved by contradiction 


\subsubsection{Specificity}

Lemma 2. Given two types $t_{1}$ and $t_{2}$, where $t_{1}$ is an ancestor of $t_{2}$. The specificity score $f_{s 1}$ of $t_{2}$ is greater than the specificity $f_{s 1}$ of $t_{1}$ i.e. $f_{s 1}\left(t_{2}\right)>f_{s 1}\left(t_{1}\right)$.

Proof. To prove that by contradiction,

we assume that: $f_{s 1}\left(t_{2}\right) \leq f_{s 1}\left(t_{1}\right)$

$$
\begin{aligned}
& \sqrt{1-L_{s}\left(t_{2}\right)^{2}} \leq \sqrt{1-L_{s}\left(t_{1}\right)^{2}} \\
& 1-L_{s}\left(t_{2}\right)^{2} \leq 1-L_{s}\left(t_{1}\right)^{2} \\
&-L_{s}\left(t_{2}\right)^{2} \leq-L_{s}\left(t_{1}\right)^{2} \\
& L_{s}\left(t_{2}\right)^{2} \geq L_{s}\left(t_{1}\right)^{2} \\
& L_{s}\left(t_{2}\right) \geq L_{s}\left(t_{1}\right) \\
& I_{s}\left(t_{2}\right) * \ldots * L_{s}\left(t_{1}\right) \geq L_{s}\left(t_{1}\right)
\end{aligned}
$$

Since all the terms (the $I_{s}$ 's and the $L_{s}$ 's ) are smaller than 1 and are positive, this is impossible. Hence, it is proven by contradiction

Lemma 3. Given two types $t_{1}$ and $t_{2}$, where $t_{1}$ is an ancestor of $t_{2}$. The specificity score $f_{s 2}$ of $t_{2}$ is greater than the specificity $f_{s 2}$ of $t_{1}$ i.e. $f_{s 2}\left(t_{2}\right)>f_{s 2}\left(t_{1}\right)$.

Proof. Let us assume that: $f_{s 2}\left(t_{2}\right) \leq f_{s 2}\left(t_{1}\right)$

$$
\begin{gathered}
-L_{s}\left(t_{2}\right)^{2}+1 \leq-L_{s}\left(t_{1}\right)^{2}+1 \\
-L_{s}\left(t_{2}\right)^{2} \leq-L_{s}\left(t_{1}\right)^{2} \\
L_{s}\left(t_{2}\right)^{2} \geq L_{s}\left(t_{1}\right)^{2} \\
L_{s}\left(t_{2}\right) \geq L_{s}\left(t_{1}\right) \\
I_{s}\left(t_{2}\right) * \ldots * L_{s}\left(t_{1}\right) \geq L_{s}\left(t_{1}\right)
\end{gathered}
$$


Since all the terms are less than 1, this is impossible, hence, this lemma is proved by contradiction.

Lemma 4. Given two types $t_{1}$ and $t_{2}$, where $t_{1}$ is an ancestor of $t_{2}$. The specificity score $f_{s 3}$ of $t_{2}$ is greater than the specificity $f_{s 3}$ of $t_{1}$ i.e. $f_{s 3}\left(t_{2}\right)>f_{s 3}\left(t_{1}\right)$.

Proof. To prove this by contradiction, we assume that: $f_{s 3}\left(t_{2}\right) \leq f_{s 3}\left(t_{1}\right)$

$$
\begin{gathered}
-L_{s}\left(t_{2}\right)+1 \leq-L_{s}\left(t_{1}\right)+1 \\
-L_{s}\left(t_{2}\right) \leq-L_{s}\left(t_{1}\right) \\
L_{s}\left(t_{2}\right) \geq L_{s}\left(t_{1}\right) \\
I_{s}\left(t_{2}\right) * \ldots * L_{s}\left(t_{1}\right) \geq L_{s}\left(t_{1}\right)
\end{gathered}
$$

Since all the terms are less than 1 and are positive, this cannot hold; hence it is proven by contradiction

Lemma 5. Given two types $t_{1}$ and $t_{2}$, where $t_{1}$ is an ancestor of $t_{2}$. The specificity score $f_{s 4}$ of $t_{2}$ is greater than the specificity $f_{s 4}$ of $t_{1}$ i.e. $f_{s 4}\left(t_{2}\right)>f_{s 4}\left(t_{1}\right)$.

Proof. To prove this lemma, let us assume that: $f_{s 4}\left(t_{2}\right) \leq f_{s 4}\left(t_{1}\right)$

$$
\begin{aligned}
& 1-\sqrt{L_{s}\left(t_{2}\right)} \leq 1-\sqrt{L_{s}\left(t_{1}\right)} \\
&-\sqrt{L_{s}\left(t_{2}\right)} \leq-\sqrt{L_{s}\left(t_{1}\right)} \\
& \sqrt{L_{s}\left(t_{2}\right)} \geq \sqrt{L_{s}\left(t_{1}\right)} \\
& L_{s}\left(t_{2}\right) \geq L_{s}\left(t_{1}\right) \\
& I_{s}\left(t_{2}\right) * \ldots * L_{s}\left(t_{1}\right) \geq L_{s}\left(t_{1}\right)
\end{aligned}
$$

Since all the terms are less than 1 and are positive, this cannot hold; hence it is proven by contradiction 
Lemma 6. Given two types $t_{1}$ and $t_{2}$, where $t_{1}$ is an ancestor of $t_{2}$. The specificity score $f_{s 5}$ of $t_{2}$ is greater than the specificity $f_{s 5}$ of $t_{1}$ i.e. $f_{s 5}\left(t_{2}\right) \geq f_{s 5}\left(t_{1}\right)$.

Proof. Let us assume that: $f_{s 5}\left(t_{2}\right) \leq f_{s 5}\left(t_{1}\right)$

$$
\begin{aligned}
&\left(1-{\sqrt{L_{s}\left(t_{2}\right)}}^{2}\right. \leq\left(1-\sqrt{L_{s}\left(t_{1}\right)}\right)^{2} \\
& 1-\sqrt{L_{s}\left(t_{2}\right)} \leq 1-\sqrt{L_{s}\left(t_{1}\right)} \\
&-\sqrt{L_{s}\left(t_{2}\right)} \leq-\sqrt{L_{s}\left(t_{1}\right)} \\
& \sqrt{L_{s}\left(t_{2}\right)} \geq \sqrt{L_{s}\left(t_{1}\right)} \\
& L_{s}\left(t_{2}\right) \geq L_{s}\left(t_{1}\right) \\
& I_{s}\left(t_{2}\right) * \ldots * L_{s}\left(t_{1}\right) \geq L_{s}\left(t_{1}\right)
\end{aligned}
$$

Since all the terms are less than 1 and are positive, this is impossible, and hence it is proved by contradiction

\subsubsection{Optimal $\alpha$}

In this section, we explore the possibility of an optimal $\alpha$ for a class hierarchy with a single correct type. We explore three cases: 1) $t_{1}$ is an ancestor of $t_{2}$ and $t_{2}$ is the correct type; 2) $t_{1}$ is an ancestor of $t_{2}$ and $t_{1}$ is the correct type; 3$) t_{1}$ and $t_{2}$ are not on the same path (none of them is an ancestor of the other).

Lemma 7. Given two types $t_{1}$ and $t_{2}$, where $t_{1}$ is an ancestor of $t_{2}$ and $t_{2}$ is the correct type. There exists a value $\alpha$ such that $f\left(t_{2}\right)>f\left(t_{1}\right)$ (referred to as a correct $\alpha)$.

Proof. Given that $t_{1}$ is an ancestor of $t_{2}$ then

$$
\begin{aligned}
& 1 \geq f_{c}\left(t_{1}\right)>f_{c}\left(t_{2}\right)>0 \Longrightarrow f_{c}\left(t_{1}\right)-f_{c}\left(t_{2}\right)=A: A \in(0,1) \\
& 1>f_{s}\left(t_{2}\right)>f_{s}\left(t_{1}\right)>0 \Longrightarrow f_{s}\left(t_{2}\right)-f_{s}\left(t_{1}\right)=B: B \in(0,1)
\end{aligned}
$$




$$
\begin{aligned}
& \text { If } \exists \alpha: f\left(t_{2}\right)-f\left(t_{1}\right)>0 \\
& {\left[\alpha * f_{c}\left(t_{2}\right)+(1-\alpha) * f_{s}\left(t_{2}\right)\right]-\left[\alpha * f_{c}\left(t_{1}\right)+(1-\alpha) * f_{s}\left(t_{1}\right)\right]>0} \\
& \alpha * f_{c}\left(t_{2}\right)+(1-\alpha) * f_{s}\left(t_{2}\right)-\alpha * f_{c}\left(t_{1}\right)-(1-\alpha) * f_{s}\left(t_{1}\right)>0 \\
& {\left[\alpha * f_{c}\left(t_{2}\right)-\alpha * f_{c}\left(t_{1}\right)\right]+\left[(1-\alpha) * f_{s}\left(t_{2}\right)-(1-\alpha) * f_{s}\left(t_{1}\right)\right]>0} \\
& -\alpha *\left[-f_{c}\left(t_{2}\right)+f_{c}\left(t_{1}\right)\right]+(1-\alpha) *\left[* f_{s}\left(t_{2}\right)-f_{s}\left(t_{1}\right)\right]>0 \\
& -\alpha * A+(1-\alpha) * B>0 \\
& (1-\alpha) * B>\alpha * A \\
& (1-\alpha)>\alpha * \frac{A}{B} \\
& \frac{(1-\alpha)}{\alpha}>\frac{A}{B} \\
& \frac{1}{\alpha}-\frac{\alpha}{\alpha}>\frac{A}{B} \\
& \frac{1}{\alpha}-1>\frac{A}{B} \\
& \frac{1}{\alpha}>\frac{A}{B}+1 \\
& \frac{1}{\alpha}>\frac{A}{B}+\frac{B}{B} \\
& \frac{1}{\alpha}>\frac{A+B}{B} \\
& \alpha<\frac{B}{A+B}
\end{aligned}
$$

Since $A \in(1,0)$ and $B \in(1,0)$ hence, exist at least one value $\alpha$ that satisfies that.

Remark. The proof of Lemma 7 shows a range of values for $\alpha$ which satisfied the condition $\alpha<\frac{B}{A+B}$ (e.g., $\left.\alpha=0\right)$. Note that other lemmas should also be taken into account when picking a value of $\alpha$. For example, let us assume that we have $t_{1}, t_{2}$, and $t_{3}$, such that $t_{2}$ is an ancestor of $t_{3}$ and $t_{1}$ is an ancestor of $t_{2}$. Let us also assume that $t_{2}$ is the correct type. Not all values of $\alpha$ that satisfy $\alpha<\frac{B}{A+B}$ would also satisfy 
$f\left(t_{2}\right)>f\left(t_{3}\right)$ (e.g., $\left.\alpha=0\right)$. So, we would want $\alpha$ to yield $f\left(t_{2}\right)>f\left(t_{3}\right)$ as well as $\alpha<\frac{B}{A+B}$.

Lemma 8. Given two types $t_{1}$ and $t_{2}$, where $t_{1}$ is an ancestor of $t_{2}$ and $t_{1}$ is the correct type. There exists a value $\alpha$ such that $f\left(t_{1}\right)>f\left(t_{2}\right)$ (referred to as a correct $\alpha)$.

Proof.

$$
\begin{aligned}
& f_{c}\left(t_{1}\right)>f_{c}\left(t_{2}\right) \\
& f_{c}\left(t_{1}\right)-f_{c}\left(t_{2}\right)=A \\
& f_{s}\left(t_{2}\right)>f_{s}\left(t_{1}\right) \\
& f_{s}\left(t_{2}\right)-f_{s}\left(t_{1}\right)=B \\
& \text { If } \exists \alpha: f\left(t_{1}\right)>f\left(t_{2}\right) \\
& f\left(t_{1}\right)-f\left(t_{2}\right)>0 \\
& \alpha * f_{c}\left(t_{1}\right)+(1-\alpha) * f_{s}\left(t_{1}\right)-\alpha * f_{c}\left(t_{2}\right)-(1-\alpha) * f_{s}\left(t_{2}\right)>0 \\
& {\left[\alpha * f_{c}\left(t_{1}\right)-\alpha * f_{c}\left(t_{2}\right)\right]+\left[(1-\alpha) * f_{s}\left(t_{1}\right)-(1-\alpha) * f_{s}\left(t_{2}\right)\right]>0} \\
& \alpha *\left[f_{c}\left(t_{1}\right)-f_{c}\left(t_{2}\right)\right]-(1-\alpha) *\left[f_{s}\left(t_{2}\right)-f_{s}\left(t_{1}\right)\right]>0 \\
& \alpha * A-(1-\alpha) * B>0 \\
& \frac{\alpha}{(1-\alpha)}>\frac{B}{A} \\
& \frac{(1-\alpha)}{\alpha}<\frac{A}{B} \\
& \frac{1}{\alpha}-1<\frac{A}{B} \\
& \frac{1}{\alpha}<\frac{A+B}{B} \\
& \alpha>\frac{B}{A+B}
\end{aligned}
$$

since $A \in(0,1)$ and $B \in(0,1)$, there exists an $\alpha$ that satisfies that, hence this lemma 
is proved

Lemma 9. Given two types $t_{1}$ and $t_{2}$, where none of them is an ancestor of the other and $t_{1}$ is the correct type. There exists a value $\alpha$ such that $f\left(t_{1}\right)>f\left(t_{2}\right)$.

Proof. Case 1: $f_{c}\left(t_{1}\right)>f_{c}\left(t_{2}\right)$ and $f_{s}\left(t_{1}\right)>f_{s}\left(f_{2}\right)$

to prove this, we need this to hold: $f\left(t_{1}\right)>f\left(t_{2}\right)$

$$
\begin{gathered}
f\left(t_{1}\right)-f\left(t_{2}\right)>0 \\
\alpha f_{c}\left(t_{1}\right)+(1-\alpha) f_{s}\left(t_{1}\right)-\alpha f_{c}\left(t_{2}\right)-(1-\alpha) f_{s}\left(t_{2}\right)>0 \\
\alpha\left[f_{c}\left(t_{1}\right)-f_{c}\left(t_{2}\right)\right]+(1-\alpha)\left[f_{s}\left(t_{1}\right)-f_{s}\left(t_{2}\right)\right]>0 \\
\alpha\left[f_{c}\left(t_{1}\right)-f_{c}\left(t_{2}\right)\right]+(1-\alpha)\left[f_{s}\left(t_{1}\right)-f_{s}\left(t_{2}\right)\right]>0
\end{gathered}
$$

Which holds because $f_{c}\left(t_{1}\right)-f_{c}\left(t_{2}\right)>0$ and $f_{s}\left(t_{1}\right)-f_{s}\left(t_{2}\right)>0$

Case 2: $f_{c}\left(t_{1}\right)>f_{c}\left(t_{2}\right)$ and $f_{s}\left(t_{1}\right) \leq f_{s}\left(t_{2}\right)$

$$
\begin{gathered}
f\left(t_{1}\right)-f\left(t_{2}\right)>0 \\
\alpha f_{c}\left(t_{1}\right)+(1-\alpha) f_{s}\left(t_{1}\right)-\alpha f_{c}\left(t_{2}\right)-(1-\alpha) f_{s}\left(t_{2}\right)>0 \\
\alpha\left[f_{c}\left(t_{1}\right)-f_{c}\left(t_{2}\right)\right]+(1-\alpha)\left[f_{s}\left(t_{1}\right)-f_{s}\left(t_{2}\right)\right]>0 \\
\alpha\left[f_{c}\left(t_{1}\right)-f_{c}\left(t_{2}\right)\right]+(1-\alpha)\left[f_{s}\left(t_{1}\right)-f_{s}\left(t_{2}\right)\right]>0
\end{gathered}
$$

Since $f_{c}\left(t_{1}\right)-f_{c}\left(t_{2}\right)>0$ and $f_{s}\left(t_{1}\right)-f_{s}\left(t_{2}\right) \leq 0$, and $f_{c}$ and $f_{s}$ are bound between 0 and 1 , there exists at least one value alpha between 0 and 1 that satisfies this

Case 3: $f_{c}\left(t_{1}\right) \leq f_{c}\left(t_{2}\right)$ and $f_{s}\left(t_{1}\right)>f_{s}\left(f_{2}\right)$

$$
\begin{gathered}
f\left(t_{1}\right)-f\left(t_{2}\right)>0 \\
\alpha f_{c}\left(t_{1}\right)+(1-\alpha) f_{s}\left(t_{1}\right)-\alpha f_{c}\left(t_{2}\right)-(1-\alpha) f_{s}\left(t_{2}\right)>0
\end{gathered}
$$




$$
\begin{aligned}
& \alpha\left[f_{c}\left(t_{1}\right)-f_{c}\left(t_{2}\right)\right]+(1-\alpha)\left[f_{s}\left(t_{1}\right)-f_{s}\left(t_{2}\right)\right]>0 \\
& \alpha\left[f_{c}\left(t_{1}\right)-f_{c}\left(t_{2}\right)\right]+(1-\alpha)\left[f_{s}\left(t_{1}\right)-f_{s}\left(t_{2}\right)\right]>0
\end{aligned}
$$

Since $f_{c}\left(t_{1}\right)-f_{c}\left(t_{2}\right) \leq 0$ and $f_{s}\left(t_{1}\right)-f_{s}\left(t_{2}\right)>0$, and $f_{c}$ and $f_{s}$ are bound between 0 and 1 , there exists at least one value alpha between 0 and 1 that satisfies this

Case 4: $f_{c}\left(t_{1}\right) \leq f_{c}\left(t_{2}\right)$ and $f_{s}\left(t_{1}\right) \leq f_{s}\left(f_{2}\right)$

Similar to case 1 , this is impossible to hold for any $\alpha$ such that $1 \geq$ alpha $\geq 0$. This implies that $t_{1}$ is probably not the correct type as there are more entities classified as $t_{2}$ that are even more specific than $t_{1}$. More specifically, it means that most cell annotations are pointing towards $t_{2}$; which is not due to a typical mismatch, as typical mismatches do not converge to a high specificity type.

\subsubsection{Scoring Functions Construction}

In this section, we explain how we came up with each of the scoring functions. The ultimate goal is to have a type for the entity column. Our intuition is to choose the type that is correct for all the cells in the target column that we want to type. Each entity is annotated with one or more types. Given these types, we can construct the type hierarchy from the knowledge graph. We thought of choosing the most common type to cover all the cells. For example, given football players and basketball players, the most common thing is that they are all athletes. But in practice, it is challenging to link to the correct entities and to type them correctly from the cells $[82,69]$. If we are using DBpedia for example and we got one incorrect type for a cell, we can easily end up in the root of the type hierarchy ("owl:Thing" for DBpedia) as the common type.

We notice further that we need the type also to be as specific as possible. For a column of scientists names, it is more valuable for us to annotate them with the type "scientist" than "person" or "thing". It is also the case that the probability of having a column to have a more specific type (e.g., "scientist") is lower than having the type 
of the column to be of a more general type (e.g., "person"). Following information theory principles, the value of a piece of information increases as the probability of appearing decreases.

Following the above intuitions, we aim to have a type that covers most of the cells in the column and is also as specific as possible. These two goals pull in different directions: to increase the coverage (to cover as many cells as possible) pulls the type upwards (towards the root) and the specificity (to be as specific as possible in the types) pulls the type downwards (here we are picturing the type hierarchy to have the root on the top and the leaves on the bottom of the tree).

Our idea is to find a balance between the two to maximize the score. So, we have this simple function to balance the coverage with $\alpha$ and the specificity with $1-\alpha$. We formalized it here as follows:

$$
\arg \max _{t} f(t)=\alpha * f_{c}(t)+(1-\alpha) * f_{s}(t)
$$

Where $f_{c}$ is the coverage function, and $f_{s}$ is the specificity function. We explain the details for the coverage and specificity functions in the following sections.

\subsubsection{Coverage}

We are trying to construct a function that when maximized, picks the type that covers most of the cells. The first thing that we might think of is to use the type that is most common (often referred to as "majority"). But it only works if the types of each cell are almost the same, for example, if the majority of the cells has the type "footballPlayer". It won't work in the case of mixed types (e.g., "footballPlayer" and "basketballPlayer"), which should result in the type "athlete" instead.

Another way we thought of is to have all the types in the path in the type hierarchy from the type of the cell (e.g., "footballPlayer") to the root ("Thing" in the case of DBpedia). This way we have more general types (e.g., "athlete", "person"). We can have something like the majority but for each type in the hierarchy of each typed cell. In other words, the majority for each type in the path to the root. 
Uncertainty: Another intuition we can think of is related to uncertainty. A cell can have multiple types due to common names. An example of this is "Scott Arnold," there are multiple players with the same name. In such cases, we assign lower confidence to the types of such cells. We formulate it in a way such that the total value decreases as the number of types increases. Actually, each cell does not just get typed based on its value; we need to get the entities that have the name in the cell. For a given cell, we fetch the entities and then get the types for each of these entities. Each cell will have the types for each entity linked to that cell. We formulate the score as:

$$
\frac{1}{\|W(v)\|}
$$

The number of aggregated types for the cell value $v$ is denoted as $\|W(v)\|$.

Proportional Influence Entities with a lot of types have very little influence (if any) on the overall coverage score. For example, if we have the polymath "Bertrand Russell," he will be annotated with multiple types: logician, mathematician, historian, writer, and Nobel prize Laureate. Having multiple types, we have less confidence in the intended one for the context. If the input data is about Nobel prize Laureates, then this is the anticipated type. If the other people in the input data are mathematicians, then probably the type "mathematician" is the one that we are looking for. Having multiple types reduces confidence, and we reflect this on the formulation. We have this for each entity proposed for each cell so that entities with fewer types have higher confidence than the ones with more types. To formulate this, we first divide the score of a single cell for each entity. The intuition is that cells with more candidate entities have lower confidence. We will have $\frac{1}{\|Z(v)\|}$, and then for each entity $e$, we will have $\frac{1}{\|Q(e)\|}$ where $\|Z(v)\|$ is the number of entities for the cell value $v$ and $\|Q(e)\|$ is the number of types for the entity $e$. Combining these two, we present the equation $(E(v)$ are the entities for a given cell value $v)$ :

$$
\sum_{e} \frac{1}{\|Z(v)\|\|Q(e)\|}=1 \quad \forall e \in E(v)
$$

We illustrate this in Figure 5-3. Since we want to choose the type to maximize 


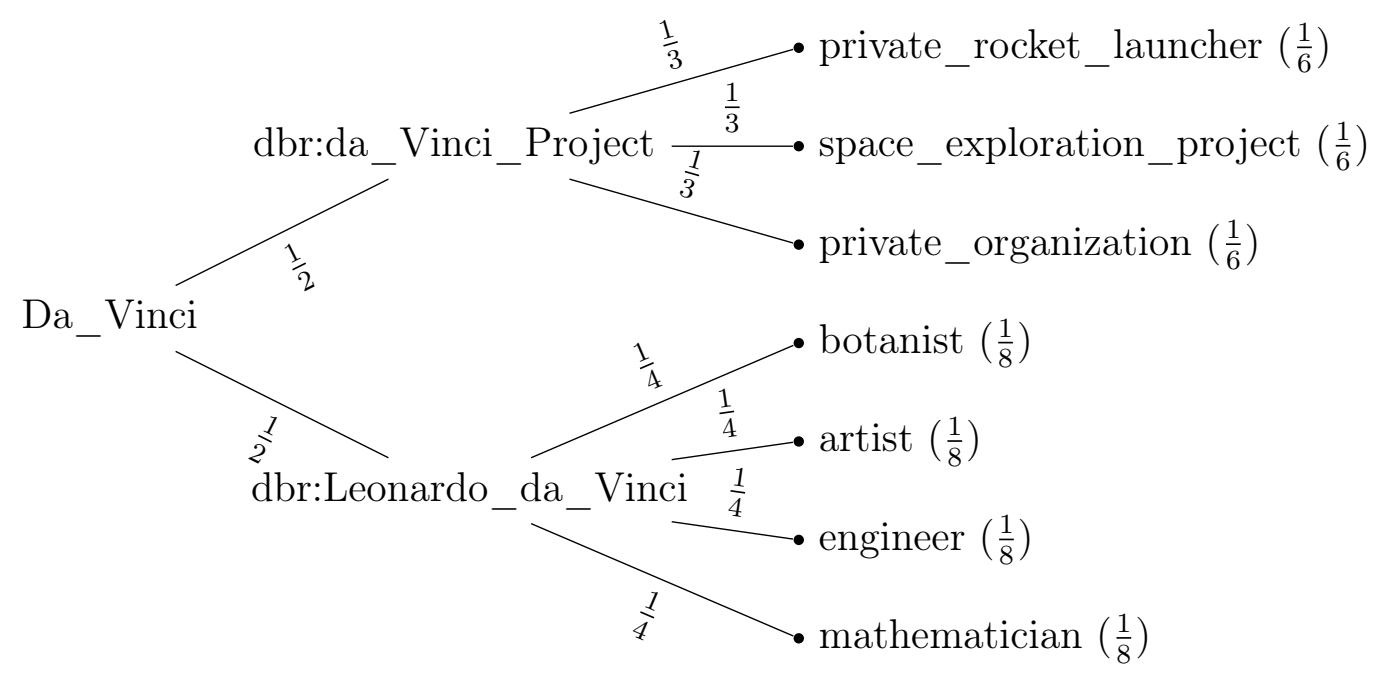

Figure 5-3: The coverage score break down for a single cell

the coverage score, we aggregate the coverage score for each type $t$ as follows:

$$
I_{c}(t)=\sum_{v} \sum_{e} \frac{1}{\|Z(v)\|\|Q(e)\|} \quad \forall v, e: t \in Q(e), e \in Z(e)
$$

Note that the $t$ in the equation is for the cells with a value $v$ that has an entity $e$ that has a type $t$. $I_{c}(t)$ is the coverage for a single type $t$.

Inclusion: In the previous equation, we did not take into account that a parent type (in the type hierarchy) actually covers all the cells its children cover. We include this in the recursive equation below:

$$
L_{c}(t)=I_{c}(t)+\sum_{u} L_{c}(u)
$$

So, the $L_{c}(t)$ coverage of a type $t$ is the $I_{c}(t)$ of $t$ plus the coverage $L_{c}$ of its children.

The $L_{c}(t)$ coverage increases as the number of entities increase. To overcome this, we normalize $L_{c}(t)$ by dividing it by the number of cells $m$. This would make the coverage insensitive to the number of cells in the column. The final coverage score would be: 


$$
f_{c}(t)=\frac{L_{c}(t)}{m}
$$

\subsubsection{Specificity}

Besides, choosing a type that covers as much from the cells as possible, we also want to be as specific as possible. More specific types are more valuable, as the probability decreases the value of the corresponding piece of information increases. This also follows our intuition that we are generally more interested in knowing that a given entity is a basketball player than it being an athlete or a person.

The first intuition that came to our mind is the level of the type in the type hierarchy. The deeper the type node is, the more specific it is. Even though the depth gives us an idea of the specificity of the type, it treats all levels the same way. Knowledge graphs may have more levels (subclass relation) in some domains (in the same knowledge graph) than others, which does not necessarily reflect the specificity. As an alternative, we thought of using the number of instances. To know how specific a type $t$ is, we divide the number of instances of a type $t(\|R(t)\|)$ by the number of instances of its parent $(\|R(\operatorname{pr}(t))\|)$ :

$$
I_{s}(t)=\frac{\|R(t)\|}{\|R(p r(t))\|}
$$

We refer to $I_{s}$ as the instance specificity ${ }^{3}$.

This gives us satisfactory results, but it only takes into account the type and its parent. Since the number of entities of a type is less than or equal to the number of entities of its parent, the results will be bounded by 0 and 1 . To include the specificity of its parent, we multiply the instance specificity of the type $t\left(I_{s}(t)\right)$ by the local specificity of its parent $\operatorname{pr}(t)\left(L_{s}(\operatorname{pr}(t))\right)$. The local specificity is computed as:

$$
L_{s}(t)=I_{s}(t) * L_{s}(\operatorname{pr}(t))
$$

\footnotetext{
${ }^{3}$ note that instance specificity does not refer to the specificity of an entity. It refers to the specificity of a single type $t$ in relation with its parent $\operatorname{pr}(t)$
} 


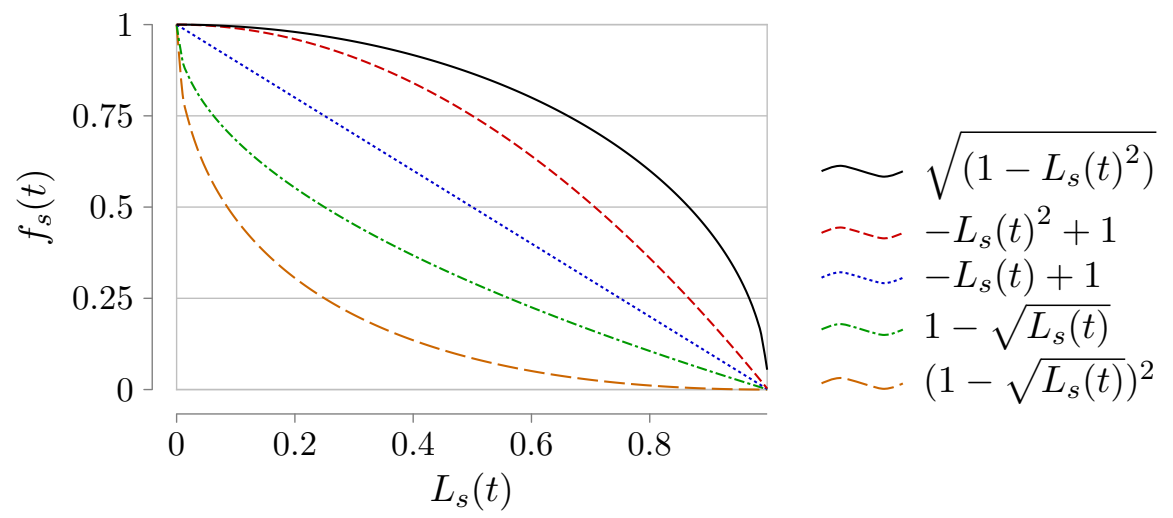

Figure 5-4: Different candidate specificity functions

Following the local specificity equation, the more specific the type $t$ is, the lower its value becomes. We are looking for a formula $f_{s}$ that increases as the local specificity decreases. The first thing that came to our mind is an inverted version of the square function, which is a curve. We can also experiment with a straight line as well. Another aspect is that we need is for the function to be bound by 0 and 1 . We pick five functions that satisfy these conditions: $\sqrt{\left(1-L_{s}(t)^{2}\right)},-L_{s}(t)^{2}+1,1-\sqrt{L_{s}(t)}$, $\left(1-\sqrt{L_{s}(t)}\right)^{2}$, and $-L_{s}(t)+1($ Fig 5-4).

\subsubsection{Experiment Design}

In this section, we describe our experimental evaluation to answer the research question Q.1, whether we can assign classes to subject columns automatically using a given knowledge graph, without any other external source of knowledge (e.g., linguistic resources).

In Section 5.1.3, we prove the existence of a value $\alpha$, i.e., Equation (5.1) results in a correct type. A correct type $t$ will have the highest score among the rest of the types for the entities linked to the cells in the entity column of a given CSV file (see Section 5.1.3.3). We also prove that the coverage score of a parent's node has a higher coverage score than any of its children (Section 5.1.3.1). In the same way, we prove that a child's node (type) has a higher specificity score than its parent (Section 5.1.3.2).

We built a Web application [5] (V2.0) to semantically annotate subject columns 
in tabular datasets automatically using a knowledge graph. Our application takes as an input the URL of the SPARQL endpoint and the input files to be annotated. It semantically annotates the entity columns and shows the potential types as a list of classes ordered descendingly from the highest score to the lowest score.

We experiment with two datasets: Olympic Games [4] and Web Data Commons version 2 (T2Dv2) [65], both of which were described in Section 4.3. Thanks to those experiments, we also show that our approach works with realistic datasets.

In all of our experiments, we use the English DBpedia because the data in T2Dv2 are annotated with the DBpedia classes. This permits us to compare the results of our approach with the gold standard of T2Dv2. Since DBpedia has classes imported from other ontologies (e.g., YAGO), we ignore such classes in the experiment. We report scores for each dataset, and we calculate the accuracy with different specificity functions.

\subsubsection{Experiment Results and Discussion}

We apply the approach with the Olympic Games and T2Dv2 datasets and report the precision, recall and, F1 scores. In Table 5.2, we report the scores for semantic annotation of the subject columns in the Olympic Games dataset. We got perfect precision and recall. We have a proof of the scoring functions that we use in Section 5.1.3. Our approach reaches a perfect score, given the fact that these data follow our intuitions, as we mention in Section 5.1.3. Also, we do not expect to have many cases where an incorrect type is more prominent than the correct type given that the difference in the depth is close (if not, it can be manipulated with the $\alpha$ value to give more weight to the coverage $f_{c}$ or specificity $f_{s}$ ). Since this is the case for the Olympic Games dataset, achieving a perfect score was not surprising.

T2Dv2 dataset is manually annotated with DBpedia classes. For a given file, if no annotation matches the class that is given in the gold standard, we consider it as a wrong annotation, even though some of them look correct or acceptable to us (e.g., typically people do not use AdministrativeRegion, but they use Place or City instead). We use a testing script to verify whether the annotation generated by our 
Table 5.2: Semantic-labeling scores for Olympic Games with different specificity functions (and the same coverage function)

\begin{tabular}{lcrc}
\hline$f_{s}$ & Precision & Recall & F1 \\
\hline$f_{s 1}$ & 1.0 & 1.0 & 1.0 \\
$f_{s 2}$ & 1.0 & 1.0 & 1.0 \\
$f_{s 3}$ & 1.0 & 1.0 & 1.0 \\
$f_{s 4}$ & 1.0 & 1.0 & 1.0 \\
$f_{s 5}$ & 1.0 & 1.0 & 1.0 \\
\hline \multicolumn{4}{r}{} \\
$\left.f_{s 1}=\sqrt{\left(1-L_{s}(t)^{2}\right.}\right)$ \\
$f_{s 2}=-L_{s}(t)^{2}+1$ \\
$f_{s 3}=-L_{s}(t)+1$ \\
$f_{s 4}=1-\sqrt{L_{s}(t)}$ \\
$f_{s 5}=\left(1-\sqrt{L_{s}(t)}\right)^{2}$ \\
\end{tabular}

application is the same or different from the one in the gold standard (T2Dv2). Since different tables can have different values of $\alpha$ that are optimal (which is also reported by Ritze et al. [63] that a single set of weights might not be the best for all tables ${ }^{4}$ ), the testing script tries with different values of $\alpha^{5}$.

We compare the annotations produced by our approach with the annotations reported in T2Dv2. We compare the performance of our approach with the results reported by Ritze et al. [63] (referred to as T2K Extended). They also show different baselines: Majority (how often the classes occur), Majority+ Frequency (taking into account the specificity ${ }^{6}$ ), Page attributes (e.g., Page title, URL, ...), Text (abstracts belongs to the classes), Majority + Frequency + Page attributes + Text $^{7}$. We report the results in Table 5.3.

We see that the best precision of our approach is 0.91 , which is slightly lower

\footnotetext{
${ }^{4}$ Note that our approach does not use the same features or weights.

${ }^{5} \alpha \in[0.45,0.4,0.35,0.3,0.25,0.2,0.1,0.05,0.01,0.005,0.001,0.0005,0.0001,0.00005,0.00001]$. This turns out to be a good set of values for $\alpha$ to balance coverage and specificity.

${ }^{6}$ It is different specificity function than the one we use in our approach.

${ }^{7}$ For more details on the baselines refer to the original paper by Ritze et al. [63].
} 
Table 5.3: Scores of semantic labeling of subject columns in T2Dv2

\begin{tabular}{lccc}
\hline Approach & Precision & Recall & F1 \\
\hline T2K (Majority) & 0.47 & 0.51 & 0.49 \\
T2K (Majority + Frequency) & 0.87 & 0.90 & 0.89 \\
T2K (Page attributes) & 0.97 & 0.37 & 0.53 \\
T2K (Text) & 0.75 & 0.34 & 0.46 \\
T2K (Majority + Frequency & 0.90 & 0.86 & 0.88 \\
Page attributes + Text) & & & \\
T2K Extended & 0.93 & 0.91 & $\mathbf{0 . 9 2}$ \\
\hline TADA-Entity $\left(f_{s 1}\right)$ & 0.68 & 0.96 & 0.79 \\
TADA-Entity $\left(f_{s 2}\right)$ & 0.75 & 0.96 & 0.84 \\
TADA-Entity $\left(f_{s 3}\right)$ & 0.91 & 0.97 & $\mathbf{0 . 9 4}$ \\
TADA-Entity $\left(f_{s 4}\right)$ & 0.85 & 0.97 & 0.90 \\
TADA-Entity $\left(f_{s 5}\right)$ & 0.84 & 0.97 & 0.90 \\
\hline & & & \\
$f_{s 1}=\sqrt{\left(1-L_{s}(t)^{2}\right)}$ & & & \\
$f_{s 2}=-L_{s}(t)^{2}+1$ & & & \\
$f_{s 3}=-L_{s}(t)+1$ & & & \\
$f_{s 4}=1-\sqrt{L_{s}(t)}$ & & & \\
$f_{s 5}=\left(1-\sqrt{L_{s}(t)}\right)^{2}$ & & & \\
& & & \\
\hline
\end{tabular}

than the T2K Extended approach. The precision of T2K (Page attributes) is very high (0.97), but covers only a small set as it has a low recall (0.37). The recall of our approach is 0.97, which is higher than T2K (0.91) and all the other baselines reported. The F1 of our approach (0.94) is higher than all the T2K approaches.

One of the main reasons that affect the precision score is the wrong entity linking, which is due to the use of naive entity linking. For example, many companies are named after their creator. An example of that is the famous Jewellery company "Cartier", which is named after its founder Louis-François Cartier. Another reason is missing data from the knowledge graph. This was the case for lakes labels, which are 
linked to boxers, sports teams, and places that share the same name. For example, one of them has the name "Molina", which is linked to a city in Chile, a soccer club "CF Molina", a cyclist "Juan Molina", an artist "Ralph Molina", but not to a Lake labeled "Molina". We did not take into account that famous labels are more prone to be wrongly annotated if only labels are taken into account. For example, the label "Lyon", which is a city in France, also has as candidate entities: an American footballer, a New Zealand music artist, and a Scottish politician. It could be intuitive to take into account what people think when the word "Lyon" is first introduced to them. However, that also means that facts or labels not commonly known will be more prone to be misclassified (given that a common fact about an entity with the same label exists).

\subsection{A Scalable Approach for Semantic Labeling of Subject Columns}

In this section, we describe the extension of our approach to allow for a scalable matching of subject columns to classes (labels) from a given knowledge graph. We present two approaches, one to detect subject columns, and the other to assign semantic classes from the knowledge graph to the subject columns.

There are two extremes to handling large amounts of data: having a large number of small data items or a small number of large data items. The former can be handled by queuing the files or distributing the files to multiple processes/servers performing the same tasks on different files. However, handling large data items is more tedious, as large files often do not fit in the main memory. In our approach, we address this by splitting large files into smaller parts, processing the splits separately (multiple instances), and then merging the intermediate results to reach the ultimate goal, extending the classical MapReduce framework [19, 20].

MapReduce is a framework introduced by Dean and Ghemawat, which is used to process a huge amount of data in a parallel fashion [19, 20]. According to Dean 
and Ghemawat [20], Google executes hundreds of thousand MapReduce jobs on their clusters every day, processing more than twenty petabytes per day. It is composed of two user-written functions: Map and Reduce. Typically, there are multiple instances of the same Map and Reduce functions. The Map function takes an input pair and produces intermediate results in the form of key/value pairs. Generally, there is a large collection of data, and there is a program that splits the data into smaller pieces, and each pair is fed to a Map instance. The Reduce function takes as input a key and its value(s) and produces an output, which is a result of a user-defined merge (e.g., count).

Challenge: making our initial approach scalable is not straight forward. Using MapReduce to split the data is easy, but performing semantic labeling without any loss in accuracy (precision and recall) is the challenging part. Using the scoring functions as is does not yield the same results compared to the initial approach. We show how this is done in Section 5.2.2 and we prove that both approaches are equivalent such that suggested labels will always be the same in Section 5.2.5.

In our approaches (detection and labeling), we follow the same general idea, but the types of intermediate values are different. Next, we explain the details of our approaches.

\subsubsection{Subject Column Detection Algorithm}

The first task is to spot the subject column for each table. As reported in [14, 80], subject columns tend to be on the left-most part of the tables. Starting from the leftmost, skipping columns that contain numbers tends to be a good approximation [80]. Another approximation presented by Ritze et al. [64] is based on the uniqueness of the values in a column. Their approach picks the columns with the most distinct values. It also favors the left-most in case of a tie. We experiment with the different approaches, adjusting them to make them scalable. Our subject column detection method is composed of three tasks: data splitting, subject column spotting, and subject column election. We show the overall workflow in Figure 5-5. 


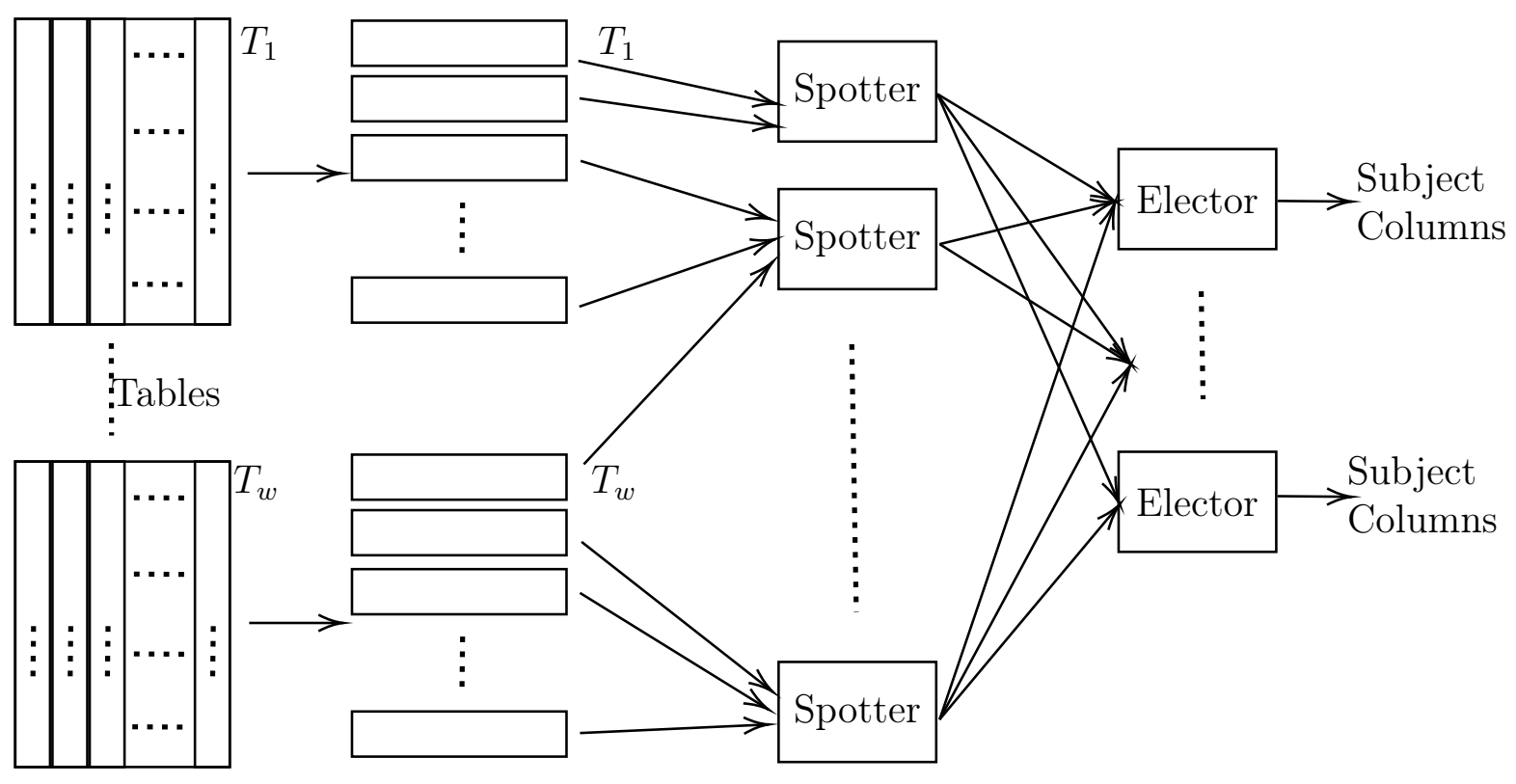

Figure 5-5: Subject Column Detection

\subsubsection{Splitter}

Before splitting the files, each file is assigned an elector instance. This is done in a round-robin manner, so each file is assigned to an elector.

$$
\text { elector }\left(\text { file }_{i}\right)=i \bmod \| \text { electors } \|
$$

The operator mod is the modulus (the remainder of the division), $i$ is the file order (index), which starts from 0 to the number of files -1 . |lelectors $\|$ represents the number of elector instances. Also, we have a mapping between the elector order (from 0 to $\|$ electors $\|-1$ ) and the address of the elector (URL). This is needed later on by the spotters. After assigning an elector address for each file, we split each file into smaller pieces; each of them is composed of a collection of rows. Each piece will inherit the elector address from its file. After that, each piece will be sent to a spotter in a circular fashion (round-robin) with an elector address, the file name, piece id (from 0 to the number of pieces-1), the total number of slices, the spotter technique, and the elector technique. 


\subsubsection{Spotters}

We present three ways to spot subject columns. The first one is to pick the left-most column regardless of the content $[14,80]$. The second one is to pick the left-most column that is not composed of numeric values (not a numeric column) $[14,80]$. The third method picks the column with the most distinct values and considers that as the subject column [64]. It ignores the numeric columns and columns with strings that have less than four characters on average. Ritze et al. [64] use 100 manually defined regular expressions to detect the different data types (e.g., numbers, strings, coordinates, ...), but these regular expressions are not included in their paper, so we apply the method (most distinct) for all non-numeric columns. Throughout this paper, we refer to these spot techniques as left-most, non-numeric, and most distinct, respectively.

After spotting the subject column using the passed spotter technique, the spotter will send to the passed elector address, the id of the selected subject column, the file name, the slice id (piece id), the total number of slices, and the elector technique.

\subsubsection{Electors}

After the spotters detect the subject column for each partition, each sends the candidate subject column to the corresponding elector. The candidate subject column for each partition of any table $T$ will be sent to the same elector (each table is mapped to an elector). The election process will not start until the candidate subject column for all the slices of the given file are received. So, the elector receives subject column candidates for a given table $T$. From this list of candidates, it elects the subject column for table $T$.

We present two techniques for the election process. We refer to the first technique as blind as it does not regard the meaning of the content. It takes the majority votes suggested by the spotters (the most voted subject column), even if the majority of votes suggest that there is no subject column for the given table. In some cases, where spotters cannot find the subject column (e.g., if there are a lot of numeric 
values in some of the partitions), this technique would suggest that the table given to the system does not have a subject column if the majority of the spotters suggest so. The second technique is similar to the first one but is tilted toward suggesting a subject column even if the majority suggests the absence of the subject column. So it takes the majority, but prefer to choose a subject column if the majority of the spotters suggest that the given table does not have a subject column. In such cases, it will select the second most suggested subject column (if any).

The difference between the two election techniques is that if the majority of the candidates did not include a subject column, the former would conclude that the table has no subject column, while the latter will elect the second most frequent subject column (if any). We refer to these techniques as blind and tilt, respectively.

\subsubsection{Subject Column Labeling Algorithm}

Subject column labeling follows a similar paradigm to the subject column detection. It splits the data of a table $T$ into smaller partitions. Each partition is handled separately by Score. The intermediate scores generated (by Score) for the table $T$ go to a corresponding Combine instance. It consumes the results and then sends them to the Label instance, where it produces the final result (potential classes). We show that for a single subject column in Figure 5-8 and the overall workflow for multiple tables in Figure 5-9.

\subsubsection{Data splitting}

Each table is assigned to a Combine instance. We loop through the combine instances in a circular fashion, and each table is assigned to a Combine instance. So table $T_{i}$ is assigned to Combine $_{j}$, such that:

$$
j=i \bmod \| \text { Combine } \|
$$

where $\|$ Combine $\|$ is the number of Combine instances (this is also known as roundrobin). For each table, the id (index) of the subject column is expected. The subject 
column is then split into $\rho$ partitions. Each of the partitions is then sent to a Score instance.
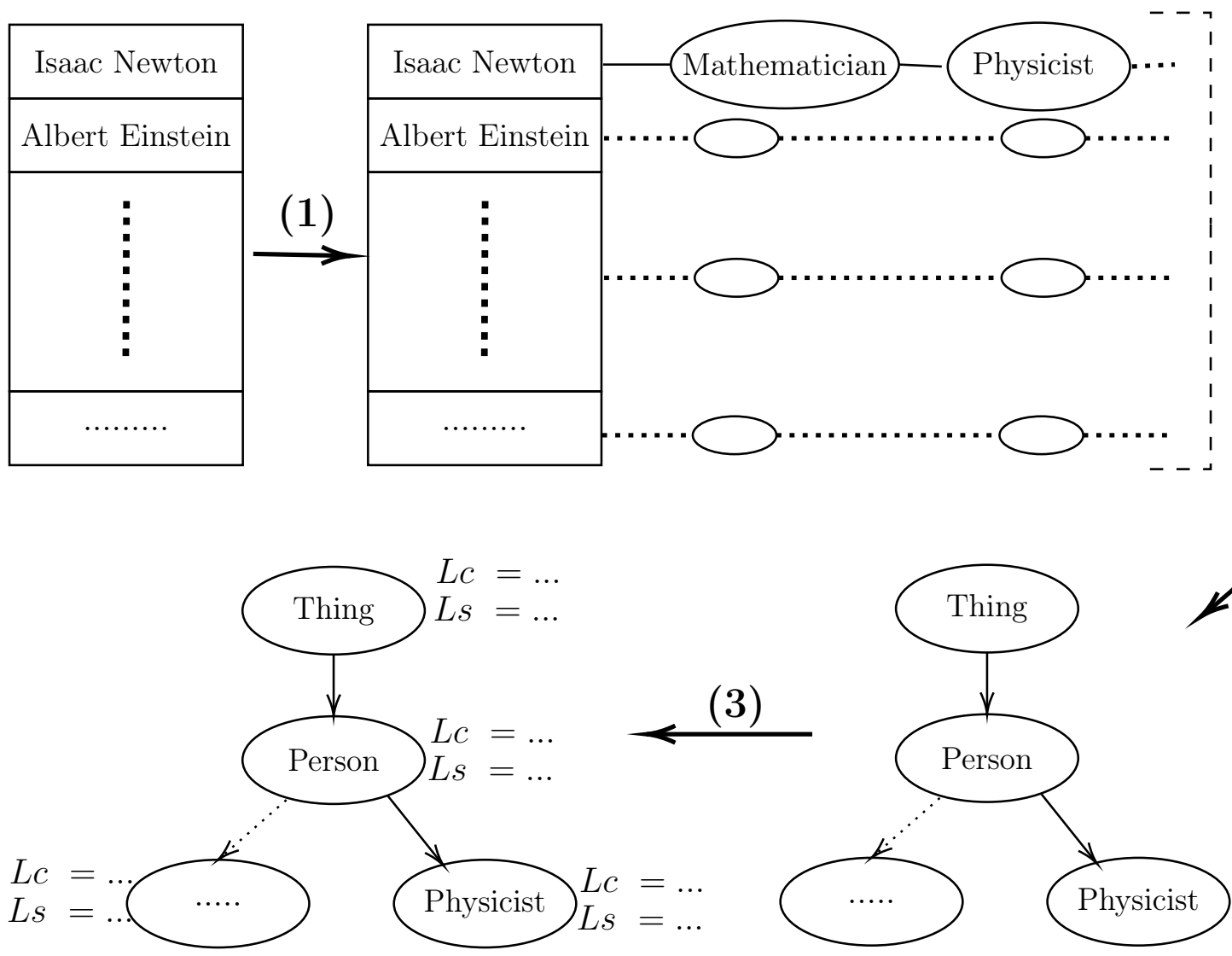

Figure 5-6: Scoring a partition of an entity column

\subsubsection{Partition Scoring}

The input file (that can be very large) is split into smaller parts; we refer to them as partitions. Each partition is scored separately and independently from the other partitions. The scoring functions (and SPARQL queries) used in this step are the same as the ones we use in the initial approach we presented earlier. We show the notation in Table 5.1. This scoring process of a single partition is composed of the following:

1. For each cell, get the potential entities from the knowledge graph (Listing 5.4). 
Listing 5.4: Get potential entities

select distinct ?subject

where $\{$ ?subject ?property "cell_value" \}

Listing 5.5: Get classes of a given entity

prefix dbr: $<$ http://dbpedia.org//resource/ $>$

select distinct ? class

where $\{$ dbr:some-entity a ?class $\}$

2. For each of those entities, get the list of types (Listing 5.5).

3. Build the type hierarchy (using rdfs:subClassOf).

4. For each type (class) in the hierarchy, compute the intermediate coverage (Eq. 5.3) and specificity (Eq. 5.6) scores.

Eq. 5.2 computes how often each type $t$ appears in the column and takes the certainty (the more entities or types assigned to a given cell, the less confidence/score it produces) into account. While Eq. 5.2 is a good estimator, it does not take into account the class hierarchy, so Eq. 5.3 takes it into account. Eq. 5.5 presents how fine or specific a type $t$ is by relying on the ratio of the number of subjects of a type $t$ and its parent class. For example, dbo:Scientist is more specific than dbo: Person. However, Eq. 5.5 only takes into account the direct parent class of the type. To have the specificity on a global scale (on the knowledge graph level) than locally (between the type and its parent), we take into account the full path from the type $t$ to the root (owl:Thing in DBpedia). We multiply the ratio through the whole path Eq. 5.6. The details of these equations are discussed previously in the initial approach (Section 5.1).

\subsubsection{Scores Combining}

The Score instance generates intermediate scores. These scores are associated with each type $t$. We refer to these scores with the generated class hierarchy as "scored class hierarchy". For each partition that goes into the Score instance, it generates a 
scored class hierarchy. The collection of scored class hierarchies for a table $T$ goes to a single Combine instance.

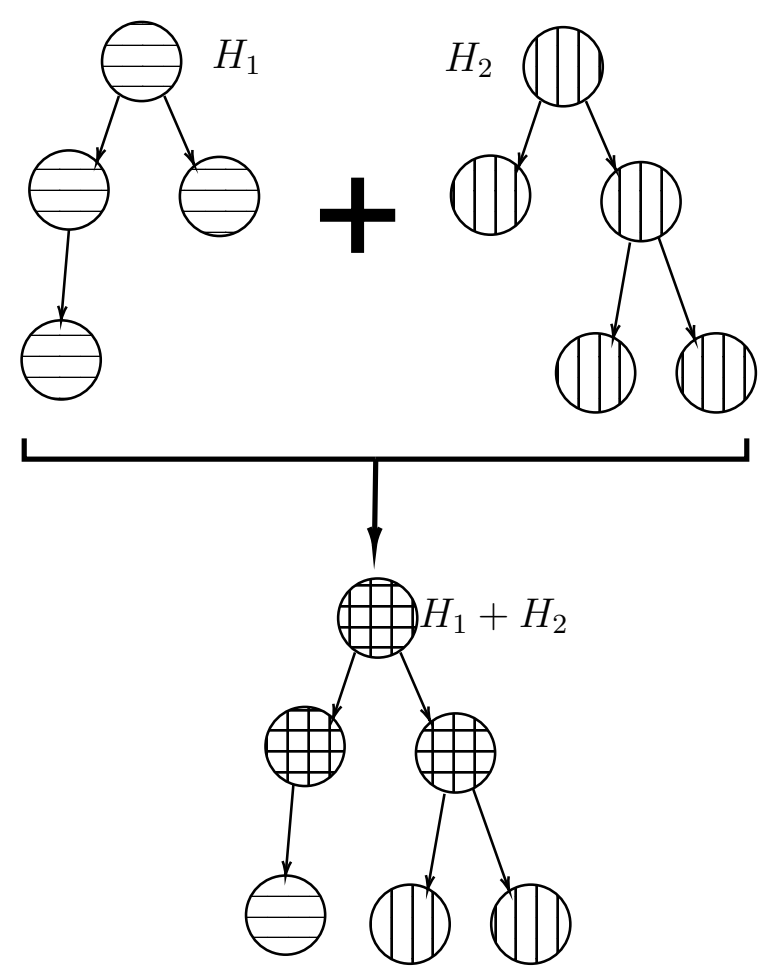

Figure 5-7: Combine the class hierarchy of two partitions

Combine merges these scored class hierarchies into a single scored class hierarchy (Figure 5-7). The way to merge two scored class hierarchies $\left(H_{1}\right.$ and $\left.H_{2}\right)$ is:

1. If a class $\complement$ exists in only one of the two hierarchies, say $H_{2}$, then $\complement$ is included in $H_{1}$ (with its scores) as a child to the parent of $\complement$ (that should exist in $H_{1}$ ).

2. If a class $\complement$ exists in both $H_{1}$ and $H_{2}$, then the $L_{c}(\mathcal{\complement})$ in both is summed, and the $L_{s}(\complement)$ is not changed.

Note that we merge $H_{1}$ and $H_{2}$ into $H_{1}$. The reasoning of both decisions is explained in Section 5.2.5.

\subsubsection{Column Labeling}

This is the last part of the workflow to select the top candidate classes for each subject column. Label takes as an input a scored class hierarchy and then computes the final 


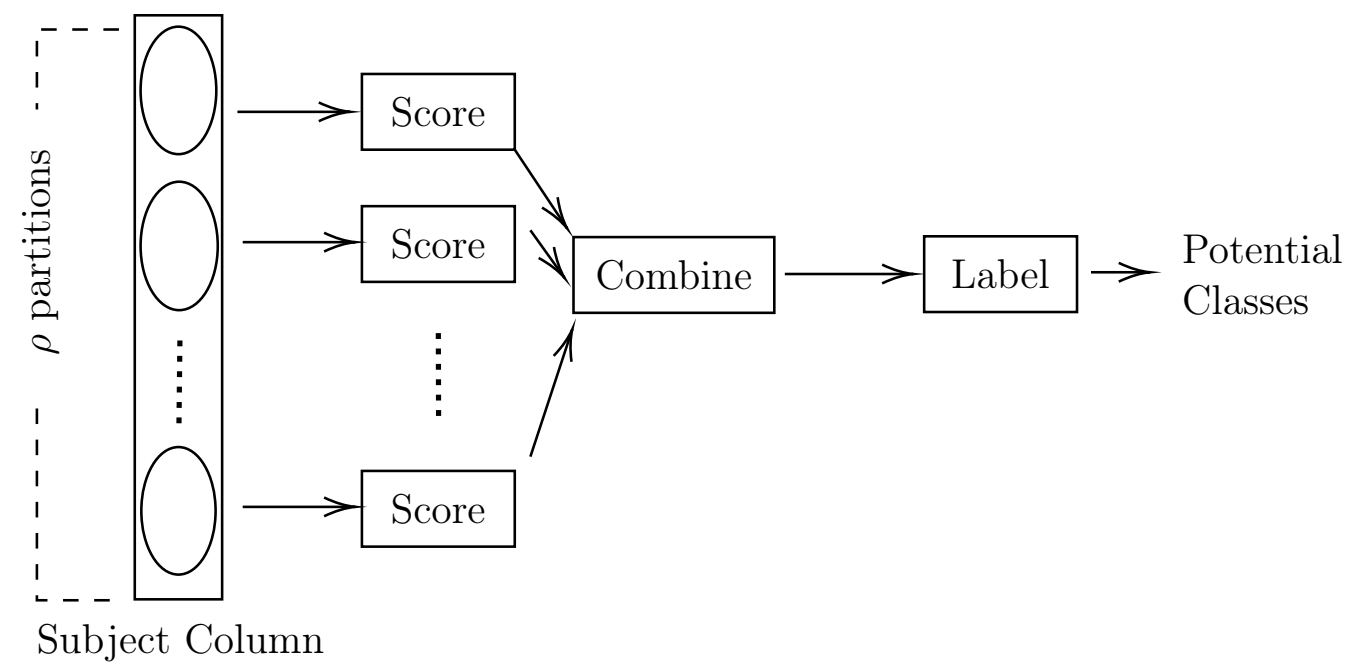

Figure 5-8: Scalable semantic labeling for a single column

score of each type in the hierarchy using Eq. 5.1. It balances two opposite forces, coverage and specificity. In Eq. 5.4, it normalizes the score by dividing the $L_{c}(t)$ by the number of annotated cells $m$. For the specificity function 5.9, it reverts the score of $L_{s}(t)$ to make it yield high when the specificity is high and low when the specificity is low. It also bounds $f_{s}(t)$ between 0 and 1 .

After Label computes the scores for all the types of a given scored class hierarchy, it outputs the highest probable candidates ordered in descending order by the score $f$, Eq. (5.1).

\subsubsection{Experiment Design}

In this section, we show experimentally that using our scalable approach, we achieve the same scores as our initial approach, without any loss in the performance scores. We prove that the scalability of our approach does not cause any loss in precision and recall in Section 5.2.5. Note that we do not measure the speed performance in the scalable approach against the initial approach due to the bottleneck being in querying the English DBpedia. The reason is not only the latency but also that the English DBpedia blocked our requests after several queries. Hence, the scalability in this experiment, we only test whether splitting the data for labeling yields the same score as the initial approach in Section 5.1. 
We developed the application decoupled from the experiment data, so others can use it with their data to ease the comparison with other approaches. The source code is publicly available on GitHub ${ }^{8}$. For this experiment, we used version 1 of the software [2]. We used docker ${ }^{9}$ and docker-compose ${ }^{10}$ to run the services. We have the following services: Spot, Elect, Score, Combine, and Label. We can run multiple services of any of these services at the same time. The services communicate with each other via APIs.

\subsubsection{Setup}

We ran the experiment on Macbook Pro with $2.8 \mathrm{GHz}$ Intel Core i7 with 8 GB DDR3 RAM and with macOS Mojave as the operating system.

For the subject column identification, we created 6 instances of Spot and 3 instances of Elect, both with the partition size of 10 (rows). We ran the tests with different combinations of techniques For Spot, we ran with left-most, non-numeric, and most distinct techniques ${ }^{11}$. The left-most technique spots the left most column regardless of the data, the second technique detects the left-most column that is nonnumeric, and the thirds select the column with the most distinct values. For Elect, we experiment with two techniques: blind and tilt. The former takes the majority decision while the latter picks the majority favoring votes of subject columns over cases where the subject column is not found.

For the first semantic labeling experiment, we had 12 instances of Score, 4 of Combine, and 1 for Label. We have the number of Combine instances less than the number of Score instances as the Combine instances do not need to query the endpoint and hence is faster (to process the partitions). For the Label, we only have 1 instance as it only applies the equations: Eq. 5.4, Eq. 5.9 and Eq. 5.1 and then picks the top candidates (classes with highest scores). Each subject column is split

\footnotetext{
${ }^{8}$ https://github.com/oeg-upm/tada-gam

${ }^{9}$ https://www. docker.com/

${ }^{10}$ https://docs.docker.com/compose/

${ }^{11}$ Note that there are other subject column spotting techniques in the literature. We selected these spotters because they do not need to query the knowledge graph, which is also beneficial for the scalability of the approach.
} 
into multiple partitions of size 10 (or less if the number of elements in a subject column is not divisible by 10). The partition size does not affect the results, as shown in Section 5.2.5. In the second semantic labeling experiment, we use 6 instances of Score, 1 for Combine, and 1 for Label. We use a subset of the values in each subject column instead of using all the values. We used only the first partition for each subject column instead of using all the partitions. This is the reason why we chose only 6 instances of Score. For the Combine, we only used 1 instance, as there are no combination, it only passes the graphs received from the Score instances as is to the Label instance. In the semantic labeling experiments, the correct subject column is picked from the corresponding gold standard. The labeling for a subject column is considered correct if it matches the corresponding class URI in the gold standard; otherwise, it will be considered wrong.

\subsubsection{Experiment Results and Discussion}

We report the results of detecting subject columns with different combinations of spot and election methods. Next, we report two cases of semantic labeling of subject columns: using the whole data and using a single partition (subset) of each subject column.

\subsubsection{Subject Column Detection}

We report the results of subject column detection on T2Dv2 in Table 5.4. The performance of subject columns of T2Dv2 was not reported [63].Thus, we only report the performance of the different techniques for T2Dv2. We see that taking the first non-numeric column from the left out-performs taking the left-most column blindly for both election techniques. Picking the column with the most distinct values from the non-numeric columns yields much higher scores ( $9 \%$ increase in precision and $5 \%$ increase in F1 score compared to the non-numeric spotters with the electors having a minimal effect of $1 \%$ for the non-numeric case). The F1 scores for most distinct and tilt are higher than the combination of (most distinct, blind), with the former being 
Table 5.4: Subject column detection results on T2Dv2 dataset

\begin{tabular}{ccccc}
\hline Spotter & Elector & Precision & Recall & F1 \\
\hline left most & blind & 0.54 & 1.0 & 0.7 \\
left most & tilt & 0.54 & 1.0 & 0.7 \\
non-numeric & blind & 0.86 & 0.98 & 0.91 \\
non-numeric & tilt & 0.86 & 1.0 & 0.92 \\
most distinct & blind & 0.95 & 0.98 & 0.97 \\
most distinct & tilt & 0.95 & 1.0 & $\mathbf{0 . 9 7}$ \\
\hline
\end{tabular}

$0.9 \%$ higher in F1. The combination of most distinct and tilt out-perform all variant techniques presented by [26] when compared using the T2DT dataset. The recall and F1 scores are not reported by [26]. However, to have an idea of the performance and compare their approach (TAIPAN), we computed the F1 scores for the techniques substituting the recall with 1.0 (as if the recall were perfect) and we see that all the techniques in TAIPAN were out-performed in the F1 score as well.

As for the run time, our application took between 14 minutes for the "left-most" spotter technique (for both elector techniques) and around 15 minutes for the "nonnumeric" spotter technique for both elector techniques. For the "most distinct" for both electors, the run time was close to 16 minutes. The elector technique does not seem to affect run time, while the spotter techniques have a small effect. This is reasonable given that "left-most" is $\mathrm{O}(1)$ while "non-numeric" and "most distinct" are $\mathrm{O}\left(\mathrm{n}^{*} \mathrm{~m}\right)$ ( $\mathrm{n}$ as the number of elements in a column and $\mathrm{m}$ is the number of columns). For the elector, it is $\mathrm{O}(\mathrm{m})$ for both techniques.

Despite the fact that this approach seems to work well in practice, it does not take into account the content beyond the simple type checking. For cases where higher accuracy is required, and the performance does not matter, other approaches might be more suitable, like the one presented by Venetis et al. [80]. 
Table 5.5: Subject column detection with T2DT

\begin{tabular}{cccc}
\hline Approach & Precision & Recall & F1 \\
\hline left most + blind & 0.58 & 1.00 & 0.73 \\
left most + tilt & 0.58 & 1.00 & 0.73 \\
non-numeric + blind & 0.86 & 0.97 & 0.91 \\
non-numeric + tilt & 0.85 & 1.00 & 0.92 \\
most distinct + blind & 0.94 & 0.97 & 0.95 \\
most distinct + tilt & 0.94 & 1.00 & $\mathbf{0 . 9 7}$ \\
\hline TAIPAN (Rule-based) & 0.51 & - & $0.68^{*}$ \\
TAIPAN (Support) & 0.54 & - & $0.7^{*}$ \\
TAIPAN (Connectivity) & 0.36 & - & $0.53^{*}$ \\
TAIPAN (Support+Connectivity) & 0.56 & - & $0.72^{*}$ \\
TAIPAN (SVM) & 0.81 & - & $0.90^{*}$ \\
TAIPAN (KNeighbors) & 0.37 & - & $0.54^{*}$ \\
TAIPAN (SGD) & 0.34 & - & $0.5^{*}$ \\
TAIPAN (Decision Tree) & 0.73 & - & $0.84^{*}$ \\
TAIPAN (Gradient Boosting) & 0.76 & - & $0.86^{*}$ \\
TAIPAN (Nearest Centroid) & 0.51 & - & $0.68^{*}$ \\
\hline
\end{tabular}

${ }^{*} \mathrm{~F} 1$ measure is computed with the assumption that recall $=1.0$

\subsubsection{Semantic Labeling}

In Section 5.1.3, we presented that our scoring functions are able to find the correct type. We proved that the results of our distributed semantic labeling would yield the same results as the one presented in that section. We also tested it experimentally and got the same results (Table 5.6). We report the results of our approach in Table 5.6 and denote it as "Our approach (All)".

However, we notice that due to the high frequency of queries to the live DBpedia, the endpoint blocked our queries numerous times during the experiment. As a countermeasure, we introduced a randomized waiting time for requests that are blocked by the endpoint. This affected the run time of the experiment (total run time was 28 hours approximately). 
Table 5.6: Semantic labeling scores of T2Dv2

\begin{tabular}{lccc}
\hline Approach & Precision & Recall & F1 \\
\hline T2K (Majority) & 0.47 & 0.51 & 0.49 \\
T2K (Majority + Frequency) & 0.87 & 0.90 & 0.89 \\
T2K (Page attributes) & 0.97 & 0.37 & 0.53 \\
T2K (Text) & 0.75 & 0.34 & 0.46 \\
T2K (Majority + Frequency + & 0.90 & 0.86 & 0.88 \\
Page attributes + Text) & 0.93 & 0.91 & $\mathbf{0 . 9 2}$ \\
T2K Extended & 0.84 & 0.93 & 0.88 \\
Our approach (Partition) & 0.91 & 0.97 & $\mathbf{0 . 9 4}$ \\
Our approach (All) & & & \\
\hline
\end{tabular}

We compare our results with the reported scores of Ritze et al. [63] on the same data (T2Dv2). We can see that although one of the T2K approaches yielded higher precision at 0.97, our approach outperforms all the approaches reported in [63] in both recall and F1 scores. We also found a few typos in the gold standard that we had to fix ${ }^{12}$.

We also reported the results for testing a subset for each subject column instead of including all the contents of the subject column. In the previous test, we split each subject column into $\rho$ partitions, a single partition for each subject column. In this sampling, we took only the first partition and labeled that partition. We got a very good scoring considering that only a single partition was used. The F1 score obtained was 0.88 , so only two out of the six approaches of T2K out-performed it. We denote it in Table 5.6 as "Our approach (Partition)."

In the end, our approach yielded high scores adding semantic labels from a given knowledge graph in an automatic way without relying on any external linguistic resources and thus is likely to generalize better across datasets and knowledge graphs.

\footnotetext{
${ }^{12}$ The gold standard with the fixed typos can be found in the same repository as the source code of our application in GitHub https://github.com/oeg-upm/tada-gam
} 


\subsubsection{Scoring Equivalence to the Initial Approach}

In this section, we prove that merging the scores column slices yields the same as the scores for the column as a whole. We proof that for the coverage and specificity scoring functions. We explain the used symbols in Table 5.1.

\subsubsection{Coverage}

Lemma 10. Given a list of cells (values) of a column that are split into $\rho$ partitions, computing the $I_{c}$ for each partition separately and average them, yields the same $I_{c}$ for the combined partitions.

$$
I_{c}(t)=\sum_{\varrho}^{\rho} I_{c \varrho}(t)
$$

Proof. Let us say that we have a column $a$ that is split into $\rho$ partitions.

$$
\begin{gathered}
\Psi_{a}=\Psi_{a_{1}} \cup \Psi_{a_{2}} \cup \ldots \cup \Psi_{a_{\rho}} \\
I_{c_{a}}(t)=\sum_{v}^{\Psi_{a}} \sum_{e} \frac{1}{\|Z(v)\| *\|Q(e)\|} \\
I_{c_{a}}(t)=\sum_{v}^{\Psi_{a_{1}} \cup \ldots \cup \Psi_{a_{\rho}}} \sum_{e} \frac{1}{\|Z(v)\| *\|Q(e)\|} \\
I_{c_{a}}(t)=\sum_{v}^{\Psi_{a_{1}}} \sum_{e} \frac{1}{\|Z(v)\| *\|Q(e)\|}+ \\
\sum_{v}^{\Psi_{a_{2}}} \sum_{e} \frac{1}{\|Z(v)\| *\|Q(e)\|}+\ldots \\
\ldots+\sum_{v}^{\Psi_{a_{e}}} \sum_{e} \frac{1}{\|Z(v)\| *\|Q(e)\|}
\end{gathered}
$$




$$
I_{c_{a}}(t)=\sum_{\varrho}^{\rho} I_{c a \varrho}(t)
$$

Lemma 11. Given a list of cells (values) of a column that are split into $\rho$ partitions, computing the local coverage $L_{c}$ for each partition separately and average them, yields the same $L_{c}$ for the combined partitions.

$$
\sum_{\varrho}^{\rho} L_{c \varrho}(t)=L_{c}(t)
$$

Proof. Let us say that we have a column $a$ that is split into $\rho$ partitions.

$$
\begin{gathered}
\Psi_{a}=\Psi_{a_{1}} \cup \Psi_{a_{2}} \cup \ldots \cup \Psi_{a_{\rho}} \\
L_{c}(t)=I_{c}(t)+\sum_{u \in U(t)} L_{c}(u) \\
L_{c_{\varrho}}(t)=I_{c \varrho}(t)+\sum_{u \in U(t)} L_{c \varrho}(u) \\
\sum_{\varrho}^{\rho} L_{c_{\varrho}}(t)=\sum_{\varrho}^{\rho}\left[I_{c \varrho}(t)+\sum_{u \in U(t)} L_{c \varrho}(u)\right] \\
\sum_{\varrho}^{\rho} L_{c_{\varrho}}(t)=\sum_{\varrho}^{\rho} I_{c \varrho}(t)+\sum_{\varrho}^{\rho} \sum_{u \in U(t)} L_{c \varrho}(u) \\
\sum_{\varrho}^{\rho} L_{c_{\varrho}}(t)=\sum_{\varrho}^{\rho} I_{c \varrho}(t)+\sum_{\varrho}^{\rho} L_{c \varrho}\left(u_{1}\right)+L_{c \varrho}\left(u_{2}\right)+\ldots \\
\text { where } U(t)=u_{1}, u_{2}, \ldots
\end{gathered}
$$




$$
\begin{gathered}
\text { and since } L_{c_{\varrho}}(t)=I_{c \varrho}(t)+\sum_{u \in U(t)} L_{c \varrho}(u) \\
\sum_{\varrho}^{\rho} L_{c_{\varrho}}(t)=\sum_{\varrho}^{\rho} I_{c \varrho}(t)+\sum_{\varrho}^{\rho}\left(I_{c \varrho}\left(u_{1}\right)+\ldots+I_{c \varrho}\left(u_{2}\right)+\ldots\right) \\
\sum_{\varrho}^{\rho} L_{c_{\varrho}}(t)=\sum_{\varrho}^{\rho} I_{c \varrho}(t)+\sum_{\varrho}^{\rho} I_{c \varrho}\left(u_{1}\right)+\sum_{\varrho}^{\rho} I_{c \varrho}\left(u_{2}\right)+\sum_{\varrho}^{\rho} \ldots \\
\text { and since } I_{c_{a}}(t)=\sum_{\varrho}^{\rho} I_{c a \varrho}(t)(\operatorname{lemma} 10) \\
\sum_{\varrho}^{\rho} L_{c_{\varrho}}(t)=I_{c}(t)+I_{c}\left(u_{1}\right)+I_{c}\left(u_{2}\right)+\ldots \\
\sum_{\varrho}^{\rho} L_{c_{\varrho}}(t)=I_{c}(t)+\sum_{u \in U(t)} L_{c}(u) \\
\sum_{\varrho}^{\rho} L_{c_{\varrho}}(t)=L_{c}(t)
\end{gathered}
$$

\subsubsection{Specificity}

For the specificity, the $L_{c}(t)$ is only dependent on the knowledge graph. Let us say we have $L_{c}(t)$ for two partitions, we refer to them as $L_{c 1}(t)$ and $L_{c 2}(t)$ for a type $t$. Each depend on the $L_{c}(\operatorname{pr}(t))$ of the parents and the $I_{s}(t)$. Since the knowledge graph is the same in both, the number of instances for any type is the same. Hence, $L_{s 1}(t)$ and $L_{s 2}(t)$ have the same value. So when merging two scored hierarchies, the values 
of $L_{s}$ is not summed, instead, only one of them is picked. 


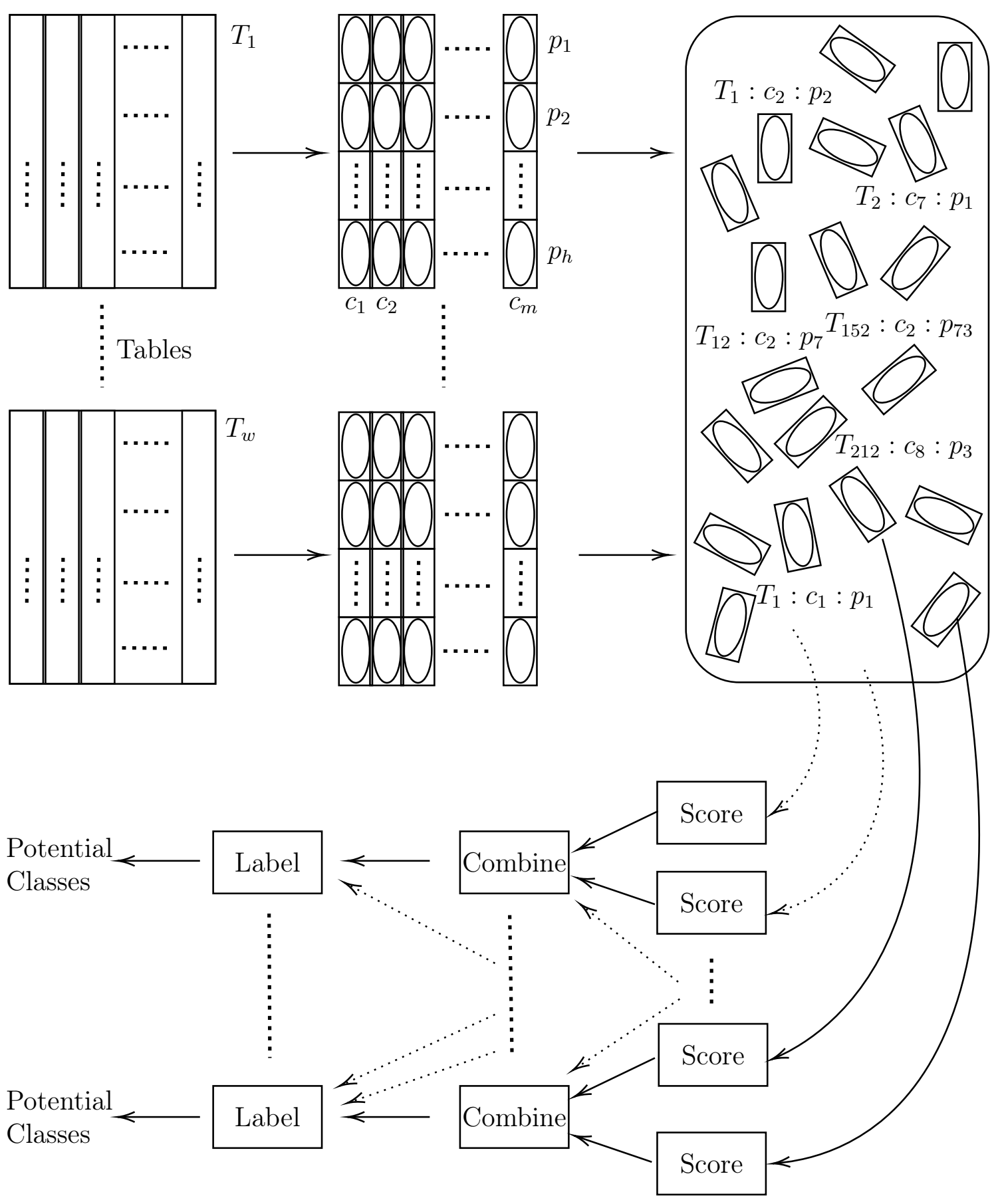

Figure 5-9: Scalable semantic labeling for multiple tables 


\section{Chapter 6}

\section{Semantic Labeling of Numeric}

\section{Columns}

Semantic labeling (of tabular data) is the process of assigning classes and/or properties from one or more ontologies to columns in tabular data [3,9]. In this chapter, we focus on semantic labeling of numeric columns. This is also referred to as semantic annotation $[36,76,3,9]$. Assigning semantic labels to numeric columns is challenging, because of the large differences that are usually visible between the values of numeric columns and the values of numeric properties in the knowledge graphs. Such differences are sometimes due to measurement accuracy: some measurements have high accuracy (e.g., to five fractions) while others have low accuracy (e.g., two digits fractions). They may also be due to the technology used for the measurement or the approximations applied by the data providers. Another potential reason is the expiry of some data (e.g., the current population of Spain). Some data are updated periodically (population, number of goals of a team), while other data are rarely updated (height of a building).

Some naive approaches tried to solve these discrepancies by adding a threshold (e.g., 0.1). If the difference between any two numbers is small (less than the threshold), they are treated as if they were equal. Others use the distribution of the numeric values to annotate numeric columns. The distribution of a column is compared with the distribution of each numeric property in the knowledge graph. Often, only nu- 
meric properties referring to the same class as the numeric column are checked.

We define the problem statement as follows: given a collection of numerical values, a class describing the content of a table and a target knowledge graph, return the list of properties in the knowledge graph that most likely correspond to those numerical values, ordered by likelihood score.

In this chapter, we present two approaches for the semantic labeling of numeric columns in tabular data: the uniform approach and the typology-based approach. The former treats all numeric collections in the same way while the later treats different types of numbers differently.

\subsection{A Uniform Approach for Semantic Labeling of Numeric Columns}

This approach aims to assign numeric properties from a given knowledge graph to the numeric columns of a given collection of tabular data. The algorithm expects the tabular dataset, a URL of the SPARQL endpoint where the knowledge graph is accessible, and the semantic class of the subject column to annotate the numeric columns of a single table.

\subsubsection{Algorithm Description}

This approach is composed of multiple steps: extract numeric properties from the knowledge graph, build the model, identify numeric columns in the tabular dataset, and assign the numeric properties to the numeric columns. The pseudo code of the algorithm is shown in Algorithm 1.

\subsubsection{Extraction of Numeric Properties from a Knowledge Graph}

For this step, we explored three different ways to extract numeric properties. The first method relies on the T-box (ontology), while the other two use the A-box (data).

Extraction method 1: in this method, we use rdfs:range to extract numeric 


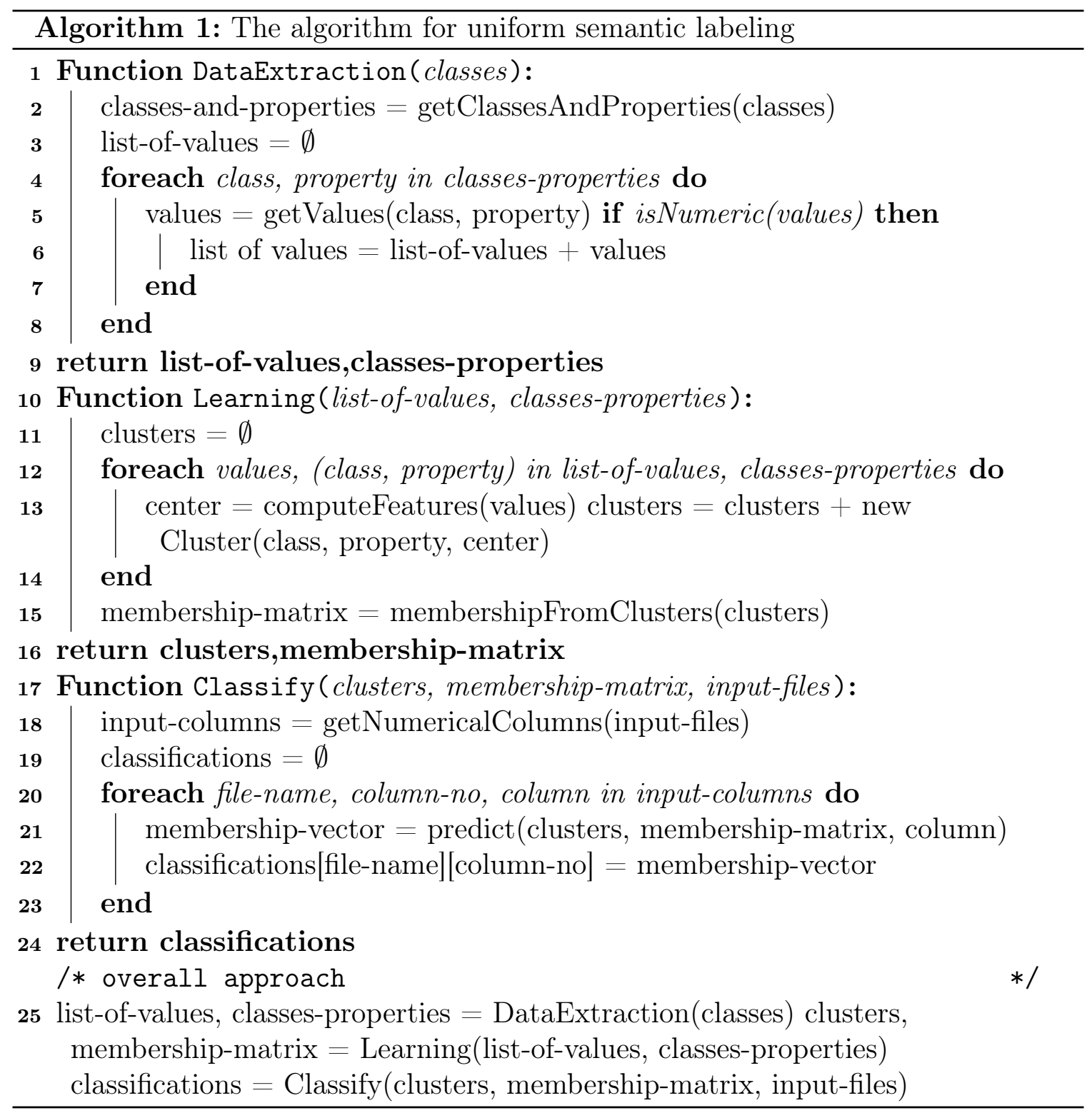




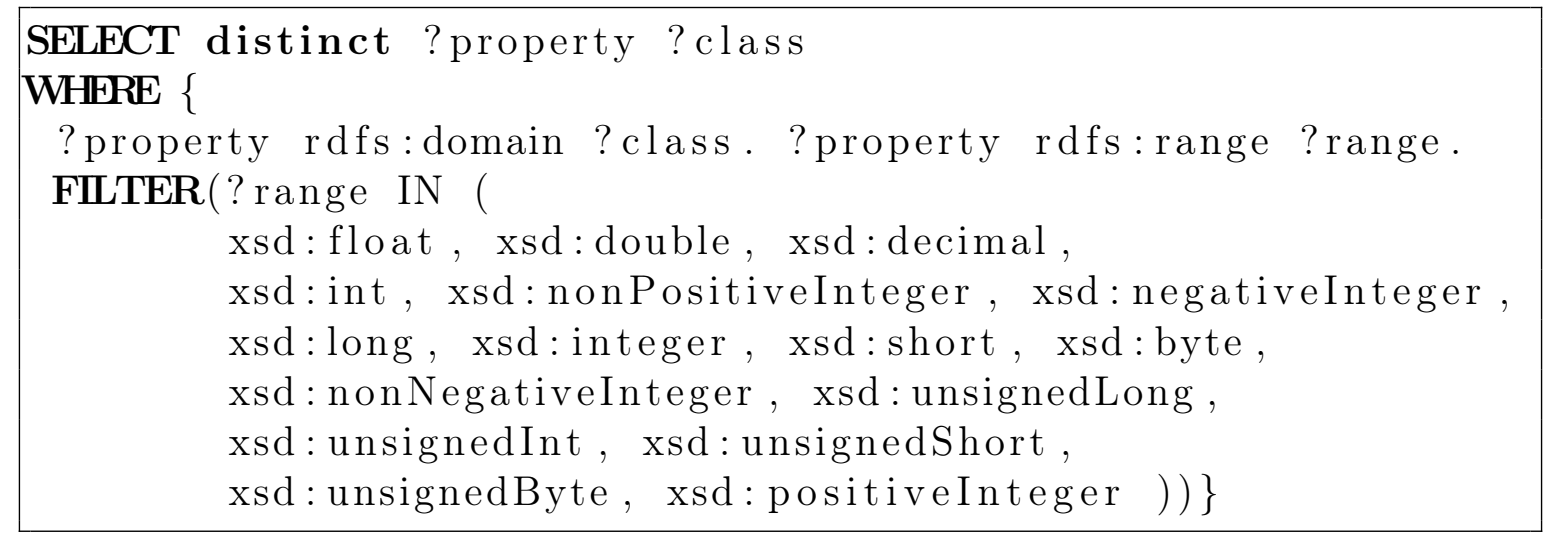

Listing 6.1: Query to extract numeric properties (extraction method 1)

SELECT ? property, COUNT(DISTINCT ? object) AS ? cnt

WHERE \{ ? subject ? property ?object.

FILTER ( isNumeric (? object ) )

\} GROUP BY ? property

Listing 6.2: Query to extract numeric properties (extraction method 2) for all classes, together with the number of distinct values

properties from the knowledge graph (Listing 6.1). Although this method seems to be a good way to extract such numeric properties, it did not work well in practice. We tested this on the English DBpedia, and a lot of numeric properties were missing, since a lot of properties do not have their range ( $r d f s$ :range) defined.

Extraction method 2: this method relies on the data in the knowledge graph. The used query checks for properties that have numeric values (Listing 6.2). However, there is an important limitation in this case, the evaluation of this query times out for a knowledge graph like DBpedia. This is also reported by Neumaier et al. [52]. We also tried it for every single class (Listing 6.3), but it usually timed out for many classes as well (we substituted "classuri" with the actual class URI).

Extraction method 3: this method also uses the A-box. The query (Listing 6.4) asks for all the properties of a single class ("classuri"). Then, for each property, we check if it is numeric or not. We do that by first getting the values for each property propertyuri (Listing 6.5). After that, we check if more than $50 \%$ of the values (objects) are numeric. If so, then we consider the property numeric; otherwise, the property will be considered non-numeric and will be ignored. This extraction 
SELECT ? property WHERE \{

?subject a $<$ classuri $>$.

?subject? property ?val

FILTER( is Numeric (? val))

\} GROUP BY ? property

Listing 6.3: Query to extract numeric properties (extraction method 2) for a single class

\section{SELECT distinct ? property WHERE \{ \\ ?subject a $<$ classuri $>$. \\ ?subject ?property []. \\ \} GROUP BY ? property}

Listing 6.4: Query to extract properties (extraction method 3) for a single class

method is superior to the previous two approaches for two reasons. The first one is that it is faster and does not timeout. The second reason is that it is more accurate (compared to the 2nd extraction method). That is because it checks if more than half of the values are numeric, while the 2 nd method only checks if the property has a single numeric value, which is often not accurate as it does not take in to account the usual noise in the knowledge graph.

\subsubsection{Model Construction}

Once the numeric properties and their values are extracted from the knowledge graph, we build a model for the given class. The model is created from the features for all the numeric properties (of the given class). We use two features for each property of the class: the mean and the standard deviation (std). The features are computed for each property using the corresponding numeric values. Each property corresponds to a cluster, and the features of each property correspond to the centers for that cluster

\section{SELECT ? object WHERE \{ \\ ? subject a $<$ classuri $>$. \\ ?subject <propertyuri $>$ ? object. $\}$}

Listing 6.5: Query to extract objects for a class and property combination (extraction method 3) 
i.e., each feature is a dimension (Figure 6-1). As shown in Figure 6-1, the x-axis represents the mean, and the y-axis represents the standard deviation.

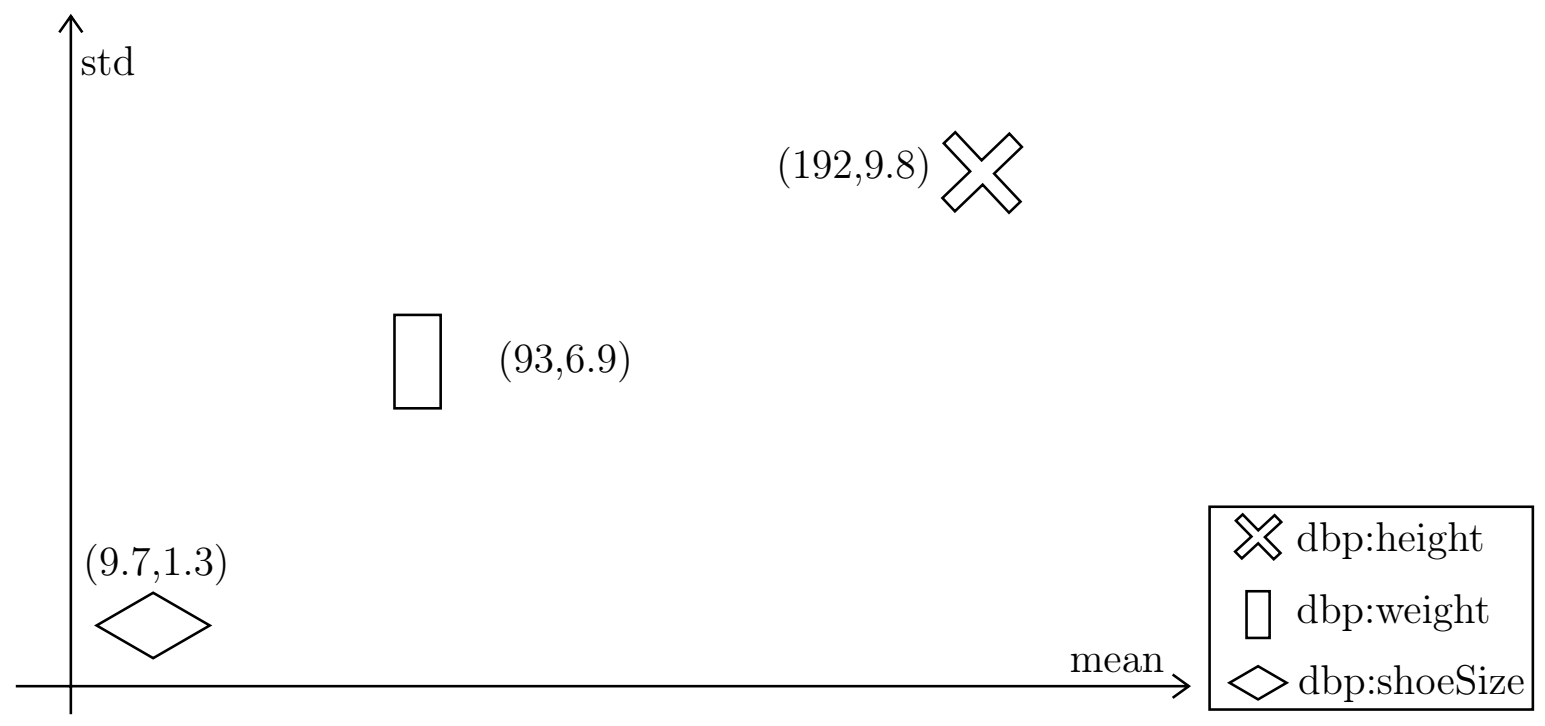

Figure 6-1: Example of cluster centers of a model of the class dbo:footballPlayer

\subsubsection{Property Matching}

When a table is fed to the system to be labeled, the algorithm starts by detecting its numeric columns. This is done by examining the values of each column. A column is considered numeric if the majority of its values are numbers. Non-numeric columns are ignored.

Features (mean and standard deviation) are computed for all the detected numeric columns. Then, the model is used to compute the membership vector for each column using Eq. 2.2.

With each numeric column having their computed features, we compute the membership vector using the computed features (Eq. 2.2). The result is a membership vector for each numeric column. A membership vector includes the membership value for each corresponding cluster center (numeric property). Membership values are between 0 and 1 i.e., the sum of the membership values for any membership vector should be 1 . The membership value represents the belonging of the numeric column 
Table 6.1: Membership Vector Example

\begin{tabular}{cc}
\hline Cluster & Membership \\
\hline$d b p:$ weight & 0.8 \\
$d b p:$ height & 0.15 \\
$d b p:$ shoeSize & 0.5 \\
\hline
\end{tabular}

to the corresponding cluster center (numeric property). Higher values of membership indicate higher belonging to the corresponding clusters.

For example, if we have three clusters, dbp:height, dbp:weight, and dbp:waist, a membership vector of a numeric column can be something like this $<0.15,0.80,0.05>$. This means that the given column belongs $15 \%$ to the $d b p$ :height cluster, $80 \%$ to the $d b p$ :weight cluster, and $5 \%$ to the dbp:shoeSize cluster (Table 6.1).

Note that this differs from the classical fuzzy c-means. In the classical version, membership matrix is set randomly. Then, the center for each cluster is computed as they are not known before hand. The membership matrix is then updated according to the new centers of the clusters. The computing of centers and membership matrix is repeated until the change in the membership tiny (less than a given threshold). In our case, the cluster centers are set at the beginning as we know the clusters (each cluster corresponds to a property). This is done using the features computed for the values of each property. Then, the membership vector for each column is computed. This is performed using the features computing using the values in the column. Each of these steps (computing the centers of the clusters and computing the membership matrix) is done only once, in contrast to the classical version.

For each numeric column, the membership vector is ordered in descending order, and the top corresponding cluster centers (numeric properties) are picked for the property assignment. 


\subsubsection{Experiment Design}

In this section, we aim to answer the research question Q.2: whether we can assign semantic labels to numeric columns in the absence of context using a given knowledge graph. By context here, we refer to meta data, data which are not included under any of the columns (e.g., URL, caption).

To do so, we implemented our algorithm to assign semantic labels to the numeric columns using a knowledge graph. We implemented our own fuzzy c-means library ${ }^{1}$, following the work of Bezdek et al. [10]. The implementation takes as an input the table and the corresponding class URI. The class URI represents the class of the subject column in terms of the ontology used in the SPARQL endpoint. The application takes the class URI, extracts the relevant numeric properties, builds the model, detects the numeric columns, and then assigns a list of potential properties to each. For each numeric column, the most relevant $\mathrm{k}$ labels are returned; that is the ones with the highest membership values. We considered the job of assigning labels as correct if the label from the gold standard matches one of the top-k suggested labels. We report precision at different values of $\mathrm{k}$ (precision@k).

We used two (manually annotated) datasets for our experiment, Olympic Games [4] and T2Dv2 ${ }^{2}$.

For each dataset, we measured the performance of our algorithm using the precision@k score. The precision is computed by dividing the number of correctly labeled columns over the total number of labeled columns (Eq. 6.1). For the recall@k, it is computed by dividing the number of correctly labeled columns over the total number columns that should have been labeled (Eq. 6.2). However, the algorithm will annotate all the numeric columns in the gold standard, and the recall will always be 1.We also report the F1@k score (Eq. 6.3). The source code of the application and the used datasets are published [6].

$$
\text { precision }=\frac{\# \text { correct }}{\# \text { correct }+\# \text { incorrect }}
$$

\footnotetext{
${ }^{1}$ https://github.com/oeg-upm/fuzzy-c-means

${ }^{2}$ http: //webdatacommons . org/webtables/goldstandardV2.html
} 


\begin{tabular}{ccccc}
\hline $\mathrm{k}$ & Approach & Precision@k & Recall@k & F1@k \\
\hline \multirow{2}{*}{1} & FCM & 0.96 & 1.0 & 0.98 \\
& Random & 0.0025 & 1.0 & 0.005 \\
\hline \multirow{2}{*}{3} & FCM & 0.96 & 1.0 & 0.98 \\
& Random & 0.0075 & 1.0 & 0.015 \\
\hline \multirow{2}{*}{5} & FCM & 0.96 & 1.0 & 0.98 \\
& Random & 0.0125 & 1.0 & 0.025 \\
\hline
\end{tabular}

Table 6.2: Classification scores of the numeric columns of Olympic Games

$$
\begin{aligned}
\text { recall } & =\frac{\# \text { correct }}{\# \text { correct }+\# \text { notfound }} \\
F 1 & =\frac{2 * \text { precision } * \text { recall }}{\text { precision }+ \text { recall }}
\end{aligned}
$$

\subsubsection{Results and Discussion}

We report the scoring results for the Olympic Games dataset in Table 6.2. We refer to our approach as FCM (fuzzy c-means). We can see the precision@1 is high (0.96). Usually, we expect the precision to increase as the value of $k$ increases, but in this test, there was a property that did not show up in the top 5 . To provide an idea about the difficulty of getting the correct numeric property by chance, we compare the results we got with the probability of assigning the correct property from DBpedia to the numeric columns (precision). We also report the recall as 1 , since we are only interested in getting a correct property and not all related properties. We used this precision@k, recall@k, and F1@k of getting the correct label at random as a baseline. We computed that for each numeric column, and then took the mean. It shows that it is very unlikely to assign the numeric columns with the correct properties from DBpedia. For example, the probability of getting the correct property for a given column by change is 0.0025 . 


\begin{tabular}{ccccc}
\hline $\mathrm{k}$ & Approach & Precision@k & Recall@k & F1@k \\
\hline \multirow{2}{*}{1} & FCM & 0.34 & 1.0 & 0.51 \\
& Random & 0.0004 & 1.0 & 0.0008 \\
\hline \multirow{2}{*}{3} & FCM & 0.55 & 1.0 & 0.71 \\
& Random & 0.001 & 1.0 & 0.0020 \\
\hline 5 & FCM & 0.83 & 1.0 & 0.91 \\
& Random & 0.002 & 1.0 & 0.004 \\
\hline \multirow{2}{*}{10} & FCM & 0.91 & 1.0 & 0.95 \\
& Random & 0.004 & 1.0 & 0.0080 \\
\hline
\end{tabular}

Table 6.3: Classification scores of the numeric columns of T2Dv2

We also tested with a bigger dataset, T2Dv2. We report precision scores in Table 6.3. We see that precision is lower compared to the test on the Olympic Games dataset. Also, the probability of assigning the correct properties to the numeric columns is even lower.

The scoring results of the Olympic Games are much higher than the scores of T2Dv2. Looking closely at the data, we can see that numerical data contained in the Olympic Games tend to be close to a normal distribution (e.g., the weight of soccer players). Normal distributions are commonly represented by the mean and variance. Looking at the features we used, we use the mean and the standard deviation, which is the square root of the variance. For example, looking at the population density of countries, data are concentrated more on the left, which looks close to a chi-squared distribution with $\mathrm{k}=2$. Such numerical properties are harder to classify. It also depends on the distance to other clusters. Some clusters are very close to each other, which results in misclassifications. In such a case, the points are matched to a nearby but wrong cluster. An example of such cases is the two clusters areaOfCatchment and elevation of class dbo:Lake. Sometimes this occurs with irrelevant properties (e.g., wikiPageID or imageSize).

Another thing we noticed is how properties that represent years are difficult to 
understand. Given a collection of values that represent years, it is hard to guess whether they represent dates of birth of people or dates of football matches. Even with such properties that are easy to mix up, there may be some influence. For example, the birth dates of people are before their death dates. Nonetheless, machine learning techniques often cannot distinguish between birth dates and death dates [76]. The nature of the data used in the model also influences the classification score. For example, if we have two date-related properties, such as birth date (year) of Nobel prize holders, and birth dates (year) of young Internet millionaires, most probably, the classification would not be mixing the two. The first ones are generally older (less) than the birth dates of the young internet millionaires.

In addition, our approach is domain agnostic. Our approach is not bound to a specific knowledge graph; it queries whatever endpoint passed to it. The model is constructed using the provided SPARQL endpoint, without any change in the program. It only has to include data that are of the same type that exists in the input files to be classified. Since our approach accepts concepts that are related to the input files, there is no need to explore and include other unrelated concepts in the model. So, only related concepts are included in the model (which is used for the classification). Another advantage of our approach is that it does not require the numeric values in the numeric columns and properties (in the knowledge graph) to match. The approach focuses on how the values reside in the space rather than to which entity these values belong. Moreover, the learning process is semi-automatic (or automatic if the classes are provided, like in the case of Web Tables from Web Data Commons). Despite the fact that concept discovery is not automatic (the user has to provide the concepts as an input for the model construction), the tasks for assigning the labels are done automatically, such as the lookup for numeric properties, the model construction, and the assignment of numeric properties. Ergo there is no need to train the model manually by matching columns in input files to properties in endpoints. 


\subsection{Typology-based Approach for Semantic Labeling of Numeric Columns}

The approach presented in Section 6.1 did not differentiate between different types of numeric columns, treating all of them in the same manner.

In this section, we go a step further by considering that values in numeric columns may have different characteristics. We first characterize different types of numeric values. Then, we use this typology to improve the performance of our semantic labeling approach. We show the workflow in Figure 6-2. We refer to the implementation of this approach as TTLA.

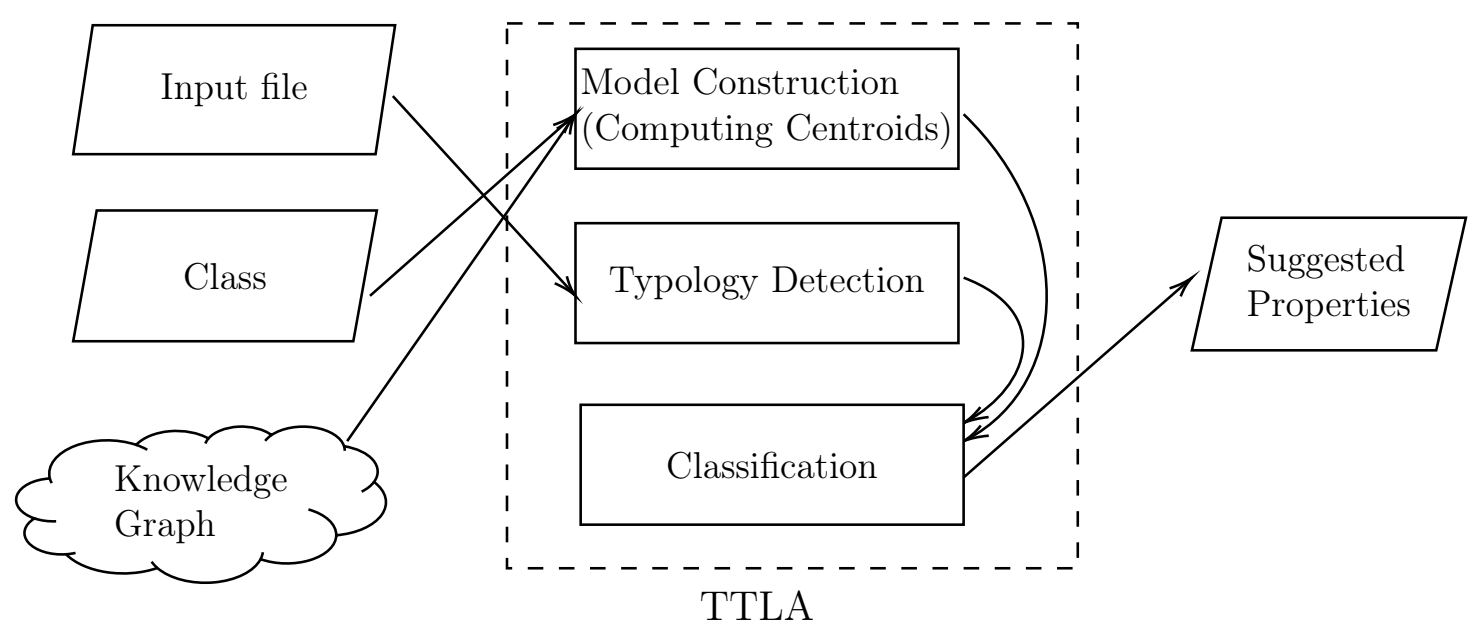

Figure 6-2: The workflow of typology-based semantic labeling

\subsubsection{Typology of Numeric Columns}

We adopt the typology presented by Stevens et al. [72] as a base, and we build on top of it; because it is more suitable for the detection of different types. We extend two of the high-level types (nominal and ratio) into sub-types. We based our work on types discussed by Tukey [78], Mosteller et al. [49], and Stevens et al. [72]. 


\subsubsection{Example}

Figure 6-3 presents a table about military individuals, with several numerical columns, where we assign a type and a sub-type for each of them. The first column contains the first names, so it is not a numeric column. The second column represents the "service name". Each person has a unique service name showing on their uniform. The 3rd column represents the heights in centimeters. After that is the column about the gender, where males are denoted as 1 and females as 2 . The "Race rank" column represents the order of the winners of a race held for those people (e.g., 1 means the person came first, 2 means the person came second place). The sixth column represents the number of goals each of the players scored in a season. The 7th column contains the internal identifiers of the personal files (there is a file about personal information for each person). The following column contains the coldest temperature (in Celsius) of the water in which the corresponding person was able to swim. The last column represents the civil id for each person. We discuss further the construction of the civil ids later in this section (Figure 6-4).

\subsubsection{Nominal}

Nominals are labels that are composed of digits. They are used to distinguish between things represented by different labels. Such are used instead of names because it is easier to check for uniqueness compared to names, as multiple people can share the same name ${ }^{3}$.

It could be impossible to know whether given numerals in a column are nominal or not without having extra context. However, understanding how these numerals are generated can give us some insights.

To ensure the uniqueness, some use a sequence of natural numbers (e.g., military units). Some start from large numbers, and the sequence would be, for example, organized as $(90000,90001,90002, \ldots)$. We refer to such sets of numbers as sequential. In Figure 6-3, an example of a sequential sub-type is presented in the column "Service

\footnotetext{
${ }^{3}$ As the case of baseball https://en.wikipedia.org/wiki/Uniform_number_(Major_League_ Baseball
} 


\begin{tabular}{|c|c|c|c|c|c|c|c|c|}
\hline Name & $\begin{array}{l}\text { Service } \\
\text { number }\end{array}$ & Height & Gender & $\begin{array}{l}\text { Race } \\
\text { rank }\end{array}$ & \begin{tabular}{|} 
Number \\
pf goals
\end{tabular} & File ID & $\begin{array}{l}\text { Coldest } \\
\text { swim }\end{array}$ & Civil ID \\
\hline John & 9008 & 185 & 1 & 1 & 0 & 12034 & 18 & 2900201134 \\
\hline Judy & 9001 & 188 & 2 & 2 & 2 & 34842 & 7 & 3890415293 \\
\hline Nancy & 9005 & 171 & 2 & 3 & 3 & 43833 & 12 & 2881214201 \\
\hline Alex & 9004 & 160 & 2 & 4 & 2 & 83732 & 15 & 7841128284 \\
\hline Jack & 9002 & 210 & 1 & 5 & 43 & 29243 & 6 & 3920820131 \\
\hline Mary & 9003 & 191 & 2 & 6 & 52 & 30152 & 19 & 5940423221 \\
\hline Bob & 9006 & 154 & 1 & 7 & 5 & 18513 & 17 & 1850404118 \\
\hline Mike & 9009 & 187 & 1 & 8 & 18 & 50418 & 10 & 4850327178 \\
\hline Alice & 9007 & 178 & 2 & 9 & 1 & 13312 & 9 & 4911223213 \\
\hline & $\downarrow$ & & $\downarrow$ & & $\downarrow$ & & $\downarrow$ & \\
\hline Sub-types: & {$\left[\begin{array}{c}\text { sequential } \\
,\end{array}\right.$} & $\gamma$ & categorical & 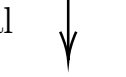 & $\underset{1}{\operatorname{counts}}$ & $r$ & other & 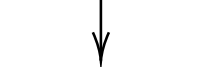 \\
\hline & 1 & other & 1 & ordinal & 1 & random & 1 & hierarchical \\
\hline \multirow{3}{*}{ Types:- } & $\ulcorner\quad 1$ & i & 1 & 1 & \multirow{3}{*}{ interval-rati } & i & \multirow{3}{*}{ interval-rati } & i \\
\hline & \multirow[t]{2}{*}{ [nominal } & I & \multirow{2}{*}{ nominal } & I & & tio & & io \\
\hline & & nterval-ra & & ordinal & & nominal & & nominal \\
\hline
\end{tabular}

Figure 6-3: An example of types and sub-types of numerical columns

number", where the values range from 9001 to 9009 .

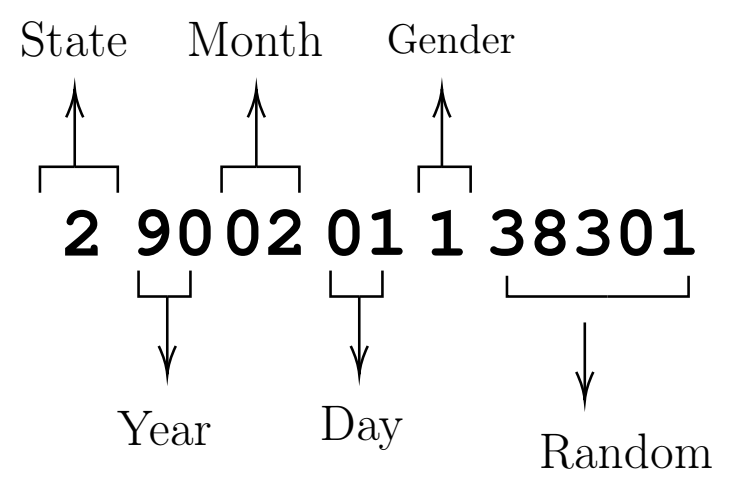

Figure 6-4: An example of hierarchical sub-type

In other cases, the number is a combination of segments, where each segment has a meaning [46]. A segment of a number can mean the unit of the soldier (in the case of military personnel) ${ }^{4}$, the date of birth [46], gender, place of birth, and usually the last part of a such a nominal number ends with a random number or sequence [46].

\footnotetext{
${ }^{4}$ https://en.wikipedia.org/wiki/Service_number
} 
Such are referred to as hierarchical (see Figure 6-4).

The third sub-type of nominal is the general case where a number represents a group, sometimes referred to as categorical. An example of such data can be seen in the column "Gender" in Figure 6-3, where 1 is used to represent a male and 2 to represent a female.

The fourth kind of nominal is something that is either randomly generated (like automatically generated ids in software), or they only make sense for the platform like addresses in memory (column "File ID" in Figure 6-3).

To summarize, we identify four sub-types of nominals: 1) sequential; 2) hierarchical; 3) categorical; and 4) random.

\subsubsection{Ordinal}

It is the same ordinal scale we explained in Section 2.2.2. It represents a rank with no regard to the difference. For example, it is concerned with the order of the winners $(1$ st, 2 nd, ...), but with no regard to how many seconds it took each of the runners to complete the race. It is also not concerned about how much faster the 1st runner from the 2nd (speed difference).

\subsubsection{Interval and Ratio}

We introduce two sub-types: counts and other. Following [72], we define the sub-type counts (also known as cardinal numbers) as the number of instances or occurrences of something. An example of this is the number of goals scored, as shown in Figure 6-3.

In fundamental ratio, the numbers tend to have fewer digits in the fraction parts than derived ratios. This is because they are measured, and the accuracy is bound by measurement tools, while the derived ratio is a function that could produce more fractions (more digits after the decimal point). For example, if we have a circle with a radius of 0.13 (which is a fundamental ratio), and we want to calculate the area of this circle $^{5}$ (derived ratio). Even with $\pi$ rounded to 3.14 , the result is 0.053066 (number

\footnotetext{
${ }^{5}$ area $=0.5 * 2 * \pi * r^{2}$
} 
of digits in the derived ratio is more than the number of digits in the fundamental ratio).

Despite this observation, it is just a matter of limitation that changes over time (as measurement tools get advanced further) and computers advance to handle such numbers. Furthermore, we notice that data published on the web tend to have very few digits after the decimal point. It is common for data owners to round their numbers to a few digits after the decimal point ${ }^{6}$. Hence, we group fundamental ratio and derived ratio in a sub-type we refer to as "other". An example of this sub-type is the human height (Figure 6-3).

For the cyclical ratio, some can fall under the fundamental ratio, while others can fall under the derived ratio (e.g., whether the angle is measured by a protractor or is the output of a function). Therefore, we add the cyclical ratio to the sub-type other.

Moreover, we group ratio and interval together despite the fact that they are different conceptually. However, just looking at a zero, it is impossible to tell whether it is a "real" zero or just an agreement without extra information (which is the difference between ratio and interval). Since we can not tell them apart, we group them together. As the counts are distinguishable from the rest, we will put the interval in the other sub-type. We show an example in Figure 6-3 (column "Coldest swim" represents the coldest temperature they can swim in).

\subsubsection{Algorithm Description}

Our typology-based semantic labeling expects the same input as the uniform approach: the tables (input files) and the classes (of the subject column) for each table. The approach starts by detecting the numeric type for each numeric column. After that, it builds a model and then assigns potential semantic properties from the knowledge graph (Figure 6-2).

\footnotetext{
${ }^{6}$ This observation is data source dependent and does not hold on all datasets.
} 


\subsubsection{Typology Detection}

In this section, we present our approach to detect the typology of numeric columns. The aim is to assign the (sub-) type of any given numeric column. We propose different detection algorithms to detect the different (sub-)types. Our detection algorithms do not assume any knowledge about the numeric values in any column.

\subsection{Nominal}

In general, all nominal numbers are expected to be natural numbers. Let $X$ be the input collection of numbers,

$$
\forall x_{i} \in X ; x_{i} \in \mathbb{N}
$$

Sequential nominal numbers are easier to detect if the data is complete. For example, if we have a list of numbers $X$, with 700 as the minimum value, and 923 as the maximum value, we check if this list is of the sub-type sequential by checking if the list $X$ equals the natural sequence $[700,923]^{7}(Y)$. It becomes tricky when we have missing values due to the selected population having something in common (e.g., sequences of military personnel for a sub-unit). The intuition that we follow is that if more than the square root of the numbers in the sequence $Y$ are also in the original collection of numbers $X$, then we consider the collection of numbers as a sequential collection. So we consider a collection of numbers $\mathrm{X}$ sequential if the following formula is satisfied:

$$
\|X \cap Y\|>\sqrt{\|Y\|}
$$

$\|Y\|$ represents the number of elements in $Y$ and $\|X \cap Y\|$ represents the number of elements that exist in both, $X$ and $Y$. There is nothing magical about the square root; we can use a cube root or a simple percentage (e.g., 50\%). In the end, it depends on the kind of published data that we are dealing with (e.g., how much noise there is

\footnotetext{
${ }^{7}$ The natural sequence of $[700,923]$ is $[700,701,702,703, \ldots, 922,923]$
} 
in the data). The detection of sequential type is shown in Algorithm 2. For example, in Figure 6-3, the numbers in column "Service number" have the same number of digits, 4, and ranges from 9001 to 9009 (no missing values in the sequence), hence we consider them sequential. If there are two missing numbers, we will still consider this column sequential because the condition is satisfied $(7>\sqrt{9})$.

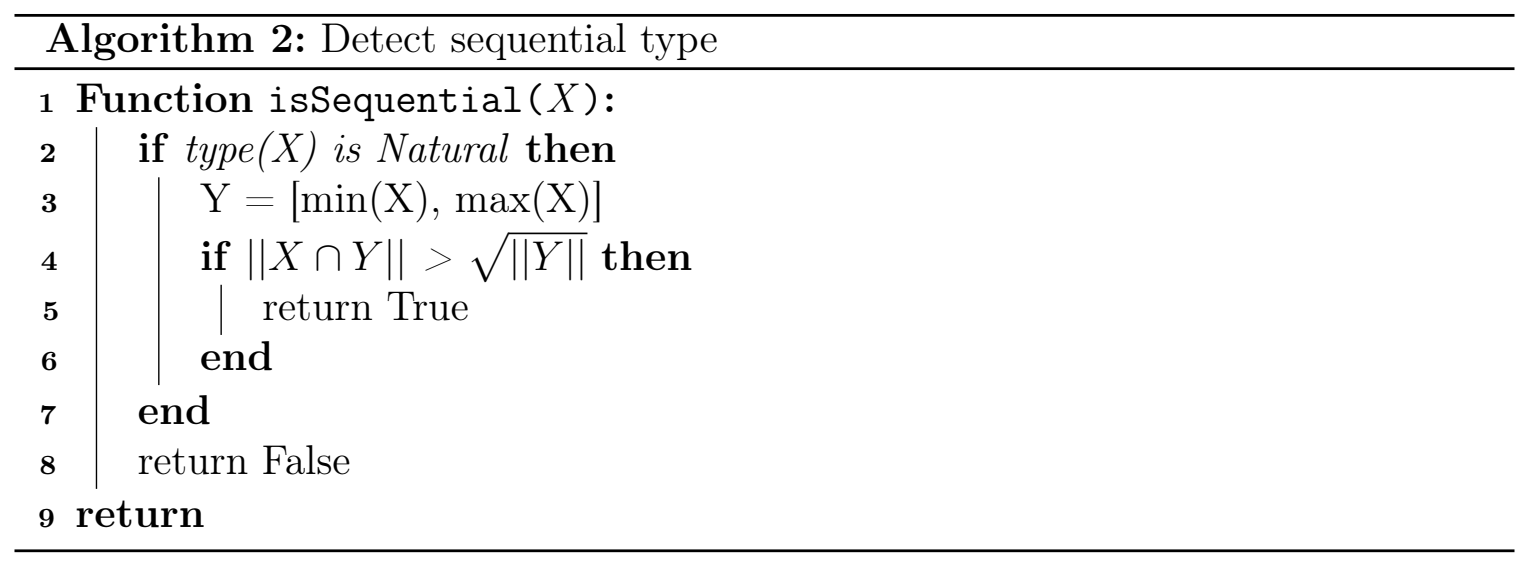

Hierarchical nominal numbers are more difficult to detect. They can be easily confused with another sub-type of nominal and even non-nominal numbers. They tend to include a sequence in their composition. They also have the same number of digits, but this is also the case for the sequential nominal numbers. In order to detect hierarchical data in a column, we first check the number of digits; if it is the same in all the cells, then we consider it to be hierarchical if it is not sequential. This is if the values are unique; having duplicate values is strong evidence of nonhierarchical numbers. This detection method is not perfect, but it is an intuitive way to detect hierarchical nominal numbers. Algorithm 3 shows the method to detect the hierarchical sub-type. In Figure 6-3 in column "Civil ID", all the numbers have the same number of digits, and they fail the sequential test; hence, they will be considered hierarchical.

Categorical nominal numbers have some unique aspects compared to the other nominal numbers. They tend to have a large number of repetitions; the number of categories is an important signal to distinguish it from other categorical data. Another aspect of categorical numbers is that they are usually natural numbers. We consider a list of numbers $X$ as categorical if they are nominal, and the number of unique 


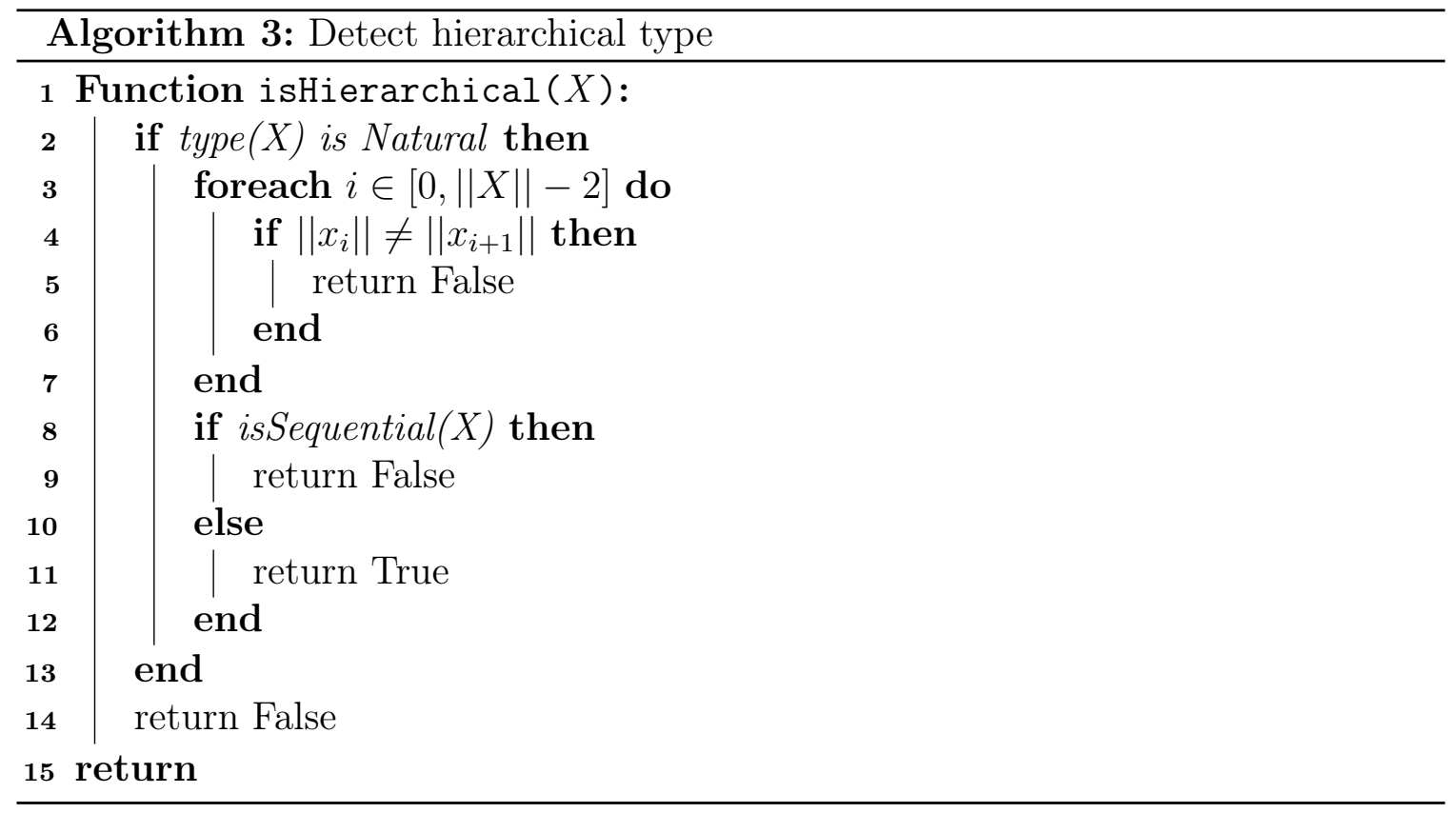

values $U$ is much less $(<<)$ than the total population.

$$
1<\|U\|<\sqrt{\|X\|}
$$

Similar to the sequential sub-type detection, other ways to interpret or execute $<<$ is cube root or log, but they might not be suitable for smaller datasets compared to the square root). We show an example of categorical data in the column "Gender" in Figure 6-3. There are two distinct values $(U=2)$, so $(1<2<\sqrt{9})$ is satisfied, and hence, the column will be considered categorical. We outline the algorithm to detect nominal categorical data in Algorithm 4.

In case there is only one single unique value $(U=1)$, we do not consider that categorical. We ignore that collection as extra knowledge would be needed to understand the meaning of this number.

Random nominal numbers are all of the remaining nominal numbers. However, we have no way of detecting that, which makes sense because it is random. Usually, this kind of numbers is manually removed, as discussed by [47, 52]. 


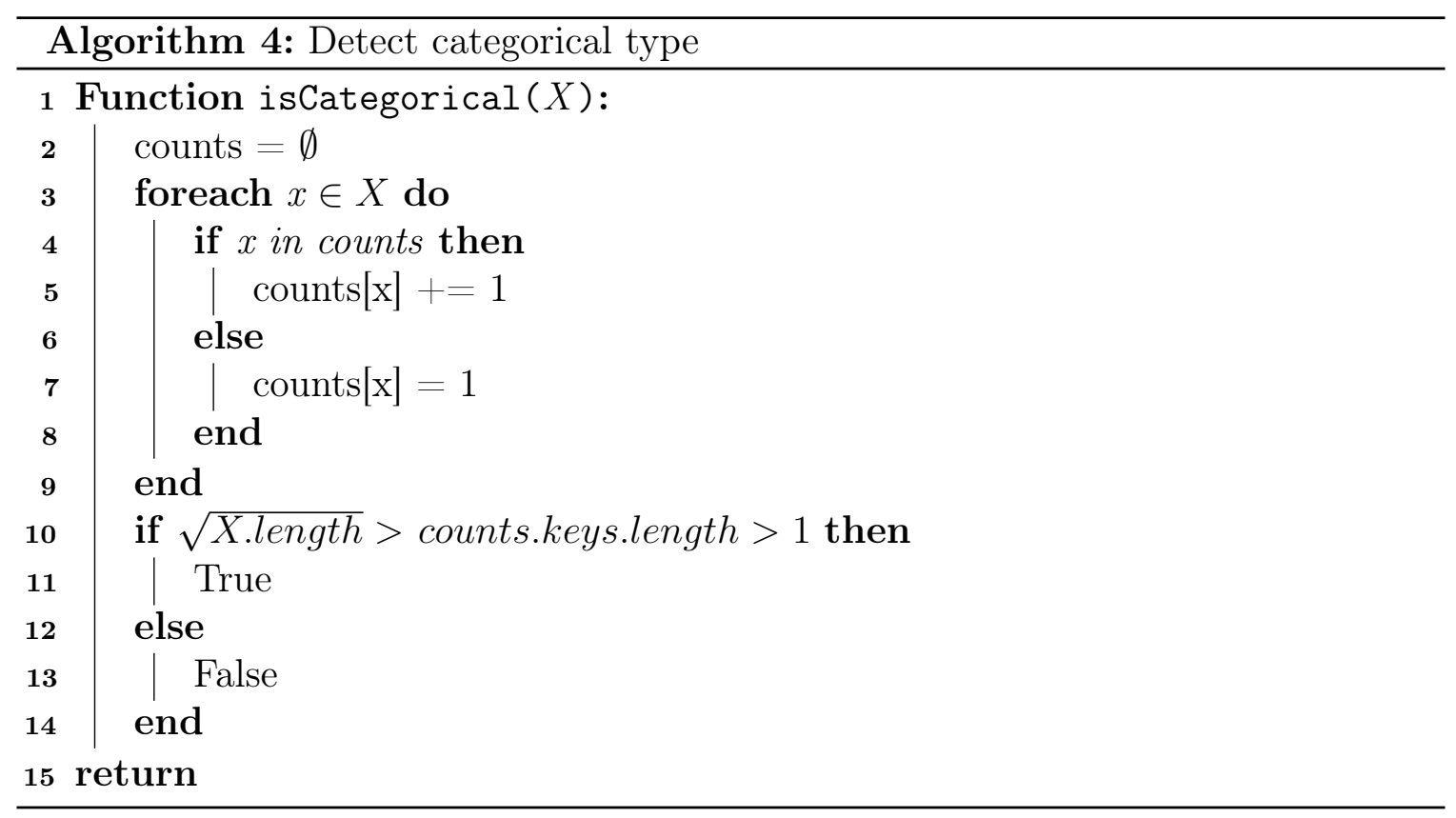

\subsection{Ordinal}

The ordinal scale is one of the easiest scales to detect. Generally, it is just a sequence of natural numbers starting from 1 until $n$ (while $n$ is the number of elements in the sequence). An intuitive way to detect them is to see whether the set of numbers $X$ (what we want to examine) is equal to the list of numbers from 1 until the size of the list. Having a set of negative numbers or floats is a sign that the list of numbers we are examining is not ordinal (Algorithm 5). For example, if we have nine elements, they are ordinals if they range from 1 to 9 (see column "Race rank" in Figure 6-3).

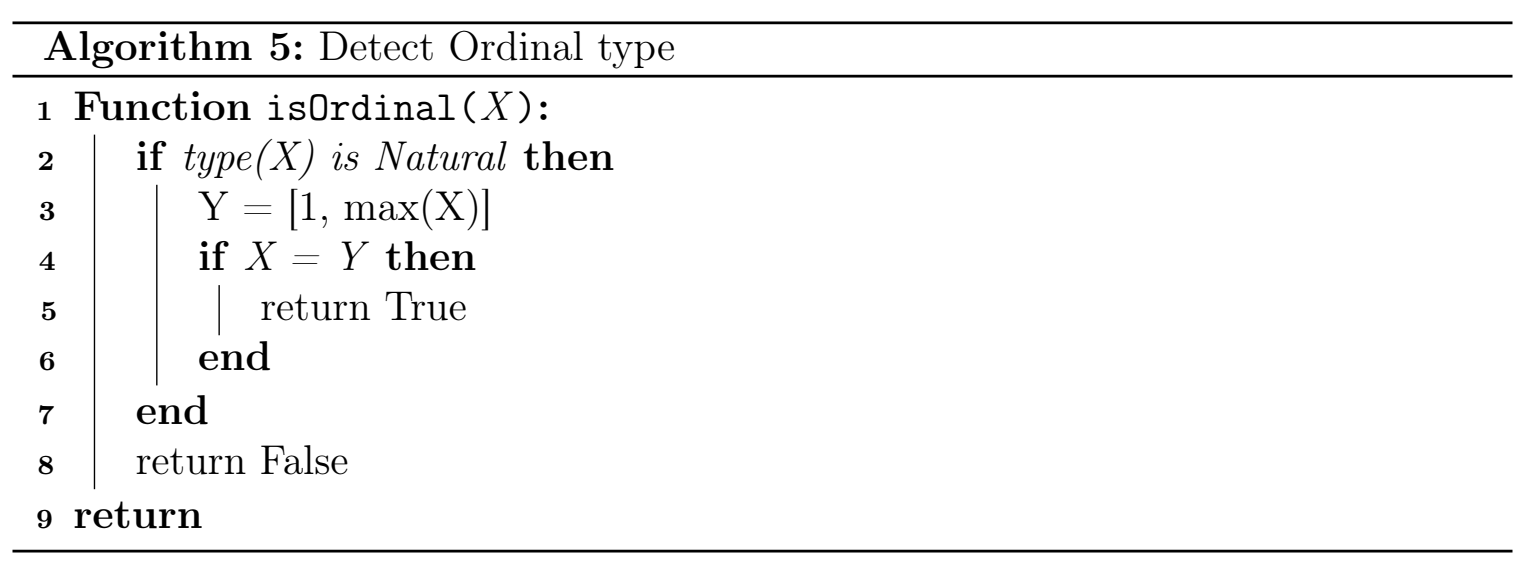




\subsection{Ratio and Interval}

The numbers in the counts sub-type are positive by nature and do not have fractions. One of the main aspects of counts is the way the distances between the numbers increase. Let us say we have a list of numbers of the sub-type counts $X$, if we order the numbers ascendingly,

$$
\forall x \in X: x_{i}<x_{i+1}
$$

the distance between one number and the following one increases rapidly

$$
\begin{array}{r}
\forall x \in X:\left(x_{i+1}-x_{i}\right)<<\left(x_{j+1}-x_{j}\right) \\
j>>i ; j, i \in[0, n-1]
\end{array}
$$

where $n$ is the number of elements in $X^{8}$. To check if the numbers falling under the ratio-interval umbrella are simple counts, we use the following:

$$
\begin{gathered}
1.5 *\left(Q_{3}-Q_{1}\right)+Q_{3} \leq P_{95} \\
\frac{\left(P_{95}-Q_{2}\right)}{Q_{2}} \geq \beta
\end{gathered}
$$

$P_{95}$ refers to the 95 percentile and $Q_{1}, Q_{2}$, and $Q_{3}$ refers to the first, second, and third quartiles, respectively. Formula 6.4 checks whether $P_{95}$ is considered an outlier or not. Following the intuition that the counts sub-type tends to increase a lot at the end (if ordered increasingly). We pick the $P_{95}$ instead of the $P_{100}$ to avoid possible noise or outliers (which can cause it to be falsely positive). Formula (6.5), follows the same intuition that counts tend to have a large increase at the top (large) percentiles. Here we check if it doubles $(\beta=2)$ the numbers in the middle (knows as median or $Q_{2}$ ). Note that in some cases, the optimal value of $\beta$ is greater or less than 2 , but we found it to be a good balance in our preliminaries exploratory tests. If (6.4) and (6.5)

\footnotetext{
${ }^{8}$ This is inspired by the work of Tukey [78] in the analysis of counts data.
} 
are not satisfied, then we consider them to be of the sub-type other (Algorithm 6).

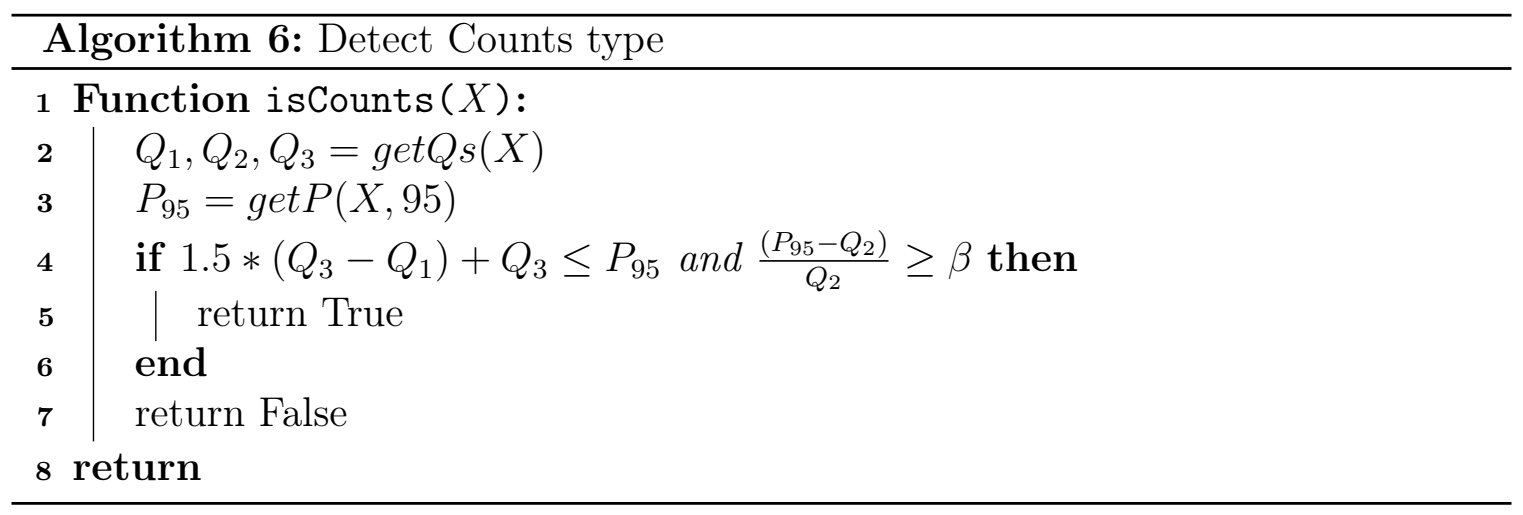

\subsubsection{The Detection Order}

We start by checking whether a column is ordinal because it the most restrictive - it is the only one that checks for exact values. It tests whether the column is composed of natural numbers from 1 until $n$ (where $n$ is the number of elements in the column).

For the second one, it should be one of the sub-types that checks for equal digits (hierarchical, sequential) or categorical. As categorical data contain many duplicates, while sequential and hierarchical do not, we choose to check for categorical first. Then we check for sequential as the hierarchical detection method checks if the numbers have the same number of digits and are not sequential. For the fourth, we check if it is hierarchical.

Last, we check if they are not counts, then they will be considered of the sub-type other as we do not have a specific detection method for it. Note that for the random sub-type (under nominal), we do not have a detection method for it, as mentioned earlier in this section. We show the order of detection in Figure 6-5 and summarize the features in Table 6.4.

\subsubsection{Model}

To build the model, we first extract the numeric values from the knowledge graph. This step is the same as Extraction method 3 in Section 6.1. We use the SPARQL query in Listing 6.4. Then, for each property, we check whether more than $50 \%$ of 


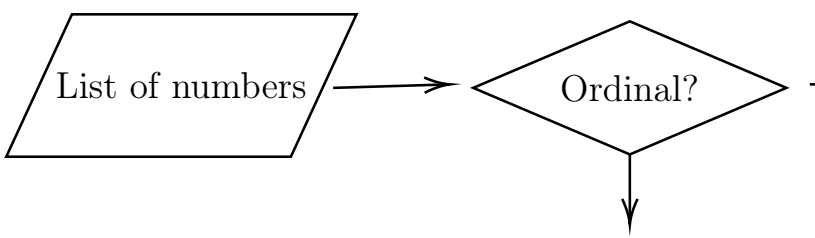

Other

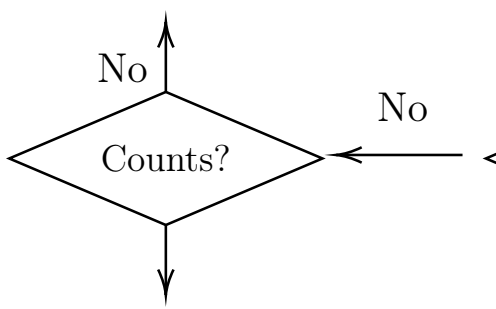

Counts

Ordinal

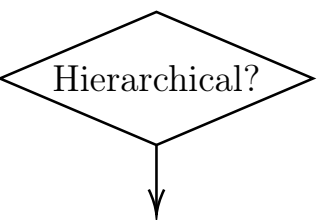

Hierarchical

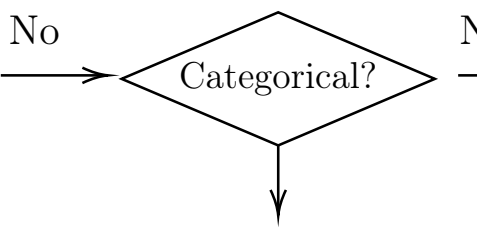

Categorical

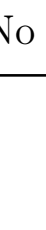

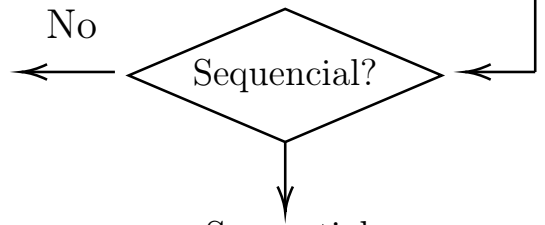

Sequential

Figure 6-5: The detection order

Table 6.4: Typology Detection Order

\begin{tabular}{cccc}
\hline Order & Type & Sub-type & Rules \\
\hline 1 & Ordinal & - & $\in[1, n]$ \\
2 & Nominal & Categorical & few unique numbers \\
3 & Nominal & Sequential & same length ${ }^{*}$ and $\in[\min (X), \max (X)]$ \\
4 & Nominal & Hierarchical & same length $^{*}$ \\
5 & Ratio-Interval & Counts & natural and large increase in values ${ }^{\dagger}$ \\
6 & Ratio-Interval & Other & anything else \\
\hline
\end{tabular}

* All elements have the same number of digits

$\dagger$ Growth similar to quadratic or more

the values (which are known as objects) are numeric. If so, then we consider this property as a numeric property.

After that, we detect the sub-types of the numbers for each property for the corresponding class. The model for each class will be in the format shown in Table 6.5. We show the different model construction methods for each numeric (sub-)type, and we summarize the features for each sub-type in Table 6.6.

\subsection{Nominal}


Table 6.5: Model Features

\begin{tabular}{ccc}
\hline Property URI & Numeric Type/Sub-type & Features \\
\hline$\ldots /$ military-service-number & sequential & $95000, \ldots$ \\
$\ldots /$ height & other & $192.4, \ldots$ \\
$\ldots$ & $\ldots$ & $\ldots$ \\
\hline
\end{tabular}

Table 6.6: Labeling features per sub-type

\begin{tabular}{ccc}
\hline Type & Sub-type & Features \\
\hline nominal & sequential & trimean, tstd \\
nominal & categorical & $\#$ categories $^{*}, \%$ categories $^{\dagger}$ \\
ratio-interval & counts & trimean, tstd \\
ratio-interval & other & trimean, tstd \\
\hline
\end{tabular}

* The number of categories

$\dagger$ The percentages of each category

The features used to build the model depend on whether the numbers are sequential, hierarchical, or categorical. We show how we compute features depending on different kinds of nominal numbers.

For the sequential kind, we use the trimean, which is more resistant to outliers than the mean [78]. We show the formula here:

$$
\text { trimean }=\frac{Q 1+2 * Q 2+Q 3}{4}
$$

We also use the standard deviation with the trimean instead of the mean. We refer to the standard deviation with the trimean as $t s t d$.

For the categorical sub-type, we use the number of unique numbers (the number of categories) followed by the percentages for each category ordered ascendingly.

Matching hierarchical data type with a numeric property from the knowledge graph is complex. If we know the different sections and which digits represent them, 
we would be able to do more ${ }^{9}$, but this is not the case, and hence, we will not be able to annotate them.

For the random sub-type, we ignore it because it is impossible to annotate such kind without extra evidence or elimination of other kinds, as it is random by definition.

\subsection{Ordinal}

This kind of data is common in tabular data. However, we found that it is very limited in knowledge graphs, which makes sense as such ordering usually done with some filtering (e.g., heights in zone A); hence, ordinal numbers are ignored.

\subsection{Ratio and Interval}

We can distinguish the counts sub-type from the other sub-type as they have different aspects that can be exploited to annotate numeric columns.

Raw values of counts as-is are typically hard to understand and analyze [78, 49]. Annotating such data by machines is also difficult as the probability distribution or the numbers (counts) alone does not provide enough evidence to distinguish the data $[78,49]$. Exact match will not be a solution as a single difference in counts data for the same events, but from a different year or reported by a different source could be very close but not exactly the same.

Nonetheless, exploratory data analysis techniques may help here. Although they are generally meant to be used by humans to explore the data, we intend to automate this process. So, we transform the data to help us in understanding it. Exploratory data analysis experts use square $\operatorname{root}(\sqrt{X})$ and $\operatorname{logs}(\log X)$ to analyze the data. Such a transformation of data is referred to as "re-expression" of the data [78, 49]. Logs generally make the results too close while square roots tend to be a good balance between the logs and the raw values [78]; hence, we transform the raw data using the square root. After that, we use the trimean Eq. (6.6) and the trimean-standarddeviation (tstd).

For the sub-type other, we use the trimean [78] Eq. (6.6) and tstd. The use of

\footnotetext{
${ }^{9}$ e.g., treating each section differently or treating each section as a dimension
} 
trimean and tstd instead of the mean and the standard deviation reduces the effects of long-tailed distributions (in the input data and the values of the numeric properties in the knowledge graph).

\subsubsection{Semantic Labeling}

In this section, we discuss the process of semantic labeling for the different types of numeric data, where we treat each type differently to maximize accuracy. We introduce different features specific to each sub-type, as discussed earlier.

For the labeling task, we use the fuzzy c-means clustering technique [10] as we did in our previous approach. We can divide it into two steps: the first one is computing the centers of the clusters; the second step is the classification (to assign semantic labels). Note that in the classical fuzzy c-means, the cluster centers are

computed based on the data points minimizing the total error. In our case, we compute the clusters from the points (features) that we extract from the knowledge graph (Section 6.2.2.3), which are the centers of the clusters. After that, we use the input data of the numeric column and compute the features, which are used to look for the closest cluster. As this is fuzzy clustering, it will belong to multiple clusters with a percentage of the belonging for each.

\subsection{Centroids}

We compute the clusters centers - which are also known as centroids - using the features presented in Section 6.2.2.3. Each numeric property extracted from the knowledge graph has a separate centroid; the value of each centroid is the set of features of the corresponding numeric property.

The reason we compute the centroids that way is that we already know the clusters that correspond to the numeric properties, while in the classical fuzzy c-means, the clusters are not known and need to be discovered by the algorithm.

\subsection{Classification}


Given a collection of numbers as an input (a numeric column), we detect the (sub)type, following the algorithm presented earlier. Then, we retrieve a corresponding model (a model with the same class), and we strip it to include only the numeric properties that match the detected sub-type. Next, we compute the features of the input (numeric column) as in Section 6.2.2.3.

The computed features are then used as new data points. As the features are comma-separated, each value will represent a dimension (see Tables 6.5 and 6.6). For each data point - which are the features computed for a given numeric column - the membership vector is computed as in Eq. (2.2) ${ }^{10}$ with fuzziness $(m=2)$, as suggested by Bezdek et al. [10]. This membership vector indicates the percentage of belonging of a given column to each of the clusters (numeric properties) in the model.

An output of the classification is the membership vector. We order the results in descending order with the corresponding cluster and the membership vector, as in Table 6.1. Note that the sum of the membership values for a given data point should be 1 .

\subsubsection{Experiment Design}

In this section, we aim to answer the research question (Q.3); whether taking into account the typology of numeric columns in a dataset, yields better precision in assigning semantic types to numeric columns than using a general technique (where different types of numbers are treated uniformly).

As we are dealing with different kinds of numbers, we divide the input data for each (sub-)type: sequential, hierarchical, categorical, ordinal, counts, and other. We assign the (sub-)type for each numeric column manually, and then we apply our detection methods to measure their performance. Then, taking into account the knowledge that we have about the (sub-)types of numbers, we apply the labeling methods and report the precision, recall, and F1 scores. We do this for each dataset. The results of the detection are a type (e.g., nominal) and a sub-type (e.g., sequential). For the semantic labeling, the output is a property URI (from the knowledge graph) assigned

\footnotetext{
${ }^{10}$ This equation was introduced by Bezdek et al. [10] to compute the membership.
} 
Table 6.7: Typology Detection and Labeling

\begin{tabular}{cccc}
\hline Type & Sub-type & Detect & Label \\
\hline Nominal & Sequential & yes & yes \\
Nominal & Hierarchical & yes & no \\
Nominal & Categorical & yes & yes \\
Nominal & Random & no & no \\
Ordinal & - & yes & no \\
Ratio-Interval & Counts & yes & yes \\
Ratio-Interval & Other & yes & yes \\
\hline
\end{tabular}

to the (passed) numeric column. The source code of the experiment and the data are published [8] (also available on GitHub ${ }^{11}$ ). It also includes the manual annotations and the (sub-)types for each of the numeric columns ${ }^{12}$.

We summarize the (sub-)types that we are detecting and labeling in Table 6.7 with "yes" for the (sub-)types that we can detect or label and "no" for the ones we do not predict.

We measure the performance of our approach using precision, recall, and F1 scores. We compute the precision by dividing the number of correct predictions by the overall predicted ones Eq. (6.1). For the recall, we divide the number of correctly predicted over the total number of the same type and sub-type Eq. (6.2). The final performance measure is the F1 measure, which is shown in Eq. (6.3). These performance measures are used for the detection and labeling experiments.

We use the T2Dv2 dataset [65]: the only benchmark that we found in the literature that has different types of numeric columns. We use 124 numerical columns from that dataset that we were able to understand and for which we find a corresponding property in DBpedia.

\footnotetext{
${ }^{11}$ https://github.com/oeg-upm/ttla

${ }^{12}$ The manual annotation and assignment of the (sub-)types have been done by the author, and the process took around two working days.
} 
Table 6.8: Typology Detection Scores

\begin{tabular}{ccccc}
\hline Numeric Type & Sub-type & Precision & Recall & F1 \\
\hline Nominal & Sequential & 0.0 & 0.0 & N/A \\
Nominal & Hierarchical & N/A & N/A & N/A \\
Nominal & Categorical & N/A & N/A & N/A \\
Nominal & Random & N/A & N/A & N/A \\
Ordinal & - & 0.8 & 1.0 & 0.889 \\
Ratio-Interval & Count & 0.792 & 0.809 & 0.8 \\
Ratio-Interval & Other & 0.552 & 0.516 & 0.533 \\
\hline
\end{tabular}

\subsubsection{Experiment Results and Discussion}

We first report the scores for the detection of the typology for each of the numeric columns in Table 6.8. For the sequential, we found that there is only one column in the dataset, and we got it wrong. The reason is the noise; the file contains multiple tables with headers located below each other, which does not make the column look like an actual sequence(it looks like a subset of different columns merged together into a single column).

From the benchmark T2Dv2, we did not find any hierarchical or categorical columns hence the $\mathrm{N} / \mathrm{A}$ in the table. For the ordinal, we got high precision and recall applying a simple function. For the counts, we reached a precision score of approximately 0.8 and a similar recall. We inspected the wrongly detected ones, and we see that they do not look like counts. Counts tend to have a high variance as the number increases, which was not the case in a couple of the columns that were wrongly detected. For the last type, it is when the data do not match any of the other sub-types, so there is no specific detection algorithm for it.

This lack of numeric types and sub-types (e.g., Categorical, Hierarchical) and the small number of other sub-types (only one occurrence of the sub-type Sequential) shows the need for a better benchmark. This would help us to evaluate our typology 
Table 6.9: Typology Labeling Scores

\begin{tabular}{|c|c|c|c|c|c|}
\hline $\mathrm{k}$ & Numeric Type & Sub-type & Precision@k & Recall@k & F1@k \\
\hline \multirow{4}{*}{1} & Nominal & Sequential & 1.0 & 1.0 & 1.0 \\
\hline & Ratio-Interval & Count & 0.822 & 0.949 & 0.881 \\
\hline & Ratio-Interval & Other & 0.357 & 0.588 & 0.444 \\
\hline & All & - & 0.649 & 0.842 & 0.733 \\
\hline \multirow{4}{*}{3} & Nominal & Sequential & 1.0 & 1.0 & 1.0 \\
\hline & Ratio-Interval & Count & 0.956 & 0.956 & 0.956 \\
\hline & Ratio-Interval & Other & 0.893 & 0.781 & 0.833 \\
\hline & All & - & 0.932 & 0.885 & 0.908 \\
\hline \multirow{4}{*}{5} & Nominal & Sequential & 1.0 & 1.0 & 1.0 \\
\hline & Ratio-Interval & Count & 1.0 & 0.957 & 0.978 \\
\hline & Ratio-Interval & Other & 0.929 & 0.788 & 0.852 \\
\hline & All & - & 0.973 & 0.889 & 0.929 \\
\hline \multirow{4}{*}{10} & Nominal & Sequential & 1.0 & 1.0 & 1.0 \\
\hline & Ratio-Interval & Count & 1.0 & 0.957 & 0.978 \\
\hline & Ratio-Interval & Other & 1.0 & 0.8 & 0.889 \\
\hline & All & - & 1.0 & 0.892 & 0.943 \\
\hline
\end{tabular}

detection and semantic labeling approaches better.

For the labeling part, we report the precision, recall, and the F1 score. We do that for different values of $k$, taking the top $k$ properties that are the most probable. For $k=1$ (taking into account only the top suggested property), the resulted recall was high $(>0.8)$ while the precision was lower $(>0.7)$. Looking closely at the type with the lowest precision, we found that it is the other sub-type; we were not expecting the precision of it to be low.

We inspected the wrongly labeled properties and found that some are due to being labeled to knowledge graph specific/internal properties (e.g., dbo:wikiPageID) or wrong type detection. For example, the type of areaOfCatchment is wrongly 
detected as of sub-type counts when building the model (while it should have been other). Also, a couple of them wrongly point to properties related to years (e.g., dbp:yearLeader, dbo:eruption Year). We found that years are, again, one of the most difficult to work with, relying only on the values [76, 3]. This could also be improved by having a better type detection, but may not prevent wrongly assigning year-related properties if the used typology does not have a (sub-)type year.

Not only the sub-type other has wrongly labeled properties, but also other types have mislabeled properties. We scrutinized and found that there are properties that are wrong in the knowledge graph. For example, the $d b p$ :iosNumber of a dbo:Currency is labeled as dbp:width; we looked it up and found that there is nothing called the width of a currency. Looking at the values, they look similar to the values of $d b p: i o s N u m b e r$, which makes sense why it was misclassified as dbp:iosNumber.

As the $k$ increases and takes more properties into account, we notice the significant score increase, especially for the precision of the sub-type other; it rises from 0.357 to 0.893 . This means that those correct properties are still in the top suggested ones for the most part.

We have carefully checked the result for recall for the sub-type sequential. This may be misleading because there was only one column with that type and the algorithm got it correctly; so the two possible outcomes were 0 or 1 . We did not report other (sub-)types: hierarchical, categorical, random, and ordinal. This is due to the lack of these types in either the input dataset or the knowledge graph. An exception to that is sub-type random; the reason is that we do not have a way to detect a such (sub-)type. As a result, the sub-type random is reported with the sub-type other (combined).

We compare our approach with our previous work [3] as we did not find any other approach reporting the classification of numeric columns separately for publicly available tabular datasets with numeric columns. We denote our approach in Table 6.10 with TTLA (which stands for Tabular Typology-based LAbeling) and our previous approach with FCM as denoted in the previous section. The scores of our approach (TTLA) for $k=1$ are twice the precision of the previous work (FCM). TTLA contin- 
Table 6.10: Labeling scores of FCM and TTLA approaches applied to T2Dv2

\begin{tabular}{ccccc}
\hline $\mathrm{k}$ & Approach & Precision@k & Recall@k & F1@k \\
\hline \multirow{2}{*}{1} & TTLA & $\mathbf{0 . 6 4 9}$ & 0.842 & 0.733 \\
& FCM & 0.34 & 1.0 & 0.51 \\
\hline \multirow{2}{*}{3} & TTLA & $\mathbf{0 . 9 3 2}$ & 0.885 & 0.908 \\
& FCM & 0.55 & 1.0 & 0.71 \\
\hline 5 & TTLA & $\mathbf{0 . 9 7 3}$ & 0.889 & 0.929 \\
& FCM & 0.83 & 1.0 & 0.91 \\
\hline \multirow{2}{*}{10} & TTLA & $\mathbf{1 . 0}$ & 0.892 & 0.943 \\
& FCM & 0.91 & 1.0 & 0.95 \\
\hline
\end{tabular}

ues to out-perform FCM as we increase $k$. We can see a clear significant improvement of precision.

We show the typology found in the dataset in Table 6.11. We can see that the most prominent lies under the ratio-interval type. The count type takes close to 0.4 of the total number of numerical columns. This has been reported in our previous paper [3] when talking about long-tailed distributions, which often caused by the data being condensed in one of the sides far from the mean. This was the primary cause of incorrect labeling in that work. We also notice here that we have a type called year, which is not mentioned in the background nor in the typology that we adopt. This is due to its unique properties. The type year is confusing as it can be thought of as a simple count from the birth of Jesus until a given moment. This could also be thought of as a nominal since it is often used to tag and name things produced, created, or occurred in a year without any regard to the counting aspect. Since it can be compared, some might argue that it is not nominal (which does not encompass the greater or less than operators [72]). Due to that, we ignored it in our experiments. 
Table 6.11: Typology in T2Dv2 dataset

\begin{tabular}{ccc}
\hline Numeric Type & Sub-type & Percentage \\
\hline Nominal & Sequential & 0.008 \\
Nominal & Hierarchical & 0.0 \\
Nominal & Categorical & 0.0 \\
Nominal & Random & 0.048 \\
Nominal & combined & 0.056 \\
Ordinal & - & 0.04 \\
Ratio-Interval & Count & 0.387 \\
Ratio-Interval & Other & 0.234 \\
Ratio-Interval & combined & 0.621 \\
Year & - & 0.282 \\
\hline
\end{tabular}




\section{Chapter 7}

\section{Conclusion and Future Work}

A vast amount of tabular data are published without any annotations that may make them easier to reuse. Such data are published in different formats such as CSV (comma separated values) and Excel Binary File Format (.xls). Understanding the content of such tables is crucial for data integration, exploitation and reuse. Manual processing, understanding, and linking of large collections of tables is error prone and does not scale. Several approaches have been already introduced in the literature to link such data together, but not all of them use semantic-based annotations [14, 84]. Several approaches add semantics to increase the exploitability of such data $[61,85$, $64,52,26,86,63]$. We explored the literature and observed several shortcomings, such as the reliance on search engines, the dependence on fixed sources of knowledge, and the need for manual intervention at some steps. By studying several of the existing techniques, we developed a few ideas of how to overcome such drawbacks. We formulated three research questions, exploring our initial ideas to overcome the drawbacks we observed and then hypothesized that it is possible to semantically label the majority of subject and numeric columns without suffering from such limitations.

We answer Q.1 - whether we can annotate the majority of subject columns automatically using knowledge graphs as the only source of knowledge, without considering the context in which the data appear, and without using other external sources of information and knowledge graph profiling beforehand - in Chapter 5. We developed an algorithm to add potential classes to a given subject column using a given 
knowledge graph. The algorithm first performs a simple entity linking by querying the knowledge graph for entities that share the same name/label as the cell value. After the cells in the subject columns are linked to possible entities, the types of these candidate entities are scored. The proposed scoring is based on two intuitions: specific classes are more valuable than generic classes, and generic types are more prominent than specific classes. However, the process of entity linking can easily link to incorrect entities that share the same name/label. This would introduce invalid classes as candidates. So we should also take into account that rare specific classes are not prominent in comparison with the correct classes. We formalized these intuitions into scoring functions to score each candidate class. The class with the highest score is then picked as the class of the subject column. Table 5.3 shows the performance of our semantic labeling approach. We tested the algorithm with the well-known T2Dv2 dataset and reached 0.91 of precision, 0.97 of recall, and 0.94 of the F1 score. The approach only takes into account the values in the subject columns, without using any context, without relying on other external sources of information, without profiling the knowledge graph beforehand, and without manual intervention. Hence, with our approach, we can label the majority of subject columns, and Q.1 is answered.

To answer Q.2, whether we can automatically match most of the numeric columns in tabular data to their corresponding properties in knowledge graphs in the absence of context and without external sources of information without performing entity linking given the type of the subject, we perform the experiment in Chapter 6, Section 6.1. The idea is to pick the most similar numeric property (of a knowledge graph) to a given list of numbers (a numeric column) from a dataset. However, often the numbers in datasets might include much fewer numbers than properties in knowledge graphs. They also might differ in the accuracy (e.g., number of fractional digits). We also notice a big noise in the data distribution of numeric properties, which was erroneous. We developed an algorithm based on the fuzzy c-means clustering technique taking into account these observations. We manually annotated the numeric columns in the T2Dv2 datasets and tested our algorithm against the manually annotated numeric columns. The algorithm reached a precision of 0.34 , a recall of 1.0, and an F1 
score of 0.51 (Table 6.3). So, we were not able to answer this research question adequately. Looking closely at the mislabeled columns, we noticed a pattern. Different kinds/types of numeric values have different characteristics. We adopted the numeric kinds proposed by Stevens [72] and extended it to fit the need in semantic labeling of numeric data. However, we also need a way to identify the numeric kind/type of a collection of numeric values automatically. We adopted techniques from exploratory data analysis [78, 49] to automatically detect the numeric kinds/types. We tested our extended version of the approach, taking into account the typology (Section 6.2). Using the typology-based semantic labeling, we achieved 0.640 of precision, 0.842 of recall, and 0.733 of the F1 score (Table 6.10). This answers Q.2 and Q.3 - taking the numeric typology into account improves the semantic labeling scores of numeric columns.

As shown in our experiments, our approach was able to automatically label the majority of subject and numeric columns in the only available (and easy to use) benchmark of annotated tables. Based on that, our hypothesis - that the majority of subject and numeric columns in tabular data on the Web can be labeled automatically using knowledge graphs as the only external source of information - is proved to be true on the available tabular data benchmark, T2Dv2.

Furthermore, our approach managed to produce competitive results compared to the state-of-the-art approaches that rely on more information. That is, the approach that we propose does not take into account the headers of the tables, the URL of the tables, the captions, or the text surrounding the tables. So this means that our approach may be used in situations where there is no extra metadata about the table (e.g., when a user is directly editing an Excel file and may want to annotate the file semantically). Also, it does not rely on linguistic resources or wikis. This means that in the case of very specialized domains where such resources may not be available, our approaches would still work.

To present the applicability of our approaches, we are offering them as services, so that they can be used by users without installing any software. We also built an editor to assign semantic annotations to user uploaded tables. It suggests the most 
probable classes and properties for the user table, providing them the possibility to pick one of the suggestions or use the autocomplete feature to assign the semantic classes and properties. It also permits the user to export the labelings as RML or R2RML mappings, so as to provide access to the datasets as a unified global view of the data. This also permits the user to query the underlying datasets with SPARQL.

Nonetheless, an argument may arise that current benchmarks are not representative of the data published on the Web. As far as we know, there is no published work about this matter. Also, from our understanding, having an annotated subset that is representative of the Web is not yet done, and we do not think it will be feasible to obtain at present, but this is our speculation, based on the difficulty of this challenge.

In spite of all the great efforts to interpret tables using agreed-upon vocabularies, there is a need for more extensive benchmarks that are more representative of the tabular data published on the Web. We detected the absence of multiple numeric types in the published benchmarks (e.g., hierarchical). Also, the domains covered are very limited in comparison to the tabular data on the Web. However, the process of creating a benchmark is challenging. It is time-consuming and requires a lot of effort. To avoid bias and human error, often, the process involves multiple human annotators for each annotation. We are exploring ways to create a bigger benchmark with multiple annotators. The idea is to build the benchmark in a semi-automatic manner to ease the process of the annotators while making use of the already annotated data. The application would pick a small subset of the data to be annotated and provide the user with multiple choice options to pick. The data are then aggregated, and disagreement of annotations are analyzed. The last step would be to export the annotations of the data as mappings (e.g., RML, R2RML, CSV on the Web).

We are also exploring ways for further improvements in our approaches. We are seeking for more annotated numeric data to improve the detection algorithms. We are also working to address tables with subjects split into multiple columns (e.g., first name and last name) or tables with no subject columns (which is common in weather data). We are also looking into including the use of advanced entity linking approaches as part of the process, as that has been done in approaches like $[23,79$, 
$80,61,84,56,26,63]$ so we can facilitate even more the process of creating semantic annotations of any table. Finally, we would like to explore the idea of using multiple knowledge graphs and let the system decide which one is the most preferred for a given data source, or use both to improve the overall score. One option can be performing our approach on each of the knowledge graphs separately and then select the knowledge graph that yields the highest score. Another approach would be to introduce an abstraction layer on top and query several knowledge graphs as if they were a single one. Several challenges may arise in both options, and we plan to explore them in the future. 


\section{Bibliography}

[1] IBM FORTRAN Program Products for OS and the CMS Component of VM/370 General Information.

[2] Ahmad Alobaid. Tada-gam, November 2019.

[3] Ahmad Alobaid and Oscar Corcho. Fuzzy semantic labeling of semi-structured numerical datasets. In European Knowledge Acquisition Workshop, pages 19-33. Springer, 2018.

[4] Ahmad Alobaid and Oscar Corcho. Olympic games 2020, September 2018.

[5] Ahmad Alobaid and Oscar Corcho. Tada-entity, 2018.

[6] Ahmad Alobaid and Oscar Corcho. Tada-numcol, September 2018.

[7] Ahmad Alobaid and Oscar Corcho. Knowledge-graph-based semantic labeling: Balancing coverage and specificity. Semantic Web, 2020. Under review.

[8] Ahmad Alobaid, Emilia Kacprzak, and Oscar Corcho. Ttla source code, 2019.

[9] Ahmad Alobaid, Emilia Kacprzak, and Oscar Corcho. Typology-based semantic labeling of numeric tabular data. Semantic Web, 2020. accepted.

[10] James C Bezdek, Robert Ehrlich, and William Full. Fcm: The fuzzy c-means clustering algorithm. Computers \& Geosciences, 10(2-3):191-203, 1984.

[11] Richard W Bolling, Louis P Tychonievich, Geoffrey E Margrave, David F Shannon, and Eric S Rustici. Expert system with knowledge base having term definition hierarchy, October 30 1990. US Patent 4,967,368.

[12] Michael L Brodie and John Mylopoulos. Knowledge bases vs databases. In On Knowledge Base Management Systems, pages 83-86. Springer, 1986.

[13] Cary D Butler, David R Richards, Robert M Wallace, Norman L Jones, and Russell Jones. extensible model data format (xmdf). Technical report, ENGINEER RESEARCH AND DEVELOPMENT CENTER VICKSBURG MS INFORMATION TECHNOLOGY LAB, 2007. 
[14] Michael J Cafarella, Alon Halevy, Daisy Zhe Wang, Eugene Wu, and Yang Zhang. Webtables: exploring the power of tables on the web. Proceedings of the VLDB Endowment, 1(1):538-549, 2008.

[15] Michael J Cafarella, Alon Y Halevy, Yang Zhang, Daisy Zhe Wang, and Eugene Wu. Uncovering the relational web. In WebDB, 2008.

[16] David Chaves-Fraga, Freddy Priyatna, Idafen Santana-Pérez, and Óscar Corcho. Virtual statistics knowledge graph generation from csv files. In ISWC (Best Workshop Papers), pages 235-244, 2018.

[17] Nicholas R Chrisman. Rethinking levels of measurement for cartography. Cartography and Geographic Information Systems, 25(4):231-242, 1998.

[18] Douglas Crockford. The JavaScript Object Notation (JSON) Data Interchange Format. RFC 7159, March 2014.

[19] Jeffrey Dean and Sanjay Ghemawat. Mapreduce: Simplified data processing on large clusters. 2004.

[20] Jeffrey Dean and Sanjay Ghemawat. Mapreduce: simplified data processing on large clusters. Communications of the ACM, 51(1):107-113, 2008.

[21] Dong Deng, Yu Jiang, Guoliang Li, Jian Li, and Cong Yu. Scalable column concept determination for web tables using large knowledge bases. Proceedings of the VLDB Endowment, 6(13):1606-1617, 2013.

[22] Anastasia Dimou, Miel Vander Sande, Pieter Colpaert, Ruben Verborgh, Erik Mannens, and Rik Van de Walle. Rml: a generic language for integrated rdf mappings of heterogeneous data. 2014.

[23] Mark Dredze, Paul McNamee, Delip Rao, Adam Gerber, and Tim Finin. Entity disambiguation for knowledge base population. In Proceedings of the 23rd International Conference on Computational Linguistics, pages 277-285. Association for Computational Linguistics, 2010.

[24] Patrick Durusau and Michael Brauer. Open Document Format for Office Applications (OpenDocument) v1.0 (Second Edition). Technical report, July 2006.

[25] Lisa Ehrlinger and Wolfram Wöß. Towards a definition of knowledge graphs. SEMANTiCS (Posters, Demos, SuCCESS), 48, 2016.

[26] Ivan Ermilov and Axel-Cyrille Ngonga Ngomo. Taipan: automatic property mapping for tabular data. In European Knowledge Acquisition Workshop, pages 163-179. Springer, 2016.

[27] Michael Färber, Frederic Bartscherer, Carsten Menne, and Achim Rettinger. Linked data quality of dbpedia, freebase, opencyc, wikidata, and yago. Semantic Web, 9(1):77-129, 2018. 
[28] Christina Feilmayr and Wolfram Wöß. An analysis of ontologies and their success factors for application to business. Data \& Knowledge Engineering, 101:1-23, 2016.

[29] Jenny Rose Finkel, Trond Grenager, and Christopher Manning. Incorporating non-local information into information extraction systems by gibbs sampling. In Proceedings of the 43rd annual meeting on association for computational linguistics, pages 363-370. Association for Computational Linguistics, 2005.

[30] Peter Flach. Machine learning: the art and science of algorithms that make sense of data. Cambridge University Press, 2012.

[31] Aman Goel, Craig A Knoblock, and Kristina Lerman. Exploiting structure within data for accurate labeling using conditional random fields. In Proceedings on the International Conference on Artificial Intelligence (ICAI), page 1. The Steering Committee of The World Congress in Computer Science, Computer Engineering and Applied Computing (WorldComp), 2012.

[32] Burton Grad. The creation and the demise of visicalc. IEEE Annals of the History of Computing, 29(3):20-31, 2007.

[33] FITS Working Group et al. Definition of the flexible image transport system (fits). FITS Standard Version, 3, 2008.

[34] Thomas R Gruber. A translation approach to portable ontology specifications. Knowledge acquisition, 5(2):199-220, 1993.

[35] Claudio Gutierrez and Juan F. Sequeda. A brief history of knowledge graph's main ideas: A tutorial. October 2019.

[36] Oktie Hassanzadeh, Michael J Ward, Mariano Rodriguez-Muro, and Kavitha Srinivas. Understanding a large corpus of web tables through matching with knowledge bases: an empirical study. In $O M$, pages 25-34, 2015.

[37] Aidan Hogan, Eva Blomqvist, Michael Cochez, Claudia d'Amato, Gerard de Melo, Claudio Gutierrez, José Emilio Labra Gayo, Sabrina Kirrane, Sebastian Neumaier, Axel Polleres, Roberto Navigli, Axel-Cyrille Ngonga Ngomo, Sabbir M. Rashid, Anisa Rula, Lukas Schmelzeisen, Juan Sequeda, Steffen Staab, and Antoine Zimmermann. Knowledge graphs, 2020.

[38] Aidan Hogan and Dan Brickley. (re)defining knowledge graphs. Knowledge Graphs: New Directions for Knowledge Representation on the Semantic Web, page $74,2018$.

[39] Anil K Jain, M Narasimha Murty, and Patrick J Flynn. Data clustering: a review. ACM computing surveys (CSUR), 31(3):264-323, 1999. 
[40] Emilia Kacprzak, José M Giménez-García, Alessandro Piscopo, Laura Koesten, Luis-Daniel Ibáñez, Jeni Tennison, and Elena Simperl. Making sense of numerical data-semantic labelling of web tables. In European Knowledge Acquisition Workshop, pages 163-178. Springer, 2018.

[41] Craig A Knoblock, Pedro Szekely, José Luis Ambite, Aman Goel, Shubham Gupta, Kristina Lerman, Maria Muslea, Mohsen Taheriyan, and Parag Mallick. Semi-automatically mapping structured sources into the semantic web. In Extended Semantic Web Conference, pages 375-390. Springer, 2012.

[42] Tauno Knuuttila et al. Nuclear magnetism and superconductivity in rhodium. Helsinki University of Technology, 2000.

[43] Sandeep Koranne. Hierarchical data format 5: Hdf5. In Handbook of Open Source Tools, pages 191-200. Springer, 2011.

[44] David Lane, Joan Lu, Camille Peres, Emily Zitek, et al. Online statistics: An interactive multimedia course of study. Retrieved January, 29:2009, 2008.

[45] Girija Limaye, Sunita Sarawagi, and Soumen Chakrabarti. Annotating and searching web tables using entities, types and relationships. Proceedings of the VLDB Endowment, 3(1-2):1338-1347, 2010.

[46] Anders Steen Lunde. The person-number systems of sweden, norway, denmark, and israel. 1980.

[47] Albert Merono Penuela. Refining statistical data on the web. Ph.D. thesis, Vrije Universiteit Amsterdam, 2016.

[48] Nandana Mihindukulasooriya, Freddy Priyatna, Oscar Corcho, Raúl GarcíaCastro, and Miguel Esteban-Gutiérrez. morph-ldp: an r2rml-based linked data platform implementation. In European Semantic Web Conference, pages 418423. Springer, 2014.

[49] Frederick Mosteller and John Wilder Tukey. Data analysis and regression: a second course in statistics. Addison-Wesley Series in Behavioral Science: Quantitative Methods, 1977.

[50] Varish Mulwad, Tim Finin, and Anupam Joshi. Semantic message passing for generating linked data from tables. In International Semantic Web Conference, pages 363-378. Springer, 2013.

[51] John Mylopoulos, Vinay Chaudhri, Dimitris Plexousakis, Adel Shrufi, and Thodoros Topologlou. Building knowledge base management systems. The VLDB Journal - The International Journal on Very Large Data Bases, 5(4):238$263,1996$. 
[52] Sebastian Neumaier, Jürgen Umbrich, Josiane Xavier Parreira, and Axel Polleres. Multi-level semantic labelling of numerical values. In International Semantic Web Conference, pages 428-445. Springer, 2016.

[53] Nurzhan Nurseitov, Michael Paulson, Randall Reynolds, and Clemente Izurieta. Comparison of json and $\mathrm{xml}$ data interchange formats: a case study. Caine, 9:157-162, 2009.

[54] Yaser Oulabi and Christian Bizer. Extending cross-domain knowledge bases with long tail entities using web table data. 2019.

[55] Heiko Paulheim. Knowledge graph refinement: A survey of approaches and evaluation methods. Semantic web, 8(3):489-508, 2017.

[56] Minh Pham, Suresh Alse, Craig A Knoblock, and Pedro Szekely. Semantic labeling: a domain-independent approach. In International Semantic Web Conference, pages 446-462. Springer, 2016.

[57] Antonella Poggi, Domenico Lembo, Diego Calvanese, Giuseppe De Giacomo, Maurizio Lenzerini, and Riccardo Rosati. Linking data to ontologies. In Journal on data semantics $X$, pages 133-173. Springer, 2008.

[58] Daniel J Power. A history of microcomputer spreadsheets. Communications of the Association for Information Systems, 4(1):9, 2000.

[59] Daniel J Power. A brief history of spreadsheets. DSSResources. COM, World Wide Web, http://dssresources. com/history/sshistory. html, version, 3:08-30, 2004.

[60] Freddy Priyatna, Oscar Corcho, and Juan Sequeda. Formalisation and experiences of r2rml-based sparql to sql query translation using morph. In Proceedings of the 23rd international conference on World wide web, pages 479-490. ACM, 2014.

[61] Gianluca Quercini and Chantal Reynaud. Entity discovery and annotation in tables. In Proceedings of the 16th International Conference on Extending Database Technology, pages 693-704. ACM, 2013.

[62] SK Ramnandan, Amol Mittal, Craig A Knoblock, and Pedro Szekely. Assigning semantic labels to data sources. In European Semantic Web Conference, pages 403-417. Springer, 2015.

[63] Dominique Ritze and Christian Bizer. Matching web tables to dbpedia-a feature utility study. context, 42(41):19, 2017.

[64] Dominique Ritze, Oliver Lehmberg, and Christian Bizer. Matching html tables to dbpedia. In Proceedings of the 5th International Conference on Web Intelligence, Mining and Semantics, page 10. ACM, 2015. 
[65] Dominique Ritze, Oliver Lehmberg, and Christian Bizer. T2Dv2 Gold Standard for Matching Web Tables to DBpedia. http://webdatacommons.org/ webtables/goldstandardV2.html, 2015. [Online; accessed 16-August-2018].

[66] Dominique Ritze, Oliver Lehmberg, Yaser Oulabi, and Christian Bizer. Profiling the potential of web tables for augmenting cross-domain knowledge bases. In Proceedings of the 25th International Conference on World Wide Web, pages 251-261. International World Wide Web Conferences Steering Committee, 2016.

[67] Mayra Z Rodriguez, Cesar H Comin, Dalcimar Casanova, Odemir M Bruno, Diego R Amancio, Luciano da F Costa, and Francisco A Rodrigues. Clustering algorithms: A comparative approach. PloS one, 14(1):e0210236, 2019.

[68] Y. Shafranovich. Common Format and MIME Type for Comma-Separated Values (CSV) Files. RFC 4180, October 2005.

[69] Wei Shen, Jianyong Wang, and Jiawei Han. Entity linking with a knowledge base: Issues, techniques, and solutions. IEEE Transactions on Knowledge and Data Engineering, 27(2):443-460, 2015.

[70] Amit Singhal. Introducing the knowledge graph: things, not strings. Official google blog, 5, 2012.

[71] John F Sowa. Semantic networks. John_Florian_Sowa isi [2012-04-20 16: 51]> Author [2012-04-20 16: 51], 2012.

[72] Stanley Smith Stevens et al. On the theory of scales of measurement. American Association for the Advancement of Science, Science, New Series, Vol. 103, No. $2684,1946$.

[73] Rudi Studer, V Richard Benjamins, and Dieter Fensel. Knowledge engineering: principles and methods. Data E3 knowledge engineering, 25(1-2):161-197, 1998.

[74] Fabian M Suchanek, Serge Abiteboul, and Pierre Senellart. Paris: Probabilistic alignment of relations, instances, and schema. Proceedings of the VLDB Endowment, 5(3):157-168, 2011.

[75] Zareen Syed, Tim Finin, Varish Mulwad, and Anupam Joshi. Exploiting a web of semantic data for interpreting tables. In Proceedings of the Second Web Science Conference, volume 5, 2010.

[76] Mohsen Taheriyan, Craig A Knoblock, Pedro Szekely, and José Luis Ambite. Learning the semantics of structured data sources. Web Semantics: Science, Services and Agents on the World Wide Web, 37:152-169, 2016.

[77] Alberto Tonon, Michele Catasta, Gianluca Demartini, Philippe Cudré-Mauroux, and Karl Aberer. Trank: Ranking entity types using the web of data. In International Semantic Web Conference, pages 640-656. Springer, 2013. 
[78] John W Tukey. Exploratory data analysis. 1977. Massachusetts: Addison-Wesley, 1976.

[79] Ricardo Usbeck, Axel-Cyrille Ngonga Ngomo, Michael Röder, Daniel Gerber, Sandro Athaide Coelho, Sören Auer, and Andreas Both. Agdistis-graph-based disambiguation of named entities using linked data. In International semantic web conference, pages 457-471. Springer, 2014.

[80] Petros Venetis, Alon Halevy, Jayant Madhavan, Marius Paşca, Warren Shen, Fei Wu, Gengxin Miao, and Chung Wu. Recovering semantics of tables on the web. Proceedings of the VLDB Endowment, 4(9):528-538, 2011.

[81] Gio Wiederhold. Knowledge and database management. IEEE Software, (1):6373, 1984.

[82] Gongqing $\mathrm{Wu}$, Ying He, and Xuegang Hu. Entity linking: An issue to extract corresponding entity with knowledge base. IEEE Access, 6:6220-6231, 2018.

[83] Lotfi A Zadeh. Fuzzy sets, information and control. vol, 8:338-353, 1965.

[84] Meihui Zhang and Kaushik Chakrabarti. Infogather+: Semantic matching and annotation of numeric and time-varying attributes in web tables. In Proceedings of the 2013 ACM SIGMOD International Conference on Management of Data, pages 145-156. ACM, 2013.

[85] Ziqi Zhang. Towards efficient and effective semantic table interpretation. In International Semantic Web Conference, pages 487-502. Springer, 2014.

[86] Ziqi Zhang. Effective and efficient semantic table interpretation using tableminer+. Semantic Web, 8(6):921-957, 2017. 